\title{
Ultrasound cleaning of microfilters
}

\section{Hald, Jens; Bjørnø, Irina; Jensen, Leif Bjørnø}

\section{Published in:}

Acoustical Society of America. Journal

Link to article, DOI:

10.1121/1.426158

Publication date:

1999

\section{Document Version}

Publisher's PDF, also known as Version of record

Link back to DTU Orbit

Citation (APA):

Hald, J., Bjørnø, I., \& Jensen, L. B. (1999). Ultrasound cleaning of microfilters. Acoustical Society of America. Journal, 105(2), 1292-1292. https://doi.org/10.1121/1.426158

\section{General rights}

Copyright and moral rights for the publications made accessible in the public portal are retained by the authors and/or other copyright owners and it is a condition of accessing publications that users recognise and abide by the legal requirements associated with these rights.

- Users may download and print one copy of any publication from the public portal for the purpose of private study or research.

- You may not further distribute the material or use it for any profit-making activity or commercial gain

- You may freely distribute the URL identifying the publication in the public portal

If you believe that this document breaches copyright please contact us providing details, and we will remove access to the work immediately and investigate your claim 
Posters from various technical sessions remain on display in the Poster Gallery.

Posters from sessions which contain both lecture and poster presentations will be attended by the authors as listed below.

\begin{tabular}{|c|c|c|}
\hline \multicolumn{3}{|r|}{ 2:00-4:00 } \\
\hline $3 \mathrm{pNSb} 12$ & Murphy, William J. & Four protectors in search of a rating system \\
\hline $3 \mathrm{pNSb} 13$ & Zannin, Paulo H. T. & Effects of cup, cushion, headband force, and foam lining on the attenuation of earmuffs \\
\hline 5 pNSa10 & Lelong, Joel & Effect of the acceleration on vehicle noise emission \\
\hline \multicolumn{3}{|r|}{$4: 40-6: 20$} \\
\hline $4 \mathrm{pNSd} 8$ & Baumann, Uwe & $\begin{array}{l}\text { Characteristics of MRI acoustic notice in functional and high resolution imaging of the auditory path- } \\
\text { ways }\end{array}$ \\
\hline $4 \mathrm{pNSd} 9$ & Bezvesilna, Olena N. & Adaptive decision method for acoustical turbomachinery diagnostics \\
\hline $4 \mathrm{pNSd} 10$ & Engel, Zbigniew & Investigations of sound insulation of partitions used for integrated enclosures \\
\hline \multicolumn{3}{|r|}{$5: 20-6: 20$} \\
\hline 4 pPAb10 & Poggi, Sylvain & Laser Doppler anemometry applied to airborne acoustics: Influence of the signal processing parameters \\
\hline 4pPAb11 & Urbanczyk, Marian & Acoustoelectric effect in gas sensor of the SAW type \\
\hline 4pPAb12 & Opilski, Aleksander & Optional SAW sensor for low nitrogen dioxide concentration \\
\hline
\end{tabular}

Also the following poster sessions are scheduled:

Poster Session $4 \mathrm{pPPb}$

THURSDAY AFTERNOON, 18 MARCH 1999

ROOM H3010, 12:55 TO 5:20 P.M.

\title{
Session 4pAA \\ Architectural Acoustics: Building Acoustics I: Airborne and Impact Sound Transmission and Classroom Acoustics
}

\author{
Sten Ljunggren, Cochair \\ Department of Building Technology, KTH, Brinellvägen 34, S-100 44 Stockholm, Sweden \\ Alfred C. C. Warnock, Cochair \\ National Research Council, Building M39, Montreal Road, Ottawa, Ontario K1A OR6, Canada \\ Chair's Introduction-12:55 \\ Invited Paper \\ 1:00
}

4pAA1. The influence of identical room dimensions on the sound insulation at low frequencies. Wolfgang Kropp and Tor Kihlman (Dept. of Appl. Acoust., Chalmers Univ. of Technol., SE-41296 Gothenburg, Sweden, wk@ta.chalmers.se)

\begin{abstract}
At low frequencies the sound insulation between rooms is only valid for the specific case under consideration as shown in works by different authors. The expression "low frequencies" implies a frequency range where the rooms' dimensions are comparable with the wavelength of sound in the rooms (i.e., normally below about $250 \mathrm{~Hz}$ for normal rooms). Both theoretical studies as well as experimental studies in the laboratory show that there is a substantial decrease of the sound insulation when the dimensions of the sending and receiving rooms coincide. The question arises if this decrease of the sound insulation can also be observed for the sound insulation of partitions in the field. The results are often biased by other effects such as flanking transmission or smaller differences in the fabrication of the partitions. By means of statistical studies field measurements are analyzed to show the influence of the room dimensions on the sound insulation in the field. To avoid the deterioration of the sound insulation, changes in the dimensions would be required which do not allow for symmetrical building designs as it is often preferred in practice.
\end{abstract}

\section{Contributed Papers}

$1: 20$

4pAA2. Sound transmission loss of double-glazed window panes-an empirical approach. Siegfried Koch (Fraunhofer-Institut fuer Bauphysik, Nobelstr. 12, D-70569 Stuttgart, Germany, koch@ibp.fhg.de)

There are only a few contributions to the theory of sound transmission loss (STL) of double-glazed window panes, e.g., by M. Rehfeld et al. (1996). The situation is nearly the same with the empirical approach of reducing data from laboratory measurements to a simple formula, as done by K. Goesele (1977). Goesele inferred a result in terms of the singlenumber rating according to ISO 717 based on many laboratory STL mea- surements as described in DIN 52210 and ISO 140. In particular, measurements of air-filled double-glazed window panes had been used. Meanwhile, the gas fillings changed, because the thermal insulation of the window panes had to be increased. In the last five years a large number of STL measurements of double-glazed window panes with different dimensions (pane thicknesses, gap between the panes) and gas fillings have been carried out at the Fraunhofer-Institut fuer Bauphysik. From these measurements some typical behaviors of STL versus frequency belonging to specific pane configurations could be derived. This should allow an approximate prediction of the STL for given physical dimensions of doubleglazed window panes. 
4pAA3. Sound level differences in shafts-Measurements in the IAB laboratory. Andreas Kurz, Knut Schroeter, and Wolfgang Teuber (Inst. for Acoust. and Bldg. Phys., Kiesweg 22, 61440 Oberursel, Germany)

The sound isolation between rooms can be lowered by the implementation of additional sound paths, e.g., by sound transmission via shafts. Above all, this applies to shafts which have openings to the adjacent rooms. With a normal metal duct and with certain outlets, the sound isolation was measured when a sound dampener was additionally implemented into the duct. The openings of the outlets were changed, then the level difference and the isolation index were measured. The construction of the test facility will be described.

4pAA4. Noise propagation in open-plan space with barriers. Marilda Duboc (Dept. of Mech. Eng./COPPE, Federal Univ. of Rio de Janeiro, Prédio do Centro de Tecnologia, Bloco A., Decania, Ilha do Fundão, RJ, Brasil, duboc@ct.ufrj.br) and Jules G. Slama (Federal Univ. of Rio de Janeiro, Ilha do Fundáo-RJ, Brasil)

Open-plan spaces have, in general, the distance between the floor and the ceiling much smaller to the other dimensions. This feature permits modeling sound propagation using the image source method, considering only reflection on the ceiling and the floor, which are represented by two infinite parallel planes. This paper presents some results of a computation simulation developed to represent this model. The computation program calculates the sound pressure level and the insertion loss in an open-plan space in the presence of a barrier and of a belt of absorbing material placed to the ceiling over the barrier and permits simulation of the conditions of a test method specific of open-plan spaces, the ASTMDesignation E 1111 (Standard Test Method for Measuring the Interzone Attenuation of Ceiling Systems). The results show the existence of points of weak insertion loss in the shadow zone and that a belt of absorbing material on the ceiling permits the increase of the barrier efficiency. For validation of the computation method, a comparison was made with a Kuttruff model [H. Hatanaka et al., Inter-Noise (July 1995), pp. 743-746], for the simple case without barrier and belt of absorbing material. The results have presented a good agreement.

\section{2:20}

4pAA5. An algorithm for auralization of airborne sound insulation. Rainer Thaden (Inst. of Technolog. Acoust., RWTH Aachen Templergraben, 5552056 Aachen, Germany, rth@akustik.rwth-aachen.de)

On the basis of a prediction model for airborne sound insulation (EN 12354-1), a signal processing tool was developed for auralization of indoor and outdoor noise in receiving rooms. For this purpose the direct transmission and four flanking paths were taken into account according to their one-third octave band data. These energy components were addressed to specific field situations in the receiving room with specific delays and directions. The directions of the five transmission paths as well as the reverberation tail were treated binaurally. A large number of building elements can be simulated with nine different hearing rooms. The algorithm was implemented in C (and partly in Assembler). Typical processing time for generation of a 20 -s audio file is approximately $10 \mathrm{~s}$ on a Pentium II with $233 \mathrm{MHz}$. Typical time signals, frequency responses, and some sound examples will be presented illustrating this algorithm. This work was done in cooperation with the PTB and supported by Gruenzweig and Hartmann AG.

\section{2:40}

4pAA6. Low-frequency impact sound transmission through floor systems. Alf Warnock (Natl. Res. Council, M59, Montreal Rd., Ottawa, ON K1A 0R6, Canada, alf.warnock@nrc.ca)

An extensive series of measurements of airborne and impact sound insulation on joist floors of various types was recently completed at NRC. About 190 floors with different joist types, subfloors, ceiling types, ceiling structed and sound insulation measured to frequencies lower than required by standards. The joist types comprised solid wood, wood trusses, I-joists, and steel joists. Glass, rock, and cellulose fiber sound-absorbing materials were used. Three concrete slabs were included in the series and a few of the joist floors had concrete toppings installed. Impact devices used on the floors included the standard ISO/ASTM E492 tapping machine, a walker, a JIS 1419 tire impactor, and two experimental rubber balls. The impact measurements provide an extensive, consistent set for examining alternative impact tests and possible modifications to standard tests using the ISO tapping machine. Correlations among the sound levels from the different impactors show that levels from the ISO tapping machine correlate well with those from a walker at low frequencies although the spectra are quite different in shape. The data and the prospects for a new test method and rating system will be discussed.

\section{3:00}

4pAA7. The use of agglomerated cork as an underlay for improvement of impact sound insulation in buildings. Antonio Carvalho, Constantinos Vafiadis (Acoust. Lab., Dept. of Civil Eng., College of Eng., Univ. of Porto, R. Bragas, P-4099 Porto Codex, Portugal, carvalho@fe.up.pt), and Hugo Borrego (Corticeira Amorim Industria, P-4536 Mozelos VFR, Portugal)

The cork-oak is only abundant in the Western part of the Mediterranean and is an important feature of Portuguese landscape and heritage. Portugal has about $30 \%$ of the world's cork-oak trees and accounts for more than half of the world's cork production. Agglomerated cork has natural cork granules (the by-products from the production of other cork products) as its base material. The project goal was to test and characterize the use of agglomerated cork as underlay to increase the impact sound insulation. Eight different cork agglomerate products were tested to assess their impact sound insulation quality when used as underlays with four different floor coverings (linoleum, ceramic tile, and two types of wood parquet). The measurements were made on-site (with flanking transmissions) on a coffered concrete floor without a fastened ceiling and according to International, Portuguese, and American standards (ISO-140/6 and 717, NP-669 and 2073, and ASTM-E989 and 1007). The results show that the use of this underlay improves the weighted index (Ln, w, or IIC) for each respective reference floor up to 19 (for ceramic tiles), 20 (thin parquet), 21 (linoleum), and $26 \mathrm{~dB}$ (thick parquet). Prediction formulas, determined by statistical analysis, are also presented.

\section{$3: 20$}

4pAA8. Sound irradiation from walls when excited by impact sound from fresh and waste water lines. Knut Schroeter and Wolfgang Teuber (Inst. for Acoust. and Bldg. Phys., Oberursel, Germany)

The noise produced by fresh and waste water lines is usually transmitted into neighboring walls and into rooms which should be protected. Impact sound leads to sound velocity levels which can be measured on the wall. With the help of irradiation coefficients, airborne noise levels can be calculated taking the light and heavy walls of two IAB test rooms into consideration. To vary the transmission of structure-borne sound, different pipe clamps with insulated thimbles were used. The measurement will be compared with earlier results in these test facilities. Comparisons with in situ measurements will also be made

$$
\text { 3:40-4:00 Break }
$$

\section{4:00}

4pAA9. Speech intelligibility in simulated classroom sound fields. John Bradley (IRC, Natl. Res. Council, Montreal Rd., Ottawa, ON K1A 0R6, Canada, john.bradley@nrc.ca)

Speech intelligibility in classrooms is determined by both speech/noise ratios and room acoustics factors. Using a rhyme test, speech intelligibility scores were obtained in sound fields simulated using an electroacoustic system in an anechoic room. The 16 sound fields consisted of the combi- 
nations of four levels of speech/noise ratio and four levels of a room acoustics measure giving an even distribution over all conditions likely to occur in realistic situations. The results were used to test the validity of acoustical measures that reflect the combined influence of speech/noise and room acoustics factors such as the speech transmission index, the Alcons measure, and useful/detrimental sound ratios. They were also used to investigate improved acoustical measures for speech in rooms and to evaluate the importance of speech/noise ratios relative to room acoustics factors.

\section{4:20}

4pAA10. Speech intelligibility in primary school classrooms. David J. MacKenzie (Heriot-Watt Univ., Dept. of Bldg. Eng. and Surveying, Riccarton, Edinburgh EH14 4AS, UK, D.J.MacKenzie@hw.ac.uk)

Recent research has highlighted the growing problem of noise levels in classrooms. The speech intelligibility of 60 occupied primary school classrooms has recently been investigated: high noise levels, long reverberation times, and poor classroom design are but some of the problems encountered. The recommended \%ALcons should be 5\%, whereas in some modern classrooms the value was $100 \%$. The shape of the classroom, as well as the materials used, can lead to locations within the classroom where the quality of speech heard can be poor. Measurements were carried out in classrooms before and after acoustic treatment: the speech intelligibility in treated classrooms improved noticeably. Throughout the research project children have been involved with the majority of the measurements, with their opinion being sought and listened to. The results have been used to tackle problems of poor acoustics in local schools and also in the appraisal of two new academic buildings to be constructed in Edinburgh. These buildings are for the education of children who are either hearing impaired or have a language difficulty. The design of the buildings has been carefully considered as well as the materials being used and the correct criteria for classroom acoustics.

\section{4:40}

4pAA11. Background noise levels in classrooms. Gary W. Siebein, Martin A. Gold, Mitchell Lehde, and John Ashby (Architecture Technol. Res. Ctr., P.O. Box 115702, Univ. of Florida, Gainesville, FL 32611-5702)

Field measurements of background noise levels produced by airconditioning systems were taken in a sample of 47 elementary school classrooms. Noise levels were grouped by air-conditioning system type and resulting noise criterion (NC) levels. Eight computer models of classrooms were constructed with varying combinations of absorbent materials on the surfaces of the rooms. A sound source was configured to approximate the normal speaking voice of a teacher in the room. RASTI measures were estimated for six seating locations in each room for background noise levels from $0 \mathrm{~dB}$ to NC- 55 in 5-NC point increments. RASTI values remained above 0.8 in seven of the eight rooms at NC levels of 32 and below. RASTI levels decreased to 0.50 and less in all eight rooms at levels of NC-45. The one exception to this was the room constructed with a gypsum board ceiling, vinyl tile floor, and gypsum board walls. Even with no background noise, RASTI values in this room never exceeded 0.60 . Background noise levels of NC 32 or less appear to be necessary in most typical classrooms to maintain reasonable RASTI values. Very few of the actual classrooms visited achieved these noise levels.

\section{5:00}

4pAA12. Good acoustics: A target for the new School of Architecture. Elvira B. Viveiros (Dept. of Architecture, Federal Univ. of Santa Catarina, Florianopolis, Brazil, 88040-900)

A competition for a new building for the School of Architecture is being commissioned in Florianopolis, Brazil. The project involves classrooms for theoretical classes, ateliers, library, auditorium, laboratories, staff offices, and support rooms. It consists of a 6000-sq.-m building. The site faces a noisy road, which is bound to become even noisier as the whole area develops. The provisional existing building already presents inadequacies, as for instance lecturers have complained they need to stop talking while a lorry passes along. The paper presents the proposed solutions to acclimate the new school to its environment, as well as the project details to provide good acoustic quality for the different rooms. For instance, taking into account particularities of this kind of school, absorption mechanisms play simultaneous roles in the classrooms. As the projects being developed by the students need to be hung up for oral presentation, wooden panels were planned for the side walls. Acting as vibrating membranes, the reverberation time is adequate, whilst the panels hold the posters for presentations. Solutions to avoid noise break-in are also shown.

\section{Contributed Posters}

These posters will be on display from Monday to Wednesday, 15-17 March. Authors will be at their posters from 10:00 a.m. to 12:00 noon on Wednesday, 17 March.

4pAA13. System development to reduce floor impact sound in apartment houses. Jaehee Jang, Byunghun Lim (SK R\&D Center, SK Bldg. 192-18, Chongro-Gu, Seoul, Korea, jhjang@ cosmos.skec.co.kr), and Sangwoo Lee (Kyunggi Univ., Seoul, Korea)

The floating floors used to reduce floor impact sound in European countries are not as compatible with Korean style floors because every residential floor in Korea is heated up by the floor heating method. In this case, considering the floor heat radiation effect, the cement screen is constructed to be about 40 to $50 \mathrm{~mm}$ thick. So constructing the low-density damping materials, such as glasswool, under the cement screen over 20 mm definitely makes some cracks. To prevent this kind of floor crack and to improve the impact insulation performance, the floating floor system using a plastic pallet and rubber was developed. The developed plastic pallet is $30 \mathrm{~cm}$ wide $\times 60 \mathrm{~cm}$ deep $\times 4 \mathrm{~cm}$ high and is designed to array on a concrete slab; above this plastic pallet, a 2 -cm thick thermal insulator is constructed. On this, the insulator heating coil is installed, and finally the 4-cm-thick cement screen is constructed over that. The test results of impact insulation performance for the developed system show good improvement, about $15 \mathrm{dBa}$ between before and after installation of the system.
4pAA14. Additional improvement of airborne and impact sound insulation of ceilings in prefabricated standard house-buildings. Eberhard Küstner, Peter S. Krämer, Jürgen Scholze, and Ernst Sonntag (GEBRA Gesellschaft für Bau- und Raumakustik mbH, Agricolastr. 26, D-10555 Berlin, Germany, kraemer_gebra@compuserve.com)

Earlier studies for the improvement on airborne and impact noise insulation of ceilings in prefabricated standard house-buildings has been continued by the support of the German Ministery of Building. The investigated slab blocks were erected in 1987 in Potsdam. The raw ceilings made of solid concrete have a thickness of $140 \mathrm{~mm}$. These realized ceilings have a weighted airborne sound insulation of $R_{w}^{\prime}=49 \mathrm{~dB}$ and a normalized impact-sound pressure level of $L_{n, w}^{\prime}=77 \mathrm{~dB}$ (averaged over nine measurements). A selection of floating floor materials were built in and measured: dry laser constructions (with plaster and compound cement slabs), asphalt applied by melting, and anhydrite layers. The aim of the project was to fulfill the requirements $\left(R_{w}^{\prime}=54 \mathrm{~dB}, L_{n, w}^{\prime}=53 \mathrm{~dB}\right)$ of German Standard DIN 4109 from 1989, minimizing construction height and cost. Based on earlier studies (Scholze, DAGA '94) all floating floors with a height larger than $45 \mathrm{~mm}$ (asphalt floor $40 \mathrm{~mm}$ ) have been im- 
proved to reach the requirements of DIN 4109. Using mineral wool as sound insulation material below the floating floor gave results up to $R_{w}^{\prime}$ $=56 \mathrm{~dB}$ (maximum value) and $L_{n, w}^{\prime}=48 \mathrm{~dB}$.

4pAA15. Towards a classification of acoustic comfort. A case study of a university classroom. Fausto Rodriguez-Manzo (Universidad Autonoma Metropolitana-Azcapotzalco CyAD, Av. San Pablo 180, Col. Reynosa Tamaulipas, Azcapotzalco D. F., CP 02200, Mexico, rfme@hp9000a1.uam.mx)

When it comes to speaking about acoustic comfort, one is generally dealing with a concept implying high levels of subjectivity. Hence, it becomes difficult to distinguish precisely the borderline between comfort and acoustical annoyance for an activity in a given space. Acoustical classification tools such as noise rating, noise criteria, preferred noise criteria, room criteria, balanced noise criteria, sound transmission class, and impact insulation class have proved to be partially useful in establishing averages of comfort in rooms, regardless of the spectral quality, which is intimately related to the acoustic quality of the room, and in turn related to the architectural comfort of the environment. This paper deals with the problem of correlating the quantitative aspects of acoustical measurements with elements of subjective perception. An accurate rating of the subjective acceptability of noise by polling the students, together with a simultaneous measurement of physical parameters, would result in a more realistic measure of acoustic comfort. Thus, the design of a method to correlate these parameters for the particular case of a university classroom is presented.

THURSDAY AFTERNOON, 18 MARCH 1999

ROOM MA001, 2:00 TO 6:00 P.M.

\title{
Session 4pAB
}

\section{Animal Bioacoustics: Acoustics of Echolocating Animals II}

\author{
Cynthia F. Moss, Cochair \\ Department of Psychology, University of Maryland, College Park, Maryland 20742, USA
}

Gianni Pavan, Cochair

Centro Interdisciplinare di Bioacustica e Ricerche Ambientali, Universita di Pavia, Via Taramelli 24, 27100 Pavia, Italy

\section{Invited Papers}

\section{2:00}

4pAB1. Discrimination of phantom targets by an Atlantic bottlenose dolphin (Tursiops truncatus). Roland Aubauer (Inst. of Zoology II, Univ. of Erlangen, Staudtstrasse 5, 91058 Erlangen, Germany, raubauer@biologie.uni-erlangen.de), Whitlow W. L. Au, Jeff L. Pawloski, Deborah A. Pawloski, and Paul E. Nachtigall (Hawaii Inst. of Marine Biol., Univ. of Hawaii, Kailua, HI 96734)

The ability to make fine distinctions in properties of targets is one of the outstanding characteristics of the dolphin sonar system. Behavioral experiments with these animals can reveal target echo features that can be used to model the biological sonar in order to evolve technical systems with equivalent capabilities. A phantom echo generator was developed in order to obtain programmable control over echo parameters in the time and frequency domains. Dolphin sounds are recorded with a hydrophone, transformed into the desired phantom echoes, and played back with a projector hydrophone. The signal transformation is based on the acoustic impulse response of a target and is executed on a DSP system in real time. In the first experiment the capability of a dolphin to discriminate between acoustically simulated phantom replicas of targets and their real equivalents was tested. Phantom replicas were presented in a probe technique during a materials discrimination experiment. The animal accepted the phantom echoes and classified them in the same way as it classified real targets.

4pAB2. The transmission beam pattern and echolocation signal of the harbor porpoise (Phocoena phocoena). Whitlow W. L. Au (Hawaii Inst. of Marine Biol., P.O. Box 1106, Kailua, HI 96734, au@nosc.mil), Ronald A. Kastelein, H. Tineke Rippe, and Nicole Schooneman (Harderwijk Marine Mammal Park, The Netherlands)

The transmission beam pattern of an echolocating harbor porpoise (Phocoena phocoena) was measured in both the vertical and horizontal planes. An array of seven Brüel \& Kjaer 8103 hydrophones connected to an amplifier-line driver module and a Racal Store-7 instrumentation tape recorder which was operated at 30 ips $(76 \mathrm{~cm} / \mathrm{s})$. The porpoise was trained to station in a hoop and echolocate a cylindrical target located at a range between 7 and $9 \mathrm{~m}$ while the array was located $2 \mathrm{~m}$ in front of the hoop. The beam in the vertical plane was pointed forward with a 3-dB beamwidth of approximately 19 degrees and the beamwidth in the horizontal plane was approximately $16^{\circ}$. The individual hydrophones in both the vertical and horizontal arrays measured signals that were similar in shape throughout the 40-degree span of the array. The porpoise emitted clicks with interclick intervals that were greater than the round trip travel time between the animal and the target by 20 to $35 \mathrm{~ms}$. Histogram of source level, peak frequency, and bandwidth are also presented. The average source level, peak frequency, and bandwidth were $157 \mathrm{~dB}, 128 \mathrm{kHz}$, and $16 \mathrm{kHz}$, respectively. 
4pAB3. Sperm whale clicks and the Norris/Harvey theory of click generation. Bertel Møhl (Dept. of Zoophysiology, Bldg. 131, Univ. of Aarhus, Aarhus, Denmark, bertel.moehl@biology.aau.dk)

In Odontocetes, sounds are generated within the melon, a structure homologous with the nose of terrestrial mammals. In sperm whales this nose is hypertrophied, accounting for about $1 / 3$ of the length or weight of adults. A sound-generating function has been ascribed to this organ complex [K. S. Norris and G. W. Harvey, in NASA Spec. Publ. 262, edited by S. R. Galler et al. (1972), pp. 397-417]. A sound generator weighing upward of 10 tonnes and with a cross section of $2 \mathrm{~m}$ is expected to generate high level, directional sounds. This prediction is not supported by the published data for sperm whale clicks (SL's of $180 \mathrm{~dB}$ re: $1 \mu \mathrm{Pa}$; little, if any, directionality). Such data, if truly characterizing sperm whale clicks, does not support the Norris/Harvey theory. To increase the amount of data, a hydrophone array, suspended from three platforms separated by $1 \mathrm{~km}$ and linked by radio, was deployed at the slope of the continental shelf off Andenes, Norway, in the summers of 1997 and 1998. The SL's above $210 \mathrm{~dB} \quad$ re: $1 \mu$ Pa were recorded. Also, SL differences exceeding $20 \mathrm{~dB}$ for the same click at different directions were seen. Thus, previously published properties of sperm whale clicks underestimate maximum capabilities of the sound generator and therefore cannot falsify the Norris/Harvey theory. [Work supported by the Danish National Research Foundation.]

3:00

4pAB4. Insights into the acoustic behavior of sperm whales. Gianni Pavan (Centro Interdisciplinare di Bioacustica e Ricerche Ambientali, Universita' di Pavia, Via Taramelli 24, 27100 Pavia, Italy, gpavan@telnetwork.it)

Sperm whales are still mysterious creatures. Other than to communicate, it is very likely they use sounds to navigate, to orientate, to avoid obstacles, and to find food in obscure ocean depths. Their regular clicking may serve for both communication and long-range echolocation, while codas and other patterned click sequences may serve mostly for social communication. It is known that sperm whales dive deep to search for food, probably squid and giant squid. However, to date nobody has been able to see a sperm whale detecting and capturing prey. Recordings made with towed arrays while pursuing underwater animals at close range have revealed sounds possibly used for echolocation. Accelerated click series may serve for detection and interception of prey. The patterning of clicks closely resembles the echolocation trains of dolphins, and it is very likely that sperm whales search for food in a very similar way. Bioacoustics and new technologies, such as time-depth recorders, "crittercams," and satellite-based telemetry devices may offer new and valuable insights in understanding their behavior, their survival strategies, and their relationships in the food chain. Understanding these aspects is required to define and to tune conservation strategies to offer them a chance to survive.

\section{Contributed Papers}

\section{$3: 20$}

4pAB5. Acoustic censusing of sperm whales at Kaikoura, New Zealand-An inexpensive method to count clicks and whales automatically. Olaf Jaeke (Bremer Str. 59, D-21073 Hamburg, Germany, olaf.jaeke@t-online.de) and Stephen Dawson (Univ. of Otago, Dunedin, NZ)

Special DSP software was developed to automatically count sperm whale clicks recorded along acoustic transects, and to facilitate the calibration of these counts through automated and interactive analyses of dive cycle and range experiments. Minimum pulse width and maximum interpulse interval are used as criteria for automated click detection. In addition to these criteria, interclick intervals and click amplitudes can be used for the tracing of clicks during interactive analyses. Interactive analysis of a dive of a single whale recorded under almost ideal conditions gave the following results: The mean of all interclick intervals measured within bouts of regular clicks was $0.801 \mathrm{~s}(n=2203$ intervals, cv $27.5 \%)$, the reciprocal of which corresponds to c. 1.25 clicks per second. Including 10 $\mathrm{s}$ between fluke-up and the first click recorded, silences between bouts of regular clicks, and a silence between the last regular click detected and two surface clicks found 3:37 min after the last regular click, the average click rate over a dive time of $42: 23 \mathrm{~min}$ is 0.88 per second $(n=2244$ clicks). In several dive cycles analyzed, interclick intervals were found to oscillate periodically during bouts of regular clicks. These oscillations correlated with click amplitude.

\section{3:40}

4pAB6. Specific food-related calling in dolphins. Kebkal G. Konstantin (9, Epronovskaya, 335024 Sevastopol, Ukraine, kostya@fb10.tu-berlin.de)

The results of acoustical observation of feeding behavior of bottlenose dolphins demonstrated the high degree of correlation between feeding events and very specific food signals related to every feeding act. In the paper, the features of such signals are described. They consist of specific click trains (recognizable as "creaks", or "squeaks"). Working normally for echolocation, in this case, however, the use of such click series for echolocation is physically impossible. Whether the food signals serve as an anaesthetization of a prey or merely as emotional signals is not clear. The fact is that they are in any case produced before and also shortly after the moment of catching a prey, and their intensities are high enough to provide their reliable detection even in a dolphin's back fin area. The food signals consist of some distinctive parts: (1) the stage of gaining a click's repetition rate and their power, (2) the sector with a short gap of acoustic activity, and (3) the part of highest repetition rate of low powerful clicks. Parameters of every such signal's sector may somewhat vary for different individuals staying, however, stable enough to be mathematically modeled and separated from any other click trains not related to feeding events.

\section{4:00-4:20 Break}

\section{$4: 20$}

4pAB7. The Larynx-Melon-Vestibular Lips (LMVL) model of the dolphin sonar. II. The melon beam former. James W. Fitzgerald (The Kildare Corp., 1 Spar Yard Rd., New London, CT 06320)

The LMVL model of the dolphin sonar is based on the following system components: (1) the Larynx, controlled by the palatopharyngeal sphincter and operating as a "sawtooth" relaxation oscillator, is the source of the echolocation pulses (the so-called "clicks"), and its acoustic output is coupled to the melon through the impedance matching nasal ducts; (2) the Melon, because of its low sound velocity, acts as a "slow waveguide" to form the observed forward-looking beam patterns; and (3) the residual air in the nasopharyngeal cavity acts as an air-pressure reservoir to energize relaxation oscillations of the Vestibular Lips, resulting in the lip-modulated tonal phonations (the so-called "whistles") that radiate through the corresponding vestibular sac. The larynx sawtooth generator has been treated previously [J. W. Fitzgerald, Part I Oceans '94, Vol. I, pp. 
271-276 (Sept. 1994)]. The measured sound velocity in the melon decreases with temperature from $v \cong 1.39 \times 10^{5} \mathrm{~cm} / \mathrm{s} @ 10^{\circ} \mathrm{C}$ to $v \cong 1.28$ $\times 10^{5} \mathrm{~cm} / \mathrm{s} @ 40^{\circ} \mathrm{C}$. Melonlike acoustic slow waveguides, with intermediate sound velocities, yielded average pulse beamwidths of $\theta_{1} \cong 11.5^{\circ}$ $(-3 \mathrm{~dB})$ and $\theta_{2} \cong 20.0^{\circ}(-10 \mathrm{~dB})$. This compares to accepted average values from measurements on live dolphins of $\theta_{1} \cong 10.0^{\circ}(-3 \mathrm{~dB})$ and $\theta_{2} \cong 21.8^{\circ}(-10 \mathrm{~dB})$ [W. Au, The Sonar of Dolphins (Springer-Verlag, New York, 1993), p. 105]. These preliminary measurements of melonlike acoustic slow waveguides, and the dissertation of Part I of this series, support the LMVL model of the dolphin sonar.

\section{4:40}

4pAB8. A plausible mechanism of acoustic click production in the dolphin. Nikolai A. Dubrovsky and Lyudmila R. Giro (Andreyev Acoust. Inst., Shvernika Ul. \#4, 117036 Moscow, Russia)

A mechanism of click production has been studied. Three steps were considered: experiments with Tursiops truncatus, calculations to determine the source position, and physical and mathematical models for simulation of a physical mechanism of click production. It was found that the acoustic source is situated in close proximity of the right nasal plug. A physical model of a nasal passage was composed of a rubber tube open on one end and connected to a pressurized gas container on the other. A rubber ring was put on the underwater part of the tube, which blocked the air passage at small access pressure. By varying this pressure of the elastic properties of the tube and the ring, one can put the model in a selfoscillation mode of vibrations. The displacement curve (DC) of the ring has a triangle shape and duration of about $700 \mathrm{mks}$. There were three noticeable acoustic pressure clicks with duration of 50-80 mks, phaselocked to pronounced changes in the DC. A mathematical model was developed to explain the origin of clicks and their locking to the bends of DC. The characteristics of clicks formed by the models are similar to the clicks of the dolphin.

\section{5:00}

4pAB9. Time resolution of the dolphin's auditory system: Better than 20 microseconds? Gennadi L. Zaslavski (Univ. Authority for Appl. Res. and Industrial Development, Tel-Aviv 61392, Israel)

The time resolution of the dolphin's sonar estimated as a minimum interval between echo highlights at which the dolphin is still capable of distinguishing the targets, ranges from 10 to around 300 microseconds. The problem, however, is that the target echo highlights are highly correlated and produce regularly spaced ripples in the amplitude spectrum. It is not clear whether the dolphins discriminated between a target's echo in the time or frequency domain. Unless special measures are taken to eliminate this equivocalness, the experimental results can be equally accounted for discrimination cues in either domain. Different techniques were used to eliminate frequency discrimination cues that could be used by a dolphin. The double clicks that were designed differed in interpulse interval despite their long-term amplitude spectra being identical, and the highly correlated double clicks were replaced with the uncorrelated noise double pulses [G. Zaslavskiy, Proc. I.O.A. 19, Pt. 9]. The dolphin's auditory system time resolution was found to be 25 to 30 microseconds. In this presentation the new measurement of the dolphin's time resolution of 17 to 25 microseconds will be discussed. The time resolution was estimated as a minimum delay of the click from onset of the noise or tone pulse just detectable by the dolphin.

\section{$5: 20$}

4pAB10. Improved signal processing techniques for measurement of the interpulse interval (IPI) of sperm whale clicks. Thomas J. Hayward (Naval Res. Lab., Washington, DC 20375) and G. Pavan (Univ. degli Studi di Pavia, 27100 Pavia, Italy)

Improved signal processing techniques are described for estimating the interpulse interval (IPI) associated with the multiple pulses in a sperm whale click. These pulses are believed to result from repeated reflections of the original pulse within the spermaceti organ in the whale's head. Previous estimates of the IPI have been based on time series correlation or on computation of the cepstrum of the received waveform [J. Goold, J. Acoust. Soc. Am. 100, 3431-3441 (1997)]. The present work describes real-time computation and display of the spectrogram and cepstrogram (time-cepstral distribution) [G. Pavan, Proc. IOA 19(9) (1997)] and examines the benefits of the simultaneous display of this information for field analysis and for browsing long recordings to identify sperm whale clicks. In addition, a detailed model of the spectral modulation associated with the multiple pulses is developed and leads to improved estimation of the IPI. Examples are presented that indicate the method may be more robust with respect to additive noise and the effects of surface reflection than the cepstrum-based approaches. [Work of the first author supported by ONR. Work of the second author supported by the University of Pavia, Italy, the Italian Ministry of the Environment, and by the Italian Navy.]

\section{$5: 40$}

4pAB11. Why do whales communicate at the frequencies they do? Lessons from underwater acoustic signal processing. Kevin D. Heaney (Sci. Applications Intl. Corp., 11803 Sorrento Valley Rd., San Diego, CA 92121) and Chris Clark (Cornell Univ., Ithaca, NY)

The combination of frequency-dependent attenuation of acoustic energy and the ambient noise field of the deep ocean provides a window of opportunity for acoustic communication ranging from 10 to $1000 \mathrm{~Hz}$. Why do pelagic whale species choose to use center frequencies between 20 and $50 \mathrm{~Hz}$ rather than higher frequencies in the window of opportunity? It is not a size limitation, because similar size whales which reside nearby in shallow water have center frequencies of propagation from 400 to $700 \mathrm{~Hz}$. Experience in underwater signal processing has shown that internal wave scattering can be significant for acoustic frequencies above $50 \mathrm{~Hz}$. Here the postulate is presented that deep-water whales (blues and fins) have chosen to send acoustical signals at $20 \mathrm{~Hz}$ to minimize internal wave scattering of their acoustic energy. The implications to range of detection will be made from a signal processing point of view and the impact that this would have on marine mammal biology will also be presented. Similar arguments, though based on different physics, will be employed to discuss the shallow-water whales' (bowhead, humpback, gray) choice of $400 \mathrm{~Hz}$. 


\title{
Session 4pAO
}

\section{Acoustical Oceanography: Acoustic Seafloor Classification II}

\author{
Larry Mayer, Cochair \\ Ocean Mapping Group, Department of Geodesy and Geomatics Engineering, University of New Brunswick, Fredericton, \\ New Brunswick E3B 5A3, Canada \\ Eric Pouliquen, Cochair \\ SACLANT Undersea Research Centre, Viale San Bartolomeo 400, 19138 La Spezia, Italy
}

Contributed Papers

\section{2:20}

4pAO1. Aspects of near-real time acoustic sediment characterization. Wolfgang Jans (Naval Res. Lab., Stennis Space Center, MS 39529-5004 and Federal Armed Forces Underwater Acoust. and Marine Geophys. Inst. (FWG), Klausdorfer Weg 2-24, 24148 Kiel, Germany), Warren T. Wood (Naval Res. Lab., Stennis Space Center, MS 39529-5004), and Eric Smith (Univ. of Texas, Austin, TX 78731-8029)

Quantitative near normal incidence acoustic measurements were made with a calibrated, broadband, narrow-beam echo sounder at a variety of natural sediment sites along the Florida coast. The measured reflectivity of the water-sediment interface along track lines was converted into a mapview image, using recently developed interpolation and visualization software. The computed reflectivity was then compared to sediment samples and visual observations recovered from the same area. The measurements demonstrated that areas of different bottom type within a site could be clearly distinguished using acoustic techniques. At some sites, conventional expectations that softer sediments show a lower reflectivity than harder sediments were fulfilled. However, at other sites, the expectation superficially appears to be violated presumably due to the influence of bottom roughness. The presence of turtle grass also reduces reflectivity. To gain an understanding of these findings, histograms of the received echo signal and frequency spectra for different areas have been compared. The rapid 2.5-D visualization of bottom reflectivity and bathymetry has been shown to provide improved capability for near-real time decisions in seafloor investigations. [Work supported by ONR and BWB.]

\section{2:40}

4pAO2. Comparison of seafloor character as measured by calibrated vertical-incidence profiler and sidescan sonar. Peter Fleischer (Code 7431, Naval Res. Lab., Stennis Space Center, MS 39529), Wolfgang Jans (Naval Res. Lab. and Federal Armed Forces Underwater Acoust. and Marine Geophys. Inst. (FWG), 24148 Kiel, Germany), and Warren T. Wood (Naval Res. Lab., Stennis Space Center, MS 39529)

Sidescan sonar backscatter from a variety of continental shelf bottom types reveals considerable divergence from vertical-incidence reflection coefficients. Acoustic data are from a $300-\mathrm{kHz}$ sidescan sonar and a calibrated, constant beam width, $10-50 \mathrm{kHz}$ vertical incidence profiler, and are supported by extensive physical, geoacoustic, textural, and morphologic measurements. Bottom types include a wide range of quartzose and carbonate sands and silts, seagrass, coral heads, coral reef, and hardbottoms. The two systems show notable divergences over seagrass and carbonate hardbottoms, which produced low normal-incidence reflection co- efficients but high backscatter from sidescan sonar, and smooth, packed sands, which gave high normal-incidence reflection coefficients but low sidescan backscatter. The divergences appear to be caused by bottom roughness. Narrow-beam, vertical incidence systems are subject to signal loss from out-of-beam scattering over rough bottoms, whereas roughness increases backscatter at the low grazing angles over which the sidescan system operates. Although both systems will accurately map bottom character, there is no direct correspondence betwen the two vis-à-vis bottom type. Therefore, to extend seafloor classification from vertical incidence profiling into an areal approach with sidescan sonar requires more than simple lateral extrapolation. [Work supported by ONR.]

\section{3:00}

4pAO3. Effects of differing sediment types and structures on normal incident acoustic reflections. Warren T. Wood (Naval Res. Lab., Stennis Space Center, MS 39529), Wolfgang Jans (Naval Res. Lab. and Federal Armed Forces Underwater Acoust. and Marine Geophys. Res. Inst. (FWG), 24148 Kiel, Germany), and Eric Smith (Univ. of Texas, Austin, TX 78731-8029)

Data acquired during a recent survey of coastal Florida and the Bahamas have provided an opportunity to correlate normal and near normal incidence acoustic returns with distinguishing seafloor sediment characteristics. These include physical property measurements (both in situ and laboratory) as well as bottom textural measurements derived from diver observations and from video and stereo photography. Measurements were made with a calibrated 1-3 piezocomposite transducer, which offered a very narrow ( $~ 8 \mathrm{deg}$ ) beam width over the $10-50-\mathrm{kHz}$ transmitted chirp. In addition, a roll and pitch sensor mounted on the transducer and 0-5 deg ship motion allowed for a monostatic backscatter experiment. This resulted in quatitative measurements of reflectivity versus angle over a relatively broad frequency band. Interestingly, the measured reflection coefficients were significantly lower (especially at high frequencies) and exhibited a greater range in values than those obtained in laboratory tank measurements with a flat, level interface. Also, the range of reflectivity at any fixed angle and anchor location was greater than the systematic dependence on angle. This suggests that very slight ship motion combined with either bottom roughness or discrete scatterers is responsible for the variable reflectivity. [Work supported by ONR.] 
process is nonlinear, but in practice it is well behaved and converges quite

4pA04. In situ, laboratory, and modeled permeability of unconsolidated sands. Dawn L. Lavoie (Code 7431, Naval Res. Lab., Stennis Space Center, MS 39529), Allen Reed (Univ. of Southern Mississippi, Stennis Space Center, MS 39529), and Keven Briggs (Naval Res. Lab., Stennis Space Center, MS 39529)

Bulk physical sediment properties, especially permeability, are required to predict sediment geoacoustic and geotechnical behavior. Of the variables controlling permeability in surficial sediments, pore space distribution is probably the most significant. The objective is to quantify the relationship between sediment microfabric and sediment flow properties. Relatively undisturbed samples were collected by divers from the upper 25 $\mathrm{cm}$ of seafloor from several locations on the Florida shelf and near Bimini in the Bahamas. The undisturbed sediment microfabric was maintained by impregnating the samples using a polyester resin. Sediment microfabric was examined using petrographic and scanning electron microscopes. Representative images were scanned and analyzed using Image Tool and Image Tool Plug Ins. Using erosion-dilation analysis, the $2 \mathrm{D}$ imaged pores and pore network were defined and quantified. CT images were used to determine sediment isotropy and to generate 3D structural images, which were used as input to effective medium theory to predict single-phase flow within the sediment. The modeling results are compared to laboratorymeasured permeability from the same samples, and with permeability measured in situ using a falling head permeameter. [This work was supported by the Naval Research Laboratory.]

\section{3:40}

4pA05. Subsurface classification of shallow gassy river sediments using broadband seismic data. Stephen F. Bloomer, N. Ross Chapman (School of Earth and Ocean Sci., Univ. of Victoria, P.O. Box 3055, Victoria, BC V8W 3P6, Canada), William T. Collins (Quester Tangent Corp., Sydney, BC V8L 5Y8, Canada), and Peter G. Simpkin (INRS-Océanologie, Rimouski, QC G5L 3A1, Canada)

Acoustic seabed classification from sonar data has generally focused on attributes of the return echo from the seafloor. For many shallow-water engineering applications, such as dredging, detailed knowledge of the shallow sub-bottom is also required. Consequently, acoustic classification of the sub-bottom is also often essential. In 1995, a shallow-water, highresolution seismic predredging survey was conducted on the St. Lawrence River in water depths of 10-15 m using the IKB SEISTEC high-resolution profiler, which has a bandwidth of $1-12 \mathrm{kHz}$ and subsurface penetration in excess of $10 \mathrm{~m}$. In these river sediments, it was found that shallow gas had a controlling effect on the appearance of the seismic sections in some places. The shallow gas could often be identified as a curtain in which there are no acoustic returns below a strong subsurface reflector with a phase reversal relative to the seafloor reflection. The frequency dependency of the ratio of the signal amplitude between the seafloor and subbottom reflectors may also be diagnostic of gas reflectors in that, instead of attenuation, resonances may occur. Using the spectral-ratio method, the attenuation between successive reflectors characteristic of the sediment between the reflectors can be determined. Results of classifying subsurface sediments between distinct reflectors from this survey will be presented.

\section{4:00}

4pA06. Acoustic inversion for Biot parameters in water-saturated sand. Nicholas P. Chotiros (Appl. Res. Labs., Univ. of Texas, P.O. Box 8029, Austin, TX 78713-8029)

Using an inversion of Biot's theory, based on the formulation by Stoll, operative values of grain bulk modulus and the frame bulk and shear moduli of water-saturated sand may be extracted from measurements of compressional and shear wave speeds and reflection loss. The inversion rapidly to unique values. The term grain bulk modulus, as defined in the formulation by Stoll, is not necessarily identical to the bulk modulus of the grain material, as is often assumed. Examples of the inversion will be given, and the significance of the operative values of the grain bulk modulus will be discussed. [Work supported by Office of Naval Research: Ocean Acoustics.]

\section{4:20-4:40 Break}

\section{$4: 40$}

4pA07. Acoustic classification of a shell-covered seafloor. Timothy K. Stanton (Dept. of Appl. Ocean Phys. and Eng., Woods Hole Oceanogr. Inst., Woods Hole, MA 02543-1053)

Acoustic scattering by the seafloor is sometimes influenced, if not dominated, by the presence of discrete volumetric objects such as shells. Classification of a shell-covered seafloor requires understanding of the acoustic scattering properties of the shells. A series of measurements of target strength of a type of benthic shelled animal and associated scattering modeling have recently been completed. In addition, a simple formula has been derived that expresses the area scattering strength of the seafloor in terms of the reduced target strength (RTS) of the discrete scatterers and their packing factor (where RTS is the target strength normalized by the size of the scatterers). The formula shows that, to first order, the backscattering at high acoustic frequencies by a dense layer of shells (or other discrete bodies such as rocks) depends principally upon material properties of the objects and packing factor, and is independent of size and acoustic frequency. There are reasonable comparisons between scattering data from shell-covered sections of seafloor and predictions using this formula and the measurements/modeling of shell target strength. The functional dependence of this and other seafloor scattering formulas upon shell and shell-bed characteristics are discussed to assess one's ability to classify a shell-covered seafloor. [Work supported by ONR.]

\section{5:00}

4pA08. Scaling regimes in incoherent surface-reflected and in-sediment sound fields. Eric Smith (Appl. Res. Labs., Univ. of Texas, Austin, TX 78713-8029), Michael D. Richardson, Warren T. Wood (Naval Res. Lab., Stennis Space Center, MS 39529-5004), and Wolfgang Jans (Naval Res. Lab. and Federal Armed Forces Underwater Acoust. and Marine Geophys. Inst. (FWG), Klausdorfer Weg 2-24, 24148 Kiel, Germany)

A suite of collocated measurements of broadband in-sediment acoustic propagation and surface reflection was made at nine sites on the Florida shelf and in the Gulf of Mexico, between 16 August and 6 September 1998. Closely spaced in-sediment transmission measurements, and populations of narrow-beam echo soundings, provided ensembles from which mean and incoherent components of propagating and reflected sound fields were derived. The two components contain mutually independent information about the effective bulk properties and heterogeneity of the medium. Attenuation of the direct arrival through the sediment depends on both intrinsic absorption and scattering from heterogeneities. Temporal decay of the incoherent "coda" at a point, not described by homogeneous effective-medium theory, requires scattering to maintain sound energy in a region. Incoherent coda in the surface reflection can be generated either from multiple scattering near the surface, or random reflection within the sediment bulk. Scaling regimes of both kinds of attenuation will be demonstrated and compared, between in-sediment propagation and surface reflection, and among different sites. As a control, scaling of the coda decay constant will also be compared to results of reflection from sand in an indoor tank, from which stratification and surface roughness were removed. [Work supported by ONR.] 
to extract descriptive parameters of a seabed type. Both direct methods,

4pA09. Seabottom roughness study using a hydrosweep-multibeam system. Bishwajit Chakraborty, Vijay N. Kodagali (Natl. Inst. of Oceanogr., Dona Paula, Goa: 403 004, India, bishwajt@csnio.ren.nic.in), and Hans W. Schenke (Alfred Wegener Inst. for Polar and Marine Res., 27515 Bremerhaven, Germany)

Seabottom profiling using a multibeam echosounder is a well-known method to acquire a high-resolution and high-density data set for bathymetric mapping of survey area. The use of multibeam echosounder backscatter signals for bottom roughness characterization is a modern technique. Here, the model results of seabottom backscatter data using a hydrosweep-multibeam system, from some of the geologically wellknown areas of Southern Oceans, are presented. Using the capabilities of multibeam systems, angular backscatter strengths are determined employing various corrections. Different bottom backscattering modeling techniques like the composite roughness [Jackson et al., J. Acoust. Soc. Am. 79, 1410-1422 (1986)] and two-layer Helmholtz-Kirchhoff model [Talukdar et al., J. Acoust. Soc. Am. 97, 1545-1558 (1995)] for estimation of bottom roughness is applied. Various seabottom parameters like rootmean-square (rms) relief height, correlation lengths, attenuation coefficients, and layer thickness using the two-layer Helmholtz-Kirchhoff model are calculated. The interface roughness parameters, i.e., slope and intercept values, and volume roughness parameters are computed using the composite roughness theory for the same geological areas.

\section{$5: 40$}

4pAO10. The comparison of inverse filtering techniques applied together with fractal analysis in the sea bottom recognition. Zbigniew Lubniewski, Marek Moszynski, and Andrzej Stepnowski (Tech. Univ. of Gdansk, Acoust. Dept., ul. Narutowicza 11/12, 80-952 Gdansk, Poland, lubniew@eti.pg.gda.pl)

The results of the combined approach using fractal analysis and inverse filtering methods applied to sea-bottom-type recognition are presented. The single-beam one-frequency echosounder was used in described investigations. The bottom-scattering impulse response was used including windowed SVD, regularization and wavelet analysis, as well as iterative techniques like maximum entropy, and expectation, maximization, and smoothing (EMS) were investigated. The data were acquired from acoustics surveys on Lake Washington and Liberty Bay. The results were compared and discussed especially in the context of excessive smoothing of the extracted fractal features by applied newly developed inverse techniques.

\section{6:00}

4pAO11. Seafloor scattering: Modeling and analysis of classification clues. Anatoliy N. Ivakin (Andreev Acoust. Inst., Shvernika 4, Moscow 117036, Russia, aniva@glasnet.ru)

Possibilities of scattering data inversion for various seabed properties are analyzed using recently developed models of acoustic bottom interaction. Different scattering mechanisms are considered giving the main contributions to total bottom scattering which are due to different types of medium irregularities: volume inhomogeneities of the sediment medium and roughness of its surface and internal interfaces. High-frequency model of scattering corresponds to sufficiently strong absorption and small penetration into the sediment such that the influence of seabed stratification is negligible. At lower frequencies, a layered seabed scattering model considers also refraction and interference effects due to sediment stratification as well as effects of elastic scattering and reflection from the basement supporting both compressional and shear waves. Frequency-angular dependencies of the scattering strength for both monostatic and bistatic cases are calculated and analyzed for various seabed types. Distinctive features are discussed which can be used as classification clues for determination of various parameters of the seafloor. Additional clues are revealed from an analysis of the spatial correlation function of the scattered field that can be used for distinguishing and/or separating the volume and roughness components of scattering.

\section{Contributed Posters}

These posters will be on display from Thursday to Friday, 18-19 March. Authors will be at their posters from 10:00 a.m. to 12:00 noon on Friday, 19 March.

4pA012. Generalized additive models for classifying sea-bottom types. Patrick Schneider (Instituto de Ciencias del Mar, Barcelona, Spain) and John Hedgepeth (BioSonics, Inc., Seattle, WA)

One encounters many types of sea-bottom, a continuous spectrum of all kinds of transitions between one category and another, not just clearcut categories. A number of parameters, which the BioSonics Visual Bottom Typer extracts from the data, are looked at as a continuum, from one bottom type to another. As generalized additive models (GAMs) are nonlinear regression models, they can be useful to find relating functions for some of the parameters, significant for different bottom characteristics. The advantage of the GAMs is that if there should be a linear relation between two parameters, they are also able to reveal this, because a linear model is included as one special case in a GAM. Also, there can be problems with GAMs: There may not be a single transition from, e.g., soft/muddy to hard/rocky, but different kinds of bottom types can be connected in arbitrary ways; building a library from known bottoms, as has previously been done, might be the easiest and most practicable way.
4pAO13. Bottom layers' structure influence on ocean shelf water low-frequency source sound field numerical modeling. Andrew G. Semenov, Alexander M. Derzjavin, and Oleg V. Kudryavtzev (N. N. Andreev Acoust. Inst., Russian Acad. of Sci., 4 Shvernik St., 117036 Moscow, Russia)

The low-frequency vector wave field modeling method for the prediction of fields in layered inhomogeneous ocean and the ocean bottom environment is proposed. The method is especially stable to vertically cut media multiple arbitrary thickness layers. Based on integral presentation of 2-D cylindrically symmetric source wave fields in elastic media, the method accounts for all wave types involved. Inhomogeneous media in the problem are cut in $N$ horizontally homogeneous layers, for which $4(N$ $-1)$ equations were derived, accounting for conditions on both layers boundaries. Equation factors found by the Schmidt global matrix method are introduced in Fourier-Bessel integral expressions for local field parameters. Expressions were calculated numerically to obtain values of 
acoustic pressure and elastic stress, vertical and horizontal particle velocity component in liquid and elastic media, respectively. Deviations fromexact solutions expressed in modeling stability losses were obliged mainly to layers' excessive thickness choice. A simple enough "benchmark' problem solution, where results for the conventional and the pro- posed method were compared to the exact solution, demonstrates method advan-tages. Point source of frequency $0.01-10.0 \mathrm{~Hz}$, situated in a waterlayer field model is presented. The influence of the bottom elastic layers' structure on model parameters is demonstrated. The geological-acoustic bottom model depending on frequency is proposed.

THURSDAY AFTERNOON, 18 MARCH 1999

ROOM MA043, 2:00 TO 6:20 P.M.

\title{
Session $4 p B B$
}

\section{Biomedical Ultrasound/Bioresponse to Vibration: Lithotripsy}

\author{
Robin L. Cleveland, Cochair \\ Department of Aerospace and Mechanical Engineering, Boston University, Boston, Massachusetts 02215, USA \\ Michael Delius, Cochair \\ Institute for Surgical Research, University of Munich, Klinikum Grosshadern, D-81366 Munich, Germany
}

\section{Invited Papers}

\author{
2:00
}

4pBB1. Effect of increased ambient pressure on lithotripsy-induced cavitation in bulk fluid and at solid surfaces. Michael R. Bailey (Appl. Phys. Lab., College of Ocean and Fishery Sci., Univ. of Washington, 1013 NE 40th St., Seattle, WA 98105), Robin O. Cleveland (Boston Univ., Boston, MA 02215), Oleg A. Sapozhnikov (M. V. Lomonosov Moscow State Univ., Moscow 119899, Russia), James A. McAteer, James C. Williams, Jr. (Indiana School of Medicine, Indianapolis, IN 46202-5120), and Lawrence A. Crum (Univ. of Washington, Seattle, WA 98105)

By reducing cell damage but maintaining stone comminution, overpressure (OP)—increased hydrostatic pressure—offers the promise of safer more effective shock wave lithotripsy (SWL) [Delius, UMB 23, 611-617]. A current hypothesis is that stones offer cavitation sites - sites of violent bubble activity - when the cavitation nuclei in a free field have been dissolved into the fluid by overpressure. Cavitation around solid bodies and in a free field with and without overpressure is investigated. High-speed photography and dual passive cavitation detection (PCD) were used to measure the temporal and spatial extent of cavitation in a 10 - $\mathrm{cm}^{3}$ plastic chamber that minimally altered the shock wave. In a free field at $\mathrm{OP}=0 \mathrm{~atm}$, cavitation bubbles formed and collapsed in $280 \pm 20 \mu \mathrm{s}$. The time of collapse $t^{c}$ was halved at $\mathrm{OP}=1 \mathrm{~atm}$, and cavitation activity was not detectable at $\mathrm{OP}=3 \mathrm{~atm}$. With a stone present, a bubble $\sim 10$ times larger than free-field bubbles grew on the surface of the stone and collapsed after $440 \pm 50 \mu \mathrm{s}$. With overpressure $t_{c}$ shortened cavitation was still measurable $\left(t_{c}=80 \pm 12 \mu \mathrm{s}\right.$ at $\left.\mathrm{OP}=3 \mathrm{~atm}\right)$. Results were similar with human kidney stones, artificial cement stones, and an aluminum plate, and support the caviation hypothesis. [Work supported by NIH DK43881, FIRCA, and CRDF.]

4pBB2. Clinical evaluation of cavitation thresholds in patients undergoing lithotripsy. Andrew J. Coleman, Prashant K. Verma (Dept. of Med. Phys., St. Thomas' Hospital, Lambeth Palace Rd., London, UK), and Mark D. Cahill (Univ. of Bath, Claverton Down, Bath, UK)

Thirty patients undergoing extracorporeal lithotripsy for renal and uteretic stone disease were included in a study of cavitation thresholds. A passive, focused hydrophone was used to detect the 1-MHz component of the broadband noise emmission from bubbles generated at depth in tissue close to the focus of a Stortz Monolith SL20 lithotripter. A theoretical model was used to obtain predictions of the peak negative pressure at the focus of the lithotripter at output settings corresponding to those used clinically. A detectable cavitation threshold was identified in 18 patients. As the output level of the lithotripter is reduced, multiple bubble collapses cease to be detected below a well-defined threshold which is similar in all patients. There is also some evidence that this threshold is dependent on the previous exposure history of the tissue. [Work supported by MRC.]

4pBB3. Relationship of shock wave lithotripsy (SWL)-induced lesion size to renal hemodynamics at differing SWL parameters. Andrew P. Evan (Dept. of Anatomy, Indiana Univ., School of Medicine, MS 208, 635 Barnhill Dr., Indianapolis, IN 46202), Lynn R. Willis, Bret A. Connors, Philip Blomgren, and James E. Lingeman (Methodist Hospital, Inc., Indianapolis, IN)

The present study was designed to determine the relationship between lesion size and hemodynamic changes by altering the $\mathrm{kV}$ level, shock number, and pre-treatment status of the kidney. Six-week old pigs were divided into groups based on treatment protocol: 12,18 , and $24 \mathrm{kV}$ at 2000 shocks, $24 \mathrm{kV}$ at 8000 shocks, pyelonephritis $24 \mathrm{kV}$ at 2000 shocks and sham. Following anesthesia each pig was prepared for clearance determinations of glomerular filtration rate, renal plasma flow, and PAH extraction. The lower pole of one kidney was treated with an unmodified HM3 lithotripter except the sham group. Renal function was measured $1 \mathrm{~h}$ before and 1 and $4 \mathrm{~h}$ after SWL followed by renoval of both kidneys for morphological analysis. Lesion size increased as $\mathrm{kV}(0.27 \pm 0.1 \%$, $2.28 \pm 0.3 \%, 6.1 \pm 1.7 \%$, respectively) and shock number $(6.1 \pm 1.7 \%, 13.6 \pm 1.4 \%)$ increased. Renal hemodynamic changes oc- 
curred independent of lesion size. Pyelonephritis potentiated the hemodynamic changes seen with 2000 shocks and therefore, more closely mimicked 8000 shocks. Thus two separate mechanisms may be responsible for vascular and tubular injury induced by SWL, and that pre-existing renal disease may potentiate the lesion induced by a clinical dose of SWL. [Work supported by NIH Grant No. PO1 DK43881.]

3:00

4pBB4. Controlled and forced collapse of inertial cavitation bubbles during SWL. Pei Zhong and Xufeng Xi (Dept. of Mech. Eng. and Mater. Sci., Duke Univ., Box 90300, Durham, NC 27708)

The dynamics of inertial cavitation induced by current clinical lithotripters is uncontrolled. Recently, it has been shown [Zhong et al., J. Urol. 158, 2323-2328 (1997)] that a second shock wave generated in a few hundred microseconds following the standard lithotripter pulse can be used to control and force the collapse of cavitation bubbles toward the target stones, leading to increased stone comminution. Different approaches of generating the second shock wave have been tested, including combination of two EH generators and modification of ellipsoidal reflectors. To provide a unit that can be combined with clinical EH lithotripters, a piezoelectric annular array (PEAA) generator was designed and fabricated. This PEAA generator consists of eight 50-mm PZT4 elements of $200-\mathrm{kHz}$ resonant frequency, which produce a peak compressive pressure of $\sim 8 \mathrm{MPa}$ with a $-6-\mathrm{dB}$ beam diameter of $5 \mathrm{~mm}$. A fiber-optic link was used to trigger the release of the PEAA generator (jitter less than $10 \mu \mathrm{s}$ ). The combined shock-wave generator, with optimal time delay between the release of the lithotripter and the second pulses, was found to increase stone fragmentation in vitro by $48 \%$ to $67 \%$, as compared to the standard lithotripter. [Work supported by NIH RO1 DK 52985.]

$3: 20$

4pBB5. Shock wave-inertial microbubble interaction. Pei Zhong, Xufeng Xi, Songlin Zhu (Dept. of Mech. Eng. and Mater. Sci., Duke Univ., Box 90300, Durham, NC 27708, pz1@me1.egr.duke.edu), and Haifan Lin (Duke Univ., Durham, NC 27710)

To produce in situ shock wave-inertial microbubble interaction, an annular ellipsoidal reflector, confocal but $15 \mathrm{~mm}$ shorter in major axis than the ellipsoidal reflector of a XL-1 lithotripter, was fabricated and combined with the XL-1 reflector. With this modification, a small portion of the spark-generated spherical shock wave is reflected and diffracted by the annular reflector, producing a weak shock wave approximately $8.5 \mu \mathrm{s}$ in front of the lithotripter pulse. This preceding shock wave $(-0.96<P<$ $-1.91 \mathrm{MPa}$ at $25 \mathrm{kV}$ ) induces inertial microbubbles, which expand to a maximum size of 100 to $200 \mu \mathrm{m}$ before being collapsed in situ by the ensuing lithotripter pulse. High-speed shadowgraph imaging and passive cavitation detection have revealed strong secondary shock wave emission immediately following the propagating lithotripter shock front and microjet formation along the wave propagation direction, features that are absent from the standard lithotripter pulses. With optimal pulse combination, membrane permeabilization of mouse lymphoid cells produced by the modified shock waves is substantially enhanced (up to $91 \%$ ) at low dosage $(<50$ shocks), and cell injury is significantly increased (up to 50\%) at high dosage ( $>100$ shocks), compared to standard lithotripter pulses. [Work supported by NIH R21 HL60327.]

\section{$3: 40$}

4pBB6. Transfer of an exact number of molecules into cells by shock waves. M. Delius and G. Adams (Inst. for Surgical Res., Univ. of Munich, Klinikum Grosshadern, 81366 Munich, Germany, delius@icf.med.uni-muenchen.de)

Shock wave application to cells in vitro causes a transient increase of the permeability of the cell membrane which does not lead to cell death. The transfer of molecules into the cytosol was quantified with florescein dextrans by flow cytometry. It was much higher than the uptake by endocytosis. It did not change over the next hours; there was no efflux. It depended linearly on the dextran concentration in the medium. In addition, transfer of a low number of dextran molecules of high molecular weight was equal to a high number of low weight. In conclusion, shock wave transfer reflects directly the amount of fluid transferred. It is in the femtoliter range. The number of molecules acoustically transferred by a certain fluid volume into a cell depends on their concentration in the medium. The protein gelonin was used to transfer different numbers of molecules into the cytosol and to assess the cell proliferation. Acoustic transfer of a single molecule or of any other defined number of molecules into the cell is a simple method to assess dose-response effects. It might be widely applied in disciplines such as physiology, biochemistry, and drug design.

4:00

4pBB7. Aspects of pressure pulse lithotripter measurements. Friedrich Ueberle (Dornier Medtech, Industriestrasse 15, 82110 Germering, Germany)

Although pressure pulse waves have been used as the standard treatment of kidney stones (ESWL) for the last 15 years, the relevance of their different physical parameters is not fully understood. The new approach of tissue treatment for pain reduction (ESWA, ESWT) raises even more questions. In 1998, the IEC document 61846 "Ultrasonic-pressure pulse lithotriptersCharacteristics of fields" was internationally accepted. It contains the descriptions of all the parameters which may be measured, as well as the different hydrophone types which may be used. Additional work was done, e.g., by the FDA and in the "German society for shockwave lithotripsy" (DGS) to define a standard set of pressure pulse data, measured according to the IEC standard. The standard parameters may be measured using different hydrophone types and using either (only) the positive parts of the waves or the complete wave, including rarefaction parts for the calculation of energy parameters. Choosing a hydrophone capable of serving the demands of lifetime, signal confidence (including time parameters and rarefaction pressure), as well as cost efficacy is the most delicate task. Various aspects necessary for the interpretation of standard parameters measured using different hydrophones will be discussed. 


\section{Contributed Papers}

\section{4:40}

4pBB8. In vivo modeling of the HM3 lithotripter. Robin O. Cleveland (Dept. of Aerosp. and Mech. Eng., Boston Univ., Boston, MA 02215)

A computer model for the propagation of shock waves in the HM3 lithotripter has recently been developed using the KZK equation [Averkiou and Cleveland, J. Acoust. Soc. Am., submitted]. The model has been extended to account for propagation through inhomogeneous tissue. The tissue was modeled as a layered medium with properties varying in the direction of acoustic propagation. Tissue absorption was modeled as consisting of four relaxation processes which provided an excellent fit to empirical absorption characteristics. Results were compared to waveforms measured in pigs [Cleveland et al., Ultrasound Med. Biol. 24, 293-306 (1998)]. Measured and calculated waveforms were in good agreement, including the rise time of the shock. However, at the focus measured, peak pressure was less than calculated numerically. It is supposed that smallscale arbitrary inhomogeneities, that the model does not account for, produced aberration effects that lead to the reduction in measured amplitude. The model predicted large negative pressures in the perirenal fat where cavitation events have been detected. [Work supported by NIH PO1DK43881.]

\section{5:00}

4pBB9. Renal vasoconstriction caused by SWL to one pole of one kidney may attenuate the injury caused by subsequent SWL to the other pole of that kidney. Lynn R. Willis (Dept. of Pharmacology and Toxicology, Indiana Univ., School of Medicine, MS A528, 635 Barnhill Dr., Indianapolis, IN 46202), Andrew P. Evan, Bret A. Connors, Philip Blomgren, and James E. Lingeman (Methodist-Clarian Hospital, Inc., Indianapolis, IN)

These experiments examined the size of the renal lesions produced by SWL after 2000 shocks applied first to one pole and then immediately to the other pole of the same kidney. Anesthetized, 6-week-old pigs received 2000 shock waves at $24 \mathrm{kV}$ (Dornier HM3) first to the lower pole and then to the upper pole of the same kidney (4000 shocks in all). Bilateral measurements of renal blood flow (RBF) were obtained $1 \mathrm{~h}$ before and 1 and $4 \mathrm{~h}$ after SWL. Lesion sizes in each pole were determined by serial sectioning and digital photography. SWL successively to lower and then upper poles in each of two pigs caused marked bilateral renal vasoconstriction (reduction of RBF). In one pig, the upper-pole lesion was only about $5 \%$ the size of the initial lower-pole lesion. In the second pig, the lesions in the upper and lower poles were of similar sizes. In both pigs, the combined lesion sizes after 4000 shocks $(6.81 \%$ and $5.72 \%$, respectively) were nearly the same as the size of lesions produced by a single application of 2000 shocks to only one pole $(6.1 \pm 1.7)$. The data suggest that renal vasoconstriction limits the renal injury caused by SWL.

\section{$5: 20$}

4pBB10. Shot-to-shot variability of acoustic axis of a spark-source lithotripter. Oleg A. Sapozhnikov (Dept. of Acoust., Phys. Faculty, Moscow State Univ., Moscow 119899, Russia, olegs@na.phys.msu.su), Michael R. Bailey, and Lawrence A. Crum (Univ. of Washington, Seattle, WA 98105)

The shot-to-shot spatial variability of acoustic and cavitation fields of a spark-source lithotripter was investigated. Spark jitter, variation in the arc strength and location is cited for lack of repeatability in laboratory experiments and is thought to contribute to the success of the Dornier HM3 in comminuting kidney stones, which tend to move during treatment. Transverse variability of the acoustic focus was assessed by measuring the location of the shock wave (SW) induced fountain. The water bath was lowered and a small cup half-filled with ink and having a gridded paper roof was placed at the level of the focus. An ink drop created by the fountain was recorded on the paper. Drop diameter varied from 0-12 mm, location of its center varied by about $1 \mathrm{~mm}$. The extent and location of the cavitation field was measured by high-speed camera. The cavitation cluster was $1 \times 10 \mathrm{~cm}$ at its largest extent but bubbles collapsed in a narrow 1-2 $\mathrm{mm}$ line. The location of the line was repeatable to within $2 \mathrm{~mm}$. The results indicated that spark jitter affected SW amplitude but had little effect on the location of the acoustic axis. [Work supported by NIH (DK 43881), CRDF and RFBR.]

$$
5: 40
$$

4pBB11. Use of two pulses to localize and intensify cavitation in lithotripsy. Dahlia L. Sokolov, Michael R. Bailey, and Lawrence A. Crum (Appl. Phys. Lab., College of Ocean and Fishery Sci., Univ. of Washington, 1013 NE 40th St., Seattle, WA 98105)

A single electrohydraulic lithotripter pulse excites a cigar-shaped cavitation field that plays a role in both stone comminution and tissue damage during kidney stone treatment. The diameter and length of such a field (in water) are approximately 1 and $10 \mathrm{~cm}$, respectively, and the lifetime is $\sim 500 \mu \mathrm{s}$. A second, time-delayed pulse can be used to suppress cavitation during growth. As a result, by using two lithotripters facing each other and firing simultaneously, a cavitation field can be created for which the lithotripsy pulses sum coherently at the center to enhance cavitation and counteract each other off-center to suppress cavitation. The result is an intense and localized cavitation field, contained entirely by a cylinder of diameter $\sim 3$ and length $\sim 4 \mathrm{~cm}$, and having a lifetime twice that of a single-pulse field. When produced in vivo, such a localized cavitation field may increase the rate of stone comminution while mitigating damage to surrounding tissue. Experiments were undertaken in vitro with a Dornier HM3 experimental lithotripter, using a Kodak EktaPro 4540 digital highspeed motion analyzer for data acquisition. [Work supported by NIH PO1 DK43881.]

\section{6:00}

4pBB12. Generation of a very high pressure pulse at the surface of a sandwiched piezoelectric material. Jean-Pierre Sferruzza and Dominique Cathignol (INSERM Unite 281, 151 Cours Albert Thomas, 69424 Lyon Cedex 03, France, cathignol@lyon151.inserm.fr)

New clinical concepts in lithotripsy demand small size shock heads. Reduction of piezoelectric shock head is only possible if accompanied by a corresponding increase of the pressure generated at the surface of each transducer, so that the total pressure at the focus remains the same. Because the pressure generated by a transducer is proportional to the electric field applied between its two electrodes, a higher pressure could be generated by using greater electric fields. Nowadays, the value of the usual fields is no more than $2 \mathrm{kV} / \mathrm{mm}$ and the generated pressure at the surface is about 10 bars. Unfortunately, higher pulsed fields applied on the transducer lead to breaking phenomena. For the first time, a proposal is presented to increase the pressure using sandwiched transducers, which are a combination of several stacked transducers excited at such times that the pressure waves generated by each one are fully added at the interface transducer-propagating medium. This new and patented technique has been successfully tested. More than 30 bars were obtained with a twolayer transducer each 5-mm thick, and working under an $8-\mathrm{kV}$ excitation voltage. Using this new elementary transducer a piezoelectric shock head of only $20-\mathrm{cm}$ diameter is expected. 


\title{
Session 4pEAa
}

\section{Engineering Acoustics and Signal Processing in Acoustics: Digital Signal Processing for Hearing Aids II}

\author{
Roger T. Richards, Cochair \\ Naval Undersea Warfare Center, 1176 Howell Street, Newport, Rhode Island 02841, USA \\ Juergen Peissig, Cochair \\ Sennheiser electronic, 30900 Wennebostel, Germany
}

\section{Contributed Papers}

\section{2:00}

4pEAa1. Acoustical analysis on the performance of digital hearing aids. Yingyong Qi and Andrew Dittberner (Univ. of Arizona, P.O. Box 210071, Tucson, AZ 85721, yqi@u.arizona.edu)

The acoustic spectral distances were computed for phonemes, /p/, /t/, and $/ \mathrm{k} /$ recorded under a variety of recording conditions, which include: a low (50 dB SPL) and high (80 dB SPL) signal level, a quiet and noisy listening environment, and no processing and processing by three different signal-processing algorithms of a hearing aid. All recordings were obtained using a Zwislocki coupler inside the Knowles Manikin for Acoustic Research. The purpose of this study was to determine whether the acoustic distance between confusable phonemes can be used to evaluate the performance of speech-processing algorithms used in nonlinear, digital hearing aids. The acoustic distance was the rms amplitude difference in the short-term spectra between any two speech segments after they were optimally aligned in time. It was hypothesized that: (1) There would be a significant reduction of acoustic distance among the selected phonemes when speech signals were filtered to simulate a given hearing loss configuration. (2) The use of a hearing aid would significantly alleviate the reduction of acoustic distance among these phonemes. Results, in general, were supportive to these hypotheses. It is, therefore, feasible to assess the information-processing function of a nonlinear, digital hearing aid using an instrument-based approach.

\section{$2: 20$}

4pEAa2. Clinical trials of a hybrid adaptive beamformer (HAB) for improved speech understanding in noise. G. L. Gibian, Walter Koroljow, Andy LaRow, Scott Shaw (Planning Systems, Inc., 7923 Jones Branch Dr., McLean, VA 22102), Peggy Nelson, and LaGuinn Sherlock (Univ. of Maryland School of Medicine, Baltimore, MD 21201)

A wearable, hybrid adaptive beamformer (HAB) device has been developed using a four-microphone array and a combination of adaptive and fixed-weight beamforming. The HAB is being evaluated with hard-ofhearing $(\mathrm{HoH})$ listeners in a variety of environments. Pilot tests used HINT test sentences and noise from separate loudspeakers (106-deg angle) in an audiological booth (AI-weighted direct-to-reverberant ratio $9.1 \mathrm{~dB}$ ). Measured sentence reception thresholds (SRTs) indicated that the HAB provided 14.5, 16.1, and $12.8 \mathrm{~dB}$ improvement over the single microphone for two normal-hearing and one $\mathrm{HoH}$ listener. Results will be reported for ongoing objective and subjective testing of elderly listeners with mild to moderate hearing losses. Subjects are fitted monaurally with a commercially available behind-the-ear hearing aid with a dual microphone array following the NAL-R fitting algorithm. Comparisons are made between listeners' SRTs in noise, using: (a) conventional single-microphone, (b) dual microphone, and (c) four-microphone HAB devices. HINT sentences are presented with single and dual noise sources at two arrival angles in three room environments: (a) sound booth, (b) favorable environment, and (c) noisy/reverberant environment. Listeners rate the three hearing aid systems and indicate their preference in paired comparison testing. SRTs, ratings, and preference judgments will be reported. [Work supported by NIH.]

\section{2:40}

4pEAa3. Qualitative and quantitative results from a speech enhancement scheme assessed by hearing-impaired subjects. P. W. Shields and D. R. Campbell (Dept. of Electron. Eng. and Phys., Univ. of Paisley, High St. Paisley, Renfrewshire PA1 2BE, Scotland, paul@diana22.pailsey.ac.uk)

A multi-microphone sub-band adaptive system for binaural preprocessing of speech signals for potential processing in future hearing aids has been investigated. Results are presented from both quantitative and qualitative assessments of the performance of the processing scheme as recorded from hearing-impaired subjects. The quantitative assessment is based on intelligibility testing of the algorithm using real-room recordings, $T_{60}=0.3 \mathrm{~s}$, with multi-talker babble as the unwanted noise source. This evaluation has been conducted by using the four-alternative auditory feature test developed by Foster and Haggard [J. Br. Audiol. 21, 165-174 (1987)]. A corresponding mean opinion score on speech quality from each test subject provides the qualitative analysis. A response time measure was included with the qualitative measure. The processing scheme uses the least mean squares (LMS) [S. D. Sterns and R. A. David, Signal Processing Algorithms (Prentice-Hall, Englewood Cliffs, NJ, 1988)] adaptive noise cancellation algorithm in frequency-limited subbands. The inputs from each microphone are split into 16 contiguous subbands using a cochlear distribution according the function provided by Greenwood [J. Acoust. Soc. Am. 87, 2592-2605 (1990)]. Each frequency-limited subband is processed using a LMS adaptive noise cancellation filter operating in an intermittent or continuous mode depending on input signal characteristics. The results show a statistically significant and practically valuable improvement in both intelligibility and perceived speech quality using the proposed scheme.

\section{3:00}

4pEAa4. Array technology for binaural hearing aid applications. Ivo L. D. M. Merks, Marinus M. Boone, and A. J. Berkhout (Lab. of Acoust. Imaging and Sound Control, Delft Univ. of Technol., Lorentzweg 1, 2628 CJ Delft, The Netherlands, ivo@akst.tn.tudelft.nl)

An increasing number of people have great difficulties in understanding speech in noisy environments. These difficulties can be resolved with a directional microphone array which attenuates the background noise while it transmits the desired signals unaltered to the hearing aid. A highly directional endfire array has been developed containing only four omnidirectional microphones which are integrated into the arm of a pair of spectacles. Two endfire arrays, one per ear, devise a binaural hearing aid which further increases the speech intelligibility and also enables localization. The arrays realize maximum directivity with a least-squares optimization of the array processing which also takes into account the diffraction effects due to the presence of the head. The microphone signals are pro- 
cessed with FIR filters to obtain maximum control over both the amplitude and phase of the transfer functions of the filters, thereby achieving maximum directivity. The microphone array attenuates the background noise of a diffuse sound field from $5 \mathrm{~dB}$ at $500 \mathrm{~Hz}$ to $10 \mathrm{~dB}$ at $4 \mathrm{kHz}$. The improvement in speech reception threshold (SRT) of 16 normal hearing and 26 hearing-impaired subjects will be measured and presented in this paper.

\section{3:20}

4pEAa5. Headphones as near-field electromagnetic radiators and probable health impairing objects. Florian M. Koenig (Ultrasone Electroacoustics GmbH, Schellenbergstrasse 7, D-82110 Germering, Germany,ultrasone@t-online.de)

The progress in electroacoustics offers a miniaturized, head-related sound source to produce spatial auditory events [F. M. Koenig, AES Preprint No. 4495 (1997)]. These objects, so-called headphones, are working as dynamic (electromagnetic) or electrostatic transducers. Irrespective of this exists knowledge by epidemic studies about low-frequency electromagnetic fields and their health-impairing effects on biological organisms worldwide. A well-known result is the "TCO ('95) environmental labeling of displays" or radiation reduced computer monitors and screens. Independent efforts point out that the TCO idea should be valid also for other electronical consumer products like halogen headlights, clock radios, or housing machines. Is it necessary to add the near-field reinforcing headphone? First measurements over 23 headphones gave the answer, Yes [F. M. Koenig, 24. DAGA (1998), in preparation]. Using a pink noise C-weighted signal by a usual SPL of $70 \mathrm{~dB}$ reveals that the majority of customary headphones generate an electromagnetic field emission at the human head, which crosses the border level of the TCO'95 or German recommendation for the housing area [VDE-DIN No. 0848, part 4A1/11/ 90]. Continued investigations underline this earlier result. The steps' contrary high-field dispositions will also be shown.

\title{
Session 4pEAb
}

\section{Engineering Acoustics and Noise: Implications of Recent Standards}

\author{
Victor Nedzelnitsky, Cochair \\ National Institute for Standards and Technology, Sound Building (233), Gaithersburg, Maryland 20899-0001, USA \\ Dieter P. A. Gottlob, Cochair \\ Federal Environmental Agency, Bismarckplatz, 1, D-14193 Berlin, Germany
}

Chair's Introduction-3:55

Invited Papers

4:00

4pEAb1. The role of international and European standardization in relation to European directives. Leif Nielsen (DS, ISO/TC43\&CEN/TC211 secr, Kollegievej 6, DK-2900 Charlottenlund, Denmark, len@ds.dk)

There are three different types of relations between standards and EU directives: (1) Self-contained directives: Basically such directives do not call for standards. However, though standards do not contain noise limits, many standardized measurement methods exist, and much work has been initiated in order to avoid conflicting measurement methods in directives and standards. (2) "Reference to standards:" Such directives may, e.g., contain noise limits, and measurement methods are given by direct reference to specific standards which may be international or European. (3) "New approach:" This is the most modern way of using standards in relation to directives. Such directives contain only so-called "essential requirements" leaving the technical content to be fulfilled by standards. Standards in this context are required by the EU Commission to be European standards prepared by CEN. The most wellknown example is the machinery safety directive. This has lead to the preparation of some 400 safety standards containing noise paragraphs. The EU commission has just started preparing a directive on external noise. Many standardization activities are also going on in this area. At the moment it is not known which type of directive is intended and which role the international and European standardization work will have.

4pEAb2. Strategy policy of ISO/TC43 “Acoustics.” Klaus Brinkmann (Physikalisch-Technische Bundesanstalt, Bundesallee 100, D-38116 Braunschweig, Germany, klaus.brinkmann@ptb.de)

The scope of ISO Technical Committee 43 "Acoustics," together with its Subcommittees "Noise" and "Building Acoustics" comprises standardization in the whole field of acoustics, i.e., the measurement of sound, its generation, transmission, and reception as well as its effects on Man and the environment. Close liaison is maintained with related international and regional standardization committees, especially IEC/TC 29, which is responsible for electroacoustic standards. ISO standards elaborated by TC 43 are equally addressed to a variety of parties, such as industry, regulatory authorities, trade and consumer groups, research institutes, testing laboratories and consulting engineers, health and safety inspectors, audiologists, and communication experts. Representatives of these groups from 24 different countries contribute actively to its work. More than 120 standards have been published so far and the present 
program of work includes nearly 80 projects for new or revised standards. In the past few years, major emphasis was laid on the measurement of machinery noise and the measurement and rating of sound insulation in buildings and of building products, both in the context of recent regional legislation. Fields like environmental noise, transport noise, noise prediction, and simplified measurement methods for building acoustics are considered to be future priority areas.

\section{4:40}

4pEAb3. New trends in standards indicate need for more interlaboratory comparisons and data. Victor Nedzelnitsky (Natl. Inst. of Standards and Technol. (NIST), Sound Bldg. 233, Rm. A147, Gaithersburg, MD 20899-0001, vnedzel@nist.gov)

Current trends in standards development now place, and will continue to place, significantly increased demands on laboratories around the world. Shorter IEC/ISO procedural deadlines and the increasing pace and complexity of standards development require that more work be accomplished in less time. Several trends account for the increasing complexity of measuring instrument standards from IEC TC 29 electroacoustics. One trend is the breadth and rapidity of technological developments in transducers and modern analog/ digital electronics. Other trends involve the inclusion in standards of more information regarding uncertainties, test methods, procedures, and EMC (electromagnetic compatibility). This information is needed because these IEC standards are being used to support development of OIML recommendations in legal metrology, as well as national and regional standards and regulations in many countries. The need to produce correct statements regarding measurement uncertainties, methods, and procedures greatly increases the need to acquire extensive, reliable data, especially from interlaboratory comparisons, both for the preparation of new standards, and for the necessary periodic revision of existing ones. Finding resources to meet this need is an important challenge for the field of acoustics. Activities begun in BIPM and other organizations will provide data for primary (but not necessarily secondary) acoustical metrology.

\section{5:00}

4pEAb4. The implementation of ISO/IEC guide 25 for acoustical standards at NRC Canada. George S. K. Wong and Lixue Wu (Acoustical Standards, Inst. for Natl. Measurement Standards, Natl. Res. Council, Ottawa, ON K1A 0R6, Canada)

Since the publication of ISO/IEC Guide 25, national acoustical measurement laboratories are under pressure to follow the recommendation of the Guide to update the calibration procedures to ensure confidence in the calibration services offered. The uses of the Guide (currently under revision as DIS 17025: General requirements for the competence of testing and calibration laboratories) promote cooperation between laboratories, acceptance of measured data in industries and in the harmonization of standards and procedures. In the international scene, the acceptance of calibration and test results between countries will ease the removal of nontariff barriers to trade. This presentation provides a brief discussion on the requirements and the strategy used to enhance the acoustical standards program at the National Research Council.

$5: 20$

4pEAb5. Hot new topics in ASA/ANSI standardization. Paul Schomer (Schomer \& Assoc., 2117 Robert Dr., Champaign, IL 61821)

The ASA Committee on Standards has many new work efforts underway. As chairman of the ANSI S12 committee (noise), this author is primarily versed in the hot new noise standards being developed. Hence, this paper will concentrate on noise standards. (The S12 committee can be considered the counterpart to ISO TC 43/SC 1 and concentrates on noise.) Several new efforts are underway in S-12, including (1) classroom acoustics, (2) room noise criteria, (3) model community noise ordinances, (4) community noise assessment, (5) consumer product noise labeling, and (6) in situ hearing protector measurements. In addition, there is a new effort in the S-3 committee (bioacoustics) dealing with warning signal definitions. Clearly, these new topics depart from many of the traditional standards topics such as measurement of workplace machinery noise or office and business equipment noise. Rather, these new work efforts concentrate on criteria development, measures to assess response to noise in various settings, or various human interactions with sound, etc. Several of these topics, such as community noise assessment, noise labeling, or hearing protector measurement, relate to ongoing international efforts. This paper will briefly describe the goals, progress, key contacts, and schedules for these work efforts.

\section{Contributed Paper}

\section{5:40}

4pEAb6. A simple three-dimensional (3-D) sound intensity probe. Erik Druyvesteyn, Hans-Elias de Bree (Univ. of Twente, P.O. Box 217, 7500 AE Enschede, The Netherlands, w.f.Druyvesteyn@el.utwente.nl), and Bert Roozen (Philips Res./CFT Labs., 5656 AA Eindhoven, The Netherlands)

An important property of sound intensity measurements is that the free-field properties of a sound source can be determined, although the source is positioned in a reverberant environment. The standard intensity probe consists of two closely spaced, accurately matched pressure microphones (p-p probe). A 3-D intensity probe consists of three pairs of matched microphones. The necessity of accurate matching makes such a probe large, complicated, and expensive. As an alternative for the p-p probe a $\mathrm{p}-\mathrm{u}$ probe has been published [104th AES Convention Amsterdam; preprint 4651], where the particle velocity $(u)$ is determined using the Microflown. It consists of two heated wires, $40 \mathrm{~mm}$ apart. The temperature difference of both wires is linearly dependent with the particle velocity $(u)$ and can be measured accurately. A 3-D intensity probe will be reported consisting of one (pressure) microphone and three particle velocity sensors placed orthogonal for the velocity vector $(u)$ determination. Experiments have been performed in an anechoic and a reverberation room. It was found that the free-field properties of a sound source, as determined from experimental results in the reverberation room, coincide with the experimental results in the anechoic room, showing that this 3-D intensity probe works satisfactorily. 
Session 4pMU

\title{
Musical Acoustics: Mapping Multiple Physical and Perceptual Attributes to Musical Structures
}

\author{
Roger A. Kendall, Cochair \\ Department of Systematic Musicology, UCLA, Box 951657, Los Angeles, California 90095-1657, USA \\ Stephen E. McAdams, Cochair \\ Laboratoire de Psychologie Experimentale (CNRS), 28 rue Serpente, F-75006 Paris, France
}

Chair's Introduction-1:55

Invited Papers

2:00

4pMU1. Timbral effect of parameter interchange between musical instrument types. James W. Beauchamp (School of Music and Dept. of Elec. and Comput. Eng., Univ. of Illinois at Urbana-Champaign, 2136 Music Bldg., 1114 W. Nevada, Urbana, IL 61801)

One method of studying the perceptual importances of physical parameters is to generate stimuli involving parameter interchange between instrument types. A new method of doing this models each instrument in terms of excitation and filter, derived from spectral analysis data, and includes independent temporal controls for fundamental frequency, spectral centroid, and amplitude. When temporal parameters are interchanged between instrument models, hybrid sounds result where it is possible to recognize perceptual aspects of both original instruments. When a parameter is inserted into a new environment, its value toward suggesting a particular instrument type may be enhanced. This paper explores conditions under which interchanged parameters may evoke particular instrument responses as opposed to simply merging with the new environment. Such salient parameters may assist in efforts to classify automatically instrument types.

4pMU2. Multidimensional scaling of musical timbre constrained by physical parameters. Stephen McAdams (Laboratoire de Psychologie Experimentale (CNRS), 28 rue Serpente, F-75006 Paris, France and IRCAM, 1 place Igor Stravinsky, F-75004 Paris, France, smc@ircam.fr) and Suzanne Winsberg (IRCAM, F-75004 Paris, France)

A new multidimensional scaling technique [S. Winsberg and G. De Soete, Br. J. Math. Stat. Psychol. 50, 55-72 (1997)] is applied to the analysis of dissimilarity judgments on musical timbres in both group and individual data. This technique constrains the resulting spatial model such that the order of items along a given perceptual dimension preserves their order along a previously established physical dimension. The fit between perceptual and physical dimensions is achieved with spline functions and yields what may be interpreted as the auditory transform of the physical dimension needed to obtain the perceptual one. A reanalysis of ten timbre spaces from the literature shows that this kind of model does not work as well on group data as it does on individual data due to differences in the nature of underlying dimensions and in the form of the auditory transforms for different listeners. Further, an analysis of individual data sheds light on the reasons why higher dimensions in published timbre spaces are so often difficult to interpret.

\section{2:40}

4pMU3. Multidimensional acoustical and psychoacoustical aspects of inharmonic plate and bar tones of real and resynthesized instruments. Edward C. Carterette and Roger A. Kendall (Music Percept. and Acoust. Lab., Dept. of Ethnomusicology, Univ. of California, Los Angeles, 405 Hilgard Ave., Los Angeles, CA 90095)

Following a brief review of the mathematics of inharmonic plates and bars, the acoustics and comparative psychoacoustics of the tones of Indonesian bars and plates are considered. Tones were derived from real instruments of the UCLA Department of Ethnomusicology's Javanese and Balinese gamelans, and from resynthesis of spectral analyses of instrumental tones. The comparative psychoacoustics of the tones was assessed by a number of methods, including JNDs, ratings by MDS similarity-scaling, and verbalattribute magnitude estimation. Several methods were used to converge on the general perceptual and cognitive properties of the tones. Illustrative findings are: It was shown (1) that Indonesian gamelan makers can and do tune their metallophones in stretched octaves. Their stretchings (1.02 to 1.05 times the normal octave) were fit by a mathematical model which was derived from the data of many gamelans. (2) That the perceptual dimensions of Indonesian and Balinese gongs were interpreted as pitch, ombak (beating), and volume. These dimensions can be mapped to frequency, amplitude modulation, and spectral envelope. It was concluded that gong makers can modify the plates to achieve desired physical properties and correlated perceptual dimensions. One important finding is that the relative positions of two close axially symmetrical modes control the ombak rate. 
4pMU4. Memory for melodies, poetry, faces, and other complicated things. W. Jay Dowling (Univ. of Texas at Dallas, Richardson, TX 75083-0688)

For over 100 years it has been a well-known "fact" of research on memory that after something is memorized, it is gradually forgotten over time. This is largely true of the relatively impoverished and meaningless materials that memory researchers have typically relied upon. As early as 1913, Ballard showed that poetry can be recalled better and better following memorization ("hypermnesia"), and recent work by Erdelyi and others supports those results. Hypermnesia in recognition, however, has proved elusive. The present experiments demonstrate hypermnesia in recognition memory for unfamiliar folktunes. The complexity and meaningfulness of stimuli such as melodies, poetry, and perhaps faces evoke memory processes that lead to improvement rather than decay over the early span of time following acquisition.

3:20

4pMU5. What makes singing expressive? Johan Sundberg (Voice Res. Ctr., Speech Music Hearing, KTH Stockholm, Sweden)

Answers to this question are sought in two experiments analyzing performances of songs from the classical Lieder repertoire as sung by professional baritone singers. In one experiment, two versions of the same excerpts were compared; in one the singer attempted first to sing with as little expression as possible, and then as in a concert. In another experiment, a professional singer performed a Lied with a prerecorded piano accompaniment provided over earphones (a) with the original text of the poem and (b) replacing the text syllables with the syllable [mV:]. The significance to expressiveness of observed differences is evaluated by synthesis experiments. The results suggest that expressivity contains information on musical structure, facilitates categorization of tones, and adds an emotional coloring. Expressivity is communicated by means of a code involving meaningful modulation of various parameters such as tempo, pitch, loudness, and vibrato extent. It also includes certain characteristic patterns such as ascending glides to target pitches, and smoothing or sharpening of loudness and pitch contours.

\section{3:40-4:00 Break}

\section{4:00}

4pMU6. Vibrato and portamento, hypotheses and tests. Peter Desain (Music Mind Machine Project, NICI/KUN, Nijmegen, The Netherlands)

As Seashore already pointed out, continuous aspects in music performance, which happen during and in between notes, can be a relevant means for expression. These modulations, like vibrato and portamento, are very consistently controlled and seem to exhibit striking regularities. However, there are many conflicting hypotheses in the literature about these regularities, especially concerning the relation between vibrato and global tempo. To investigate these more systematically, experiments were conducted in which different instruments perform the same piece at different tempi. The results, relating vibrato rate, extent, and phase to tempo and timing will be presented. Apart from understanding the process of music performance better, they may be applied in the design of synthesizers and software for the music studio.

4:20

4pMU7. Acoustical specification and musical meaning. Eric F. Clarke (Univ. of Sheffield, Sheffield S10 2TN, UK)

Musical meaning has commonly been regarded as only very distantly related to music's acoustical surface. This paper proposes that there is actually a rather close relationship between the two-that musical meaning is specified with considerable immediacy in sound. An important aspect of the meaning of an everyday sound in the environment is its source, and this same principle can be interestingly extended to music if the notion of "source" is generalized somewhat to encompass more than just physical/instrumental origin. Following a brief introduction to the theory of ecological acoustics on which such a claim is based, and an illustration of its application to everyday environmental sounds, a number of musical examples will be used to illustrate the diverse aspects of musical meaning that are specified in musical sound, ranging from states of the performing body to the cultural allegiances of musical genres.

4:40

4pMU8. Cross-modal integration: Synchronization of auditory and visual components in simple and complex media. Scott D. Lipscomb (Inst. for Music Res., Div. of Music, Univ. of Texas, 6900 N. Loop 1604 West, San Antonio, TX 78249)

Recently, there has been a significant amount of interest in the perceptual interaction between auditory and visual (A-V) systems in multimodal contexts. Both psychologists and musicians are beginning to investigate the manner in which a stimulus perceived in one sensory modality may affect the cognitive processing of a stimulus in a separate modality. Beginning in the 1950s, a series of psychophysical investigations revealed cross-modal influences using extremely simple stimuli. Most studies incorporating more complex stimuli have focused on the referential aspect of musical sound, i.e., the "cognitive congruency" of the music and the visual images. The present study will specifically investigate the alignment of accents (i.e., salient events) in the auditory domain with those in the visual domain, and the effect of this alignment on subject perception of the $\mathrm{A}-\mathrm{V}$ composite. The author will report on three experiments utilizing varying levels of stimulus complexity: single-object animations, animations by Norman McLaren, and motion picture excerpts. A-V alignment was manipulated as the independent variable with subject ratings of effectiveness as the dependent variable. A revised model of film music perception will be presented, proposing a dynamic relationship between stimulus complexity and significance of AV synchronization in the determination of subject ratings. 
4pMU9. Music serves as a vehicle in multimedia contexts. Annabel J. Cohen (Dept. of Psych., Univ. of Prince Edward Island, Charlottetown, PE C1A 4P3, Canada)

The vast amount of information in multimedia presentations ought to place inordinate demands on perceptual and cognitive systems. Yet there seems to be a superfluity of resources for processing music in the midst of processing information from visual and verbal sources. Research of the author and others reveals the remarkable ability of listeners to accomplish three kinds of musical processing in multimedia contexts: picking up cross-modal structural similarities, generating meanings and associations, and establishing cross-modal linkages in memory. Emphasizing principles of structural congruence and semantic association [S. E. Marshall and A. J. Cohen, Music Perc. 6, 95-112 (1988)], a framework is described for examining these musical processes in the context of simultaneous visual and verbal input streams [A. J. Cohen, Proc. 5th International Conf. on Music Perc. and Cog. (1998), pp. 13-20]. The present paper highlights how music transports different types of information (e.g., acoustic, temporal-structural, semantic). These different types of information carried by the music can be selectively exploited to accomplish such functions as masking, focusing attention, disambiguation, reminiscence, suspension of disbelief, and creation of aesthetic experience. [Work supported by SSHRC.]

\section{$5: 20$}

4pMU10. Identification and qualification of instrumental sound sources: Elaboration. Catherine Sémidor, Léonie Couthon, and Aline Barlet (ERIAC Ecole d'architecture et de paysage de Bordeaux, Domaine de Raba, 33405 Talence Cedex, France, catherine.semidor@bordeaux.archi.fr)

From multitrack anechoic recordings of orchestral, or other musical instruments, a quantitative analysis of musical instruments' power and directivity was conducted [Semidor and Couthon, ICA98]. A qualitative study is now proposed to complete the knowledge about the perceived sound of these same instruments by listeners. The following elements are examined: (1) the "recognizing threshold" of instrumental sources, from a directivity point of view. Particularly, studying the differentiation difference between sources of the same family, or sources with a pronounced directivity and others. (2) The subjective "qualification" of sources in function of directivity, that is the influence of the quantity of information on directivity, on the qualification of the source. (3) Correlation of those results with the quantitative study conducted before. Are the main objective parameters noticed in a subjective point of view? This study is conducted in a first approach in laboratory conditions. Its main applications are sound recording, sound reinforcement, measurement, and simulation in room acoustics. It is well known that taking into account sound sources' characteristics permits the improvement of a room's design in order to increase the audience comfort.

\section{Contributed Papers}

$5: 40$

4pMU11. Amplification of the difference tones $(f 2-f 1)$. Pierre Dutilleux (ZKM|Inst. for Music and Acoust., Karlsruhe, Germany)

Difference tones have been known by composers and performers for a long time. Nevertheless, these tones are still difficult to use in a musical context because their appearance is fairly unpredictable. A cello player, Michael Bach, has developed a sophisticated playing technique to produce these tones. Unfortunately the public was not aware of it because it could not here them as well as the player did. Evidence of the presence of the difference tones in the acoustic signal has been found in some cases. In most cases these tones develop in the head of the listeners only at fairly high sound-pressure levels. In order to control the amount of difference tones in a musical sound, a processing method has been developed that artificially produces the difference tones. The squared and filtered instantaneous envelope of the acoustic signal delivers them. These tones can then be mixed to the original sounds as needed and the public can perceive the difference tones even at moderate sound-pressure levels. The system was implemented and performed well during concerts. With a celletto (cello without any sounding box) it is possible to hear only difference tones.
6:00

4pMU12. Parsing complex rhythmic structures: The contribution of spectral and temporal dimensions of timbre. Punita G. Singh (Sound Sense Consultancy Services, 20-A Aurangzeb Rd., New Delhi 110011, India, pgsingh@hotmail.com)

Perceptual parsing of sequences with multiple possible metric interpretations was studied. Sequences of 12 complex tones were used as stimuli, with either no deliberate accents provided, or physical accents introduced by changing timbre at positions implying a triple, quadruple, or multiple meter (i.e., both triple and quadruple simultaneously). Subjects reported if they perceived a triple meter, quadruple meter, ambiguous meter, or no meter. The number of harmonics $(2,4$, or 8$)$ or the locus of three harmonics were the spectral variables used to mark timbre accents. Steepness of rise and decay time $(95+5 \mathrm{~ms}$ versus $5+95 \mathrm{~ms})$ was the temporal variable used. These attributes served well as accent markers for sequences with unambiguous meters. However, listeners were generally unable to parse sequences where multiple meters were provided by the same timbre cue. For sequences where multiple meters were provided by contrasting timbres, listeners generally picked the metrical structure implied by spectrally richer or brighter timbres comprising more or higher harmonics. Temporal envelope slope was not effective in facilitating parsing of meters. 


\title{
Session 4pNSa
}

\author{
Noise: Military Aircraft Noise \\ Bernard F. Berry, Cochair \\ National Physical Laboratory, Teddington TW11 OLW, UK \\ Lawrence S. Finegold, Cochair \\ U.S. Air Force Research Laboratory, Wright-Patterson AFB, Ohio 45433, USA \\ Chair's Introduction-1:55 \\ Invited Papers
}

2:00

4pNSa1. A review of recent military aviation noise programs. Eric Stusnick (Wyle Labs., 2001 Jefferson Davis Hwy., Ste. 701, Arlington, VA 22202, estusnic@arl.wylelabs.com)

This presentation reviews recent studies that have been carried out by Wyle Laboratories on noise from military aircraft operations. Four different areas of research will be described—modeling, measurements, psychoacoustics, and noise control. The discussion of subsonic aircraft noise models includes NOISEMAP, ROUTEMAP, MR_NMAP, and the Rotorcraft Noise Model, while that of supersonic noise models includes CORBOOM, BOOMAP3, and PCBOOM. The discussion of aircraft noise measurements includes sonic boom monitoring, military training route measurements, NOISENET, potential damage to unconventional structures by sonic boom, and rotorcraft noise measurements. The psychoacoustic discussion includes the development of the onset rate correction for high-speed, low-flying military jet overflights and community response to sonic booms. The discussion of aircraft noise control will describe the use of active noise control to reduce noise from hush houses and static jet engine runups.

\section{2:20}

4pNSa2. U.S. Air Force research on military aircraft noise and its effects. Lawrence S. Finegold, Robert A. Lee, and Richard L. McKinley (U.S. Air Force Res. Lab., Wright-Patterson AFB, OH 45433, LFinegold@falcon.al.wpafb.af.mil)

For over half a decade, the U.S. Air Force (USAF) has been conducting a program of research on military aircraft noise and its effects. This paper provides a brief overview of some of the major projects implemented at Wright-Patterson Air Force Base in the past decade on noise measurement and monitoring, noise propagation modeling, and the effects of noise on humans, animals, and structures. It also includes both sonic boom research and subsonic aircraft overflight noise. Several international collaborative efforts have been conducted with NATO partners on both fixed-wing aircraft and helicopter overflight noise. In addition to the community noise portion of this program, the USAF has also conducted research on the auditory effects of military occupational noise exposure, development of hearing protection devices, and the development of three-dimensional audio information presentation. Although time limits preclude a comprehensive review of the extensive USAF program, highlights of some of the most interesting and important projects will be provided.

\section{2:40}

4pNSa3. Adjustment factors for assessing long-term aircraft and other noise environments. Paul Schomer (Schomer \& Assoc., 2117 Robert Dr., Champaign, IL 61821)

LEQ and DNL are commonly used to assess the long-term noise environments around airports, roads, etc. Frequently, the measure used is the long-term (e.g., yearly average) DNL or long-term daytime LEQ, etc. Schultz and others have related the long-term DNL or LEQ to community response as measured by the percent of a community that is highly annoyed. These relations exhibit a large scatter to the data. It is possible for a specific community to exhibit many times the percent highly annoyed predicted by these "standard" relationships. Some have suggested adjustments to LEQ or DNL to better explain community response and reduce the scatter in the relations between DNL and percent highly annoyed. These adjustments include "busy day," a "nave" community (not familiar with the sound), a "quiet rural setting," and the presence of "rattles." This paper uses experimental data, case histories, and logic, to examine the efficacy of these adjustments.

3:00

4pNSa4. Modeling of military aircraft noise in support of environmental impact mitigation. Neil M. Standen (Jacques Whitford Environment Ltd., 2781 Lancaster Rd., Ottawa, ON K1B 1A7, Canada) and Gary W. Humphries (Dept. of Natl. Defence, Ottawa, ON K1A 0K2, Canada)

The Military Training Area in Labrador, Canada is used by NATO Alliance air forces of the United Kingdom, Germany, and the Netherlands, and is managed by the Canadian Department of National Defence (DND). Protection of the natural environment is a major consideration in the management of the training area. This paper describes a computer-based noise propagation model that will be used to support environmental management. The paper demonstrates its use in planning flying training sorties to mitigate short- 
term environmental impacts, and also its use in assessing environmental effects of the flying activity over the longer term. The model enables DND to maximize the extent of the range available for flight training by accurately calculating noise levels on the ground, and hence the degree and method of noise impact mitigation that is appropriate. The noise propagation model is used in conjunction with other computer-based tools for flight track modeling, terrain modeling, and specifying locations of noise-sensitive areas (typically wildlife habitats which may change in location during the training period). Other projects are aimed at investigating and evaluating acceptable levels of noise impact, in terms of consequences on wildlife viability in these habitats.

4pNSa5. The theoretical and practical aspects of producing source data for helicopter noise modeling. R. J. Weston and R. Beaman (RAF Inst. of Health, RAF Halton, Aylesbury, Bucks HP22 5P6 UK)

Accurate source data are the key to future developments in noise modeling. With the increasing need to model helicopter noise, suitable source data will be required. For helicopters, the directivity of the noise source is an essential component of the model. This paper reviews a system for determining helicopter directivity together with a discussion of future developments. An overview of an international helicopter noise measurement trial conducted in June 1998 to determine directivity will be presented along with some of the data.

\section{3:40-4:00 Break}

4:00

4pNSa6. Military aircraft noise and health-Methodological issues in research. Ian H. Flindell (Inst. of Sound and Vib. Res., Univ. of Southampton, Southampton SO17 1BJ, UK)

The possibility of long-term health effects associated with prolonged exposure to military low-altitude flying (MLAF) noise in designated training areas in the UK remains an area of public concern. Definitive research in this area is difficult for many reasons. In addition to the general problems which apply to all noise and health research; of defining the effects of variables of interest; of estimating individual exposure; of independently identifying susceptible individuals; and of controlling for confounding factors, the generally low levels of MLAF noise exposure (measured as outdoors LAeq) and the wide variability of training schedules to meet varying tactical requirements create further difficulties. MLAF events are often irregular, infrequent, and may have higher maximum levels and higher onset rates than other types of environmental noise. What are the theoretical reasons why these factors should contribute to increased risks of health effects? Acoustic startle effects and associated physiological responses can equally be interpreted either as signs of healthy reactivity or as precursors of disease. MLAF events can contribute to acute annoyance at the time that they occur, but the possible mechanisms for noise-related annoyance to contribute to longer term morbidity through some vague and unspecified stress hypothesis are obscure.

4pNSa7. The response of the acoustic reflex to noise from low-flying military aircraft. Geoff Kerry, Claire Lomax, Peter D. Wheeler (Univ. of Salford, Salford M5 4WT, UK), and David J. James (Royal Air Force Innsworth, Gloucester GL3 1EZ, UK)

Noise from low-flying military jet aircraft is often characterized as loud and sudden. Some researchers have suggested that the rise time of noise from such operations might be faster than the response time of the acoustic reflex, reducing protection to the inner ear. Recordings of Tornado GR1 overflights have been applied to simple models of the middle ear taken from the literature to estimate the likely response of the acoustic reflex and the attenuation of noise transmitted to the inner ear. Results indicate the reflex will provide some degree of protection to this type of noise, and that the rate of rise of the stimulus influences both the speed of the reflex response and the attenuation at a specific time. This procedure is complicated and relies on certain assumptions being made. Another approach is considered which uses complex computer models of the human ear, which allow simulation of a range of conditions together with the possibility of defining the cochlear response. Both approaches are presented, and the direction of future work toward defining a descriptor for the assessment of possible noise-induced auditory effects is discussed.

4pNSa8. Military aircraft jet overflights and children's hearing_Allegations, issues, and measurement uncertainties. Peter D. Wheeler and Nikki Matkovits (Univ. of Salford, Salford M5 4WT, UK)

The scientific literature of the past 20 years contains several references to the use of high-frequency audiometry for investigating susceptibility to, and early diagnosis of, noise-induced hearing loss. More recently, the measurement of high-frequency hearing acuity in children has been cited as an important tool in the identification and attribution of noise-induced hearing loss from alleged exposure to transient noise sources such as low-flying military jet aircraft. The enormous range of hearing thresholds found above $8 \mathrm{kHz}$, even in young adults, has been reported by many researchers. Measurement uncertainties in the range $8-16 \mathrm{kHz}$ can be substantial, and detection judgments at such frequencies for inexperienced subjects represent a further source of variance. This study aims to quantify the uncertainties associated with high-frequency audiometric measurements on children and adults in order to inform the interprtation of existing data and assist in the design of new studies. The outcome will be reported and taken into account in a review of the conclusions of recent published work involving high-frequency audiometry for the measurement of children's hearing in areas subjected to low-altitude military jet overflights. 
4pNSa9. Presenting noise information to community decision-makers. David T. Dubbink (Noise Management Inst., 864 Osos St., Ste. D, San Luis Obispo, CA 93401)

Resolving community noise problems requires not only accurate data about noise impacts, but also demands an effective means of communicating this information to decision-makers. The Interactive Sound Information System (ISIS) is a "new media" package designed to explain and demystify the complex metrics used to describe noise impacts. Central to the ISIS package is the idea that "real" noise examples, shaped to reflect local situations, are the very best way to build an understanding of noise management issues. The system has been used effectively by governmental agencies and noise management specialists as a means of delivering information that is both understandable and technically accurate. The United States Air Force, in association with the FAA, has supported development of an expanded version of the program that incorporates state-of-the-art multimedia technology. The new version of the program is designed for a wide audience, and its operation does not demand technical expertise. It uses the 3-D viewing capabilities of the Virtual Reality Modeling Language (VRML). The updated software is built on the Java platform, giving the package both modularity and Internet capabilities. The program links to airport and highway noise prediction models.

THURSDAY AFTERNOON, 18 MARCH 1999

ROOM H1028, 1:55 TO 5:40 P.M.

\title{
Session 4pNSb
}

\section{Noise and Psychological and Physiological Acoustics: Product Sound Quality}

\author{
Dennis Walton, Cochair \\ Abbott Laboratories, P.O. Box 152020, Irving, Texas 75015-2020, USA \\ Armin Kohlrausch, Cochair \\ IPO Center for Research on User-System Interaction, P.O. Box 513, NL-5600 MB, Eindhoven, The Netherlands
}

\author{
Chair's Introduction-1:55 \\ Invited Papers
}

4pNSb1. The perception of product sound quality. Ute Jekosch (Inst. of Commun. Acoust., Ruhr Universität Bochum, D-44780 Bochum, Germany, jekosch@ika.ruhr-uni-bochum.de)

There are different methods and methodologies available to assess the quality of sounds. Generally, instrumental and auditory assessment can be distinguished. For instrumental assessment, physical data are collected and analyzed, and a sound quality value is computed in the end. Auditory assessment methods use human listeners who judge on the quality of a perceived sound. Experts very often face the fact that instrumental and auditory quality values are not in accordance with each other. This holds especially for the case where instrumental methods are used to predict how a human listener will perceive the quality of a sound. It is the task of sound quality assessment research to abridge the gap between predicted and experienced sound quality. There are different approaches to reach this goal. The talk concentrates on one specific aspect, namely on the function of sounds. Sounds convey a meaning. The meaning is assigned to them by the listener. The relation between form and content is either arbitrary or fixed. Instrumental and auditory sound quality assessment have to consider these aspects: An appropriate assessment of sound quality has to include the aspects of the acoustic/auditory form, the listener, and the meaning he/she assigns to the auditory event.

4pNSb2. Development of a noise criterion for laboratory medical instrumentation. William H. Muto (Abbott Labs., P.O. Box 152020, Irving, TX 75015-2020)

Over the past two decades, medical laboratory instrumentation has undergone a major transformation in terms of its functionality, sophistication, and degree of automation. A common complaint among laboratory professionals using these instruments has been that laboratory noise levels have increased, and that certain instruments are especially noisy. The goal for this project was to establish an instrument noise criterion that would serve as a standard for the company's future generations of instruments. The ideal standard in 
this context would eliminate or greatly reduce customer complaints due to noise without significantly increasing development and manufacturing costs. After surveying various noise criteria and after preliminary investigatory studies, a variation of balanced noised criteria curves (NCB), proposed by Beranek (1989), was adopted as the basis for the standard. The current presentation will provide: (1) a description of the adopted noise criteria, (2) practical considerations for use in the current context, (3) measurement methods and tools used, and (4) a summary of progress to date regarding the use and utility of the adopted noise criteria.

2:40

4pNSb3. Adequacy of product sound: Design-develop test; is it so simple? Michiel A. A. Schallig (Oliemolenstraat 5, Drachten, The Netherlands)

Philips DAP B.V. is a major producer of consumer household appliances. Electric shavers and vacuum cleaners are examples of the products that are produced. Consumer satisfaction is of paramount importance for the company. This is obvious for the functional aspects of the appliance, but satisfaction goes beyond functionality. Design, price, ergonomics, and sound are a few aspects which can contribute to the desired level of satisfaction. To achieve this delicate balance within such different constraints is a difficult process. Control of the sound production of appliances is something that has to be tackled from the start of the development, but becomes increasingly difficult. Especially "softer" constraints like "sound quality" blurr the development process. Despite the difficulties, these softer aspects are considered more and more important. In the real development processes, however, it is, for example, very difficult to get good measurement data of human perception. Often this is done by panel research, but this is a very slow process and depends heavily on the availability of the correct panels. This is one of the many problem aspects that are related to developing appliances with a "quality sound." Some real world experiences, observations, and problems on the road to adequate sounds are described.

\section{3:00}

4pNSb4. Combined application of computer simulation and sound quality in the design of a home appliance. Brandon D. Tinianow (Johns Manville Tech. Ctr., 10100 West Ute Ave., Littleton, CO 80127, tinianow@jm.com) and Benjamin Cimerman (Vibro-Acoust. Sci., Inc., Los Angeles, CA 90010)

Computer-generated simulation tools are commonly used for acoustical product design, but often for spectral levels only. Sound quality, as expressed in sones and phons, quantifies sound as perceived psychologically by a listener, but possesses no predictive capability. In this study, both methods were combined to improve an appliance's performance in terms of noise. Using intensity mapping, noise paths contributing most to user annoyance were identified. A computer model of the appliance was then created using the widely recognized statistical energy analysis method. This model was employed to simulate design modifications targeting the paths identified as most critical by the sound quality study. Design modifications were implemented in a hardware prototype of the appliance as suggested by the model and this re-designed appliance was again subjected to the sound quality assessment. Results show that the appliance's performance was significantly improved.

\section{Contributed Papers}

\section{$3: 20$}

4pNSb5. Three-dimensional localization and characterization of acoustical sources in a truck cabin. Patrick Dubail and Manell E. Zakharia (CPE Lyon, LASSSO (LISA, EP92 CNRS), 43 Bd. du 11 Novembre 1918, BP 2077, Bat. 308, F 69616, Villeurbanne Cedex, France)

In the framework of acoustic comfort, an accurate localization (in range and angles) of the sources transmitting noise to the driver was investigated. The aim of such a localization is to act on the predominant sources in priority (in a given frequency range). The sources were either vibrating ones corresponding to the excitation point, or fictive ones corresponding to complex propagation and multiple reflections on the cabin elements. An experiment has been conducted on a real cabin with a single point of excitation. The mechanical excitation was achieved via a shaker; frequency-modulated signals have been used that cover a very wide frequency range $(0.03$ to $15 \mathrm{kHz})$. Both active and digital corrections were used. Two crossed line arrays of 112 microphones were used for the reception, all the signals were digitized and processed later on. Standard wideband beamforming and beam steering were used on both arrays (in the time domain). For every frequency (or frequency range), the 3D localization (horizontal and vertical angle, time) of sources was achieved. The major sources (15 to 20 ) were isolated using the radon transform. A fine analysis was achieved in order to provide, in addition to their 3-D localization, their spectral content.

\section{3:40}

4pNSb6. Exploration of associated imaginations on sound perception (AISP): A method for helping people to describe and to evaluate their sound perceptions. Petra Muckel, Leo Ensel, and Brigitte SchulteFortkamp (Dept. of Psych. (A6), Univ. of Oldenburg, D-26 111 Oldenburg, Germany)

A qualitative method especially developed for helping people to describe and evaluate their sound perceptions will be presented: The development of this method for "Exploration of Associated Imaginations on Sound Perception" (AISP) took place in a project about interior car sounds and possesses relationships with CIS (categorical scaling intermittent and subsequent thinking aloud) by Schulte-Fortkamp. Its starting points are the subjects' spontaneous imaginations and memories when they are going to listen. Focused on the effects of sounds, the design of AISP encourages people to describe their perception of sounds by telling their spontaneous associations, e.g., imaginations, memories, and embedded sensations and feelings in their own words and fashion. In an interview aided by a questionnaire, the subject evaluates sounds in leading his/her attention to his/her own imaginations, memories, and feelings while listening. These imaginations function as a context and biographical background for the subject's feelings; they make it easier to find words for the description of the sound perception, especially for the emotional effects of sounds. The purpose is to learn about descriptions of sounds and their possible emotional effects by associated imaginations and feelings. 


\section{4:20}

4pNSb7. A semantic differential design especially developed for the evaluation of interior car sounds. Nicolas Chouard (Dept. of Acoust., Univ. of Oldenburg, D-26111 Oldenburg, Germany, nicolas@aku.physik.uni-oldenburg.de) and Thomas Hempel (Univ. of Bochum, D-44780 Bochum, Germany)

The use of semantic-differential (SD) procedure allows sound designers to compare interior car sounds on the basis of polarity profiles. In order to get representative information, SD designs have to be based on carsound-specific verbal spaces. Due to the peculiarities of languages and conceptions of car culture in different countries, it is, moreover, not sufficient to use translated lists as they are available in the literature. In this contribution, the development of a representative verbal space for German subjects is presented. Two different approaches are used which are finally combined in order to get an intermediate list of adjective pairs. A representative and usable set of adjective pairs is established by reducing this list on the basis of SD tests with car sounds presented at the same loudness. The different steps and drawbacks in the development of the SD design are described and discussed. The components as well as the underlying dimensions of the verbal space are presented.

\section{4:40}

4pNSb8. A system for natural reproduction of sound and vibration. Hermann Remmers (ITAP GmbH, Carl-von-Ossietzky Str. 9-11, D-26129 Oldenburg, Germany, remmers@itap.de), Carsten Reckhardt, and Michael Bellmann (Univ. of Oldenburg, D-26111 Oldenburg, Germany)

For the psychoacoustical evaluation of sound, usually artificial head recordings are reproduced with headphones or loudspeakers. Interior aircraft or car noise, however, contains high sound and vibration energy components below $20 \mathrm{~Hz}$. If these spectral components are not reproduced, subjects often complain about an unnatural hearing impression. In this paper, a reproduction system is presented that extends the audio bandwidth down to $2 \mathrm{~Hz}$. For presenting vibration signals, the subject's chair (e.g., aircraft or car seat) is mounted on an exciter system. The threedimensional vibration signal spans the frequency range from 6 to $80 \mathrm{~Hz}$. A report is given on psychoacoustic experiments and results with this sound and vibration reproduction system.
4pNSb9. Affective judgment of aircraft sound quality. Daniel Västfjäll (Chalmers Rm. Acoust. Group, Dept. of Appl. Acoust., Chalmers Univ. of Technol., SE-412 96 Göteborg, Sweden), Àrni Ingvarsson (Saab AB, SE-581 88 Linköping, Sweden), and Mendel Kleiner (Chalmers Univ. of Technol., SE-412 96 Göteborg, Sweden)

Emotion is an important feature of the description and evaluation of sound quality, but this fact has often been treated as a "nuisance variable", in sound quality. The objective of this study was to investigate sound quality descriptors of aircraft sounds. The approach to descriptors in this case originated from the need for consideration of emotion in sound quality research. Thirty-two participants $(n=32)$ rated eight recorded sounds from turboprop aircraft binaurally replayed over headphones. Subjects rated 21 descriptors designed to capture the subjective dimensions of aircraft sounds. The descriptors were commonly used sound quality adjectives, which have emotional/affective connotation. The principal component analysis resulted in two main components. The first factor was interpreted as pleasantness and the second factor as stimuli intensity. These results indicate that affective descriptors can model perception of aircraft sound, i.e., emotional evaluation is important to aircraft sound quality. This study indicates that both description and judgment/decisions about sounds can be based on emotions. One approach to understand and improve sound quality modeling is to investigate the subjective emotional impression of sounds. [Work supported by NFFP Grant No. 232.]

\section{$5: 20$}

4pNSb10. Sound quality design for high-speed trains' indoor noise: Psychoacoustic evaluation of tonal components. Christine Huth, Hugo Fastl (Inst. of Man-Machine-Commun., Tech. Univ. München, Arcisstr. 21, D-80333 München, Germany), Ulrich Widmann (Müller BBM, Planegg), and Georg Hölzl (Deutsch Bahn AG, BT 512)

For the specification of the sound quality inside future high-speed trains, tonal components produced by the motors or corrugated rails can play an important role. Therefore, in psychoacoustic experiments, the dominance of tonal components at 630 or $1250 \mathrm{~Hz}$ was assessed. For an increase of the corresponding $\frac{1}{3}$-oct band by $20 \mathrm{~dB}$, a clear tonal character is audible which is only half as pronounced for an increase of $12.5 \mathrm{~dB}$ at $630 \mathrm{~Hz}$ or $10 \mathrm{~dB}$ at $1250 \mathrm{~Hz}$. In line with the expectation, no tonal quality is perceived, if the $\frac{1}{3}$-oct band in question is not enhanced. However, a decrease of sound energy in a $\frac{1}{3}$-oct band by $20 \mathrm{~dB}$ can also produce a faint tonal sensation with a magnitude of about $\frac{1}{10}$ of the tonal sensation produced by an increase of $20 \mathrm{~dB}$. The results obtained with stimuli simulating the sound quality inside high-speed trains are in good agreement with data from basic psychoacoustic experiments. Therefore, it is expected that sound quality evaluation of high-speed trains indoor noise can profit from a multitude of psychoacoustic data available.

\section{Contributed Posters}

These posters will be on display in the Poster Gallery from Thursday to Friday, 18-19 March. Authors will be at their posters from 10:00 a.m. to 12:00 noon on Thursday, 18 March.

\begin{abstract}
4pNSb11. Evaluation of interior car sound with a new specific semantic differential design. Thomas Hempel (Inst. of Commun. Acoust., Univ. of Bochum, D-44780 Bochum, Germany, hempel@ika.ruhr-uni-bochum.de) and Nicolas Chouard (Univ. of Oldenburg, D-26111 Oldenburg, Germany)
\end{abstract}

In an accompanying contribution [see Chouard and Hempel, "A semantic differential design especially developed for the evaluation of interior car sounds"'] the development of a semantic differential design based on a verbal space adapted to the description of interior car sounds was presented. In this contribution, this is used in semantic differential tests in order to characterize the interior car sound of eight different cars presented at the same loudness. Results of polarity profiles as well as factor analysis and cluster analysis on the set of cars are presented. Advantages and drawbacks of the method are discussed from the point of view of the experimenter.

4pNSb12. Motion perception and loudness judgments. Andreas Hellmann (Univ. Oldenburg, Dept. of Psych., D-26111 Oldenburg, Germany, hellmann@psychologie.uni-oldenburg.de)

Besides its acoustic properties, many other nonacoustic properties have been shown to influence the judgments of loudness, noisiness, and noise effects of sounding events. In the environment, one finds moving as well as nonmoving sound sources. The sound emitted may be either constant or dynamic. In an experiment, the effect of the perceived motion of 
a sound source or sound event on loudness judgments was investigated. Sounds were presented which were ambiguous and could be perceived both as moving and as nonmoving events. In different experimental conditions, the same sound stimuli were presented and introduced as moving or nonmoving events. The subjects judged the loudness of the events. At the end of the experiment, it was reported whether they had perceived the sound events as moving, nonmoving, or both. The results show an effect of perceived motion on judged loudness: Loudness judgments are higher if the sound events are perceived as moving than if they are perceived as nonmoving.

THURSDAY AFTERNOON, 18 MARCH 1999

ROOM H2013, 1:55 TO 6:20 P.M.

\title{
Session 4pNSc
}

\section{Noise: Urban Acoustics}

\author{
Jean-Dominique Polack, Cochair \\ Laboratoire d'Acoustique Musicale, University Paris VI, Case 161, 75252 Paris, Cedex 05, France
}

Roger Wayson, Cochair

Civil and Environmental Engineering Department, University of Central Florida, P.O. Box 162450, Orlando,

Florida 32816, USA

Chair's Introduction-1:55

\section{Invited Papers}

\section{2:00}

4pNSc1. A cognitive approach of urban soundscapes. Daniele Dubois and Sophie David (LCPE, ENS, 1, rue Maurice Arnoux, 92120 Montrouge, France, dubois@idf.ext.jussieu.fr)

The presented cognitive approach of noises in urban environments focuses on meaning and as such integrates linguistic, psychological, and acoustic conceptualizations and methodologies. It deals with the acoustic phenomena that are perceived, conceived, and said as relevant by the subjects. The productivity of this approach has been evaluated in two research programs. Experiments combining linguistic and psychological analyses showed that urban soundscapes include a complex combination of unpleasant and pleasant noises. The identity of the source and the temporal and spatial contexts of occurrence of the noises are present in subjects' cognitive representation and influence their perception. Experiments on alarm signals showed that contextual contraints influence the perceptual thresholds of the signals, their identification, and their efficiency (contrasting signals as such with nonmeaningful noises). In conclusion, such a "situated" cognitive (and pluridisciplinary) approach allows (i) at a theoretical level, to identify the roles of top down (high level) constraints on low-level perceptual processing and (ii) at a methodological level, it suggests to first design procedures to identify the meaningful categories of sounds and their properties at linguistic and psychological levels before describing them in physical dimensions and experimentally manipulating them in psychophysical paradigms.

\section{2:20}

4pNSc2. An intersensory approach to urban analysis and design. Marie-Christine Couic and Jean-Jacques Deletre (Ctr. de Recherche sur l'Espace Sonore et l'Environnement Urbain, Cresson, Ecole d'Architecture de Grenoble, BP 2636, 38036 Grenoble Cedex 2, France)

Cresson laboratory's research on sound in urban space led to the development of a theorical model called "sonic effect" that takes into account not only the physical and space aspects of the sound but also the user's perception. With this model, some interactions between senses were observed that have been analyzed from an intersensory standpoint. The first step of the method is to register on tape the feeling of candidates when walking through a selected area of the city. This raw material is then analyzed by dedicated software that extracts a few classes where expressions are closed from a semantic standpoint. These classes correspond to specific areas and overlap in transition zones where intersensory perception is expected. Physical measurements (sonic, thermodynamic, luminous) confirm these locations. Further analysis is then performed to reveal dynamic schemes of interaction. The evolution of the relationship between the most salient urban objects and the user is examined in order to consider the evolution between expression (description, evocation,....), motion, and senses. A limited number of protocols was found when meeting an object, but the articulation between the main components of intersensory perception (expression, motion, and senses) is still being analyzed. [Work supported by CNRS/PIR-VILLES.]

\section{2:40}

4pNSc3. Fractal modeling of diffusive urban configurations. Philippe Woloszyn (Cerma Lab., UMR CNRS 1663, Ecole d'Architecture, rue Massenet, F-44300 Nantes, France, woloszyn@cerma.archi.fr)

This contribution is based upon the environmental specificities of the urban built form and it is part of the Research Programme CNRS-Interdisciplinary Programmes for Towns "Towards an Urban Sonic Ambience Simulator." Its purpose is to develop an acoustic model based upon fractal measurements of urban interfaces, to be applied for sound simulations. The fractal technique 
exposed here for modeling the complexity of urban forms quantifies discrete morphologies of the town with an automatic capture procedure based on Minkowski's operators. In order to model the complexity of urban streets, the dilution measurement is given for a collection of urban surfaces with fractal indicators to evaluate the spectral diffuseness for every element. The implementation of the diffusive acoustic model will then be performed using finite-element software, composing the diffusive surfaces collection as a complex equivalent urban configuration. The final comparison with the results of on-site experimentations will give a spectral approach of the diffusion coefficient for the urban configuration. In the near future, the technique described in this paper aims at allowing the spectral control of the acoustical parameters by connecting a fractal morphological evaluation and a qualification of the acoustical phenomenon, modeled as a diffusive process.

\section{3:00}

4pNSc4. The University of Central Florida Rail Noise Model. Roger L. Wayson and John M. MacDonald (Univ. of Central Florida, Civil and Environ. Eng. Dept., P.O. Box 162450, Orlando, FL 32816-2450, wayson@ pegasus.cc.ucf.edu)

The Railway Noise Model (RNM) is a point source simulation model using a Windows-based program written in Visual Basic. Locomotives and rail cars are input by the laser using a graphical interface. The interface allows spreadsheet-type format or the user may use the mouse to drag and drop railroad tracks and barriers and to place receivers. The model has the ability to model heavy rail and light rail locomotives and rail cars. The user can observe defined trains moving along the railroad tracks to check input. $L_{\text {eq }}$ sound levels are calculated and reported during the simulation and then tabulated at the conclusion of simulation. The algorithms of the RNM follow the U.S. Federal Transit Authority Noise and Vibration Impact Assessment (DOT-T-95-16) guidelines. The Railway Noise Model uses $L_{\max }$ passby levels from REMEL curves to compute sound levels. The RNM simulates a 24-h period of rail traffic and computes $L_{\mathrm{dn}}, L_{\max }$, SEL, and $L_{\mathrm{eq}}$ levels at the receivers. Testing has shown that the RNM results match those of the FTA promulgated spreadsheet. The RNM is capable of modeling complex geometries and conditions not available in the FTA spreadsheet and supports all noise sources available in the spreadsheet.

3:20

4pNSc5. The University of Central Florida Community Noise Model. Roger L. Wayson and John M. MacDonald (Univ. of Central Florida, Civil and Environ. Eng. Dept., P.O. Box 162450, Orlando, FL 32816-2450, wayson@ pegasus.cc.ucf.edu)

The Community Noise Model (CNM) is a true simulation model written in Visual Basic allowing modeling of motor vehicles. User input is simplified by permitting spreadsheet input or graphical input using a mouse. Due to its simulation nature, the model handles interrupted flow traffic much better than conventional line source models. The model begins by using reference energy mean emission levels (REMELS) from a quite extensive measurement program. Propagation characteristics (geometric spreading, ground effects, atmospheric absorption, diffraction, etc.) are calculated at user-specified time step and energy is summed at user-defined locations. Using the acoustic energy summations, the model reports Leq, Lmax, and statistical levels. Traffic parameters are also reported. Validation has been performed as well as comparison to other established models and will be presented. The CNM performed quite well and at intersections outperformed other models when comparisons were done to measured data.

\section{Contributed Papers}

3:40

4pNSc6. Noise abatement in urban areas. A. A. F. M. Beeks, M. G. Dittrich, and P. P. Kooijman (TNO Inst. of Appl. Phys., P.O. Box 155, 2600 AD Delft, The Netherlands)

Two studies on noise abatement in urban areas, performed for the Dutch Ministerie of Transport (SSZ program), are presented. The first is a feasibility study on noise reduction of a range of vehicles. Buses and lorries were found to have comparable noise levels, whereas delivery vans and taxis were found to be much quieter. Specific noise sources can be high. More stringent (European) regulation or governmental stimulation measures are required. A survey was held amongst 396 inhabitants in two cities. Most noise disturbance was shown to be caused by buses (lowfrequency acceleration noise). The second study was on noise disturbance during goods delivery to shops. This was initiated after the extension of business hours and the introduction of stricter regulations $(60 \mathrm{~dB}$ peak limit). Current measured peak A-weighted noise levels at $7.5 \mathrm{~m}$ were found to vary between 60 and $95 \mathrm{~dB}$. The study concluded that noise control measures for most sources are technically achievable within three years. Initial estimates indicate $10 \%-20 \%$ higher investment on vehicles and handling equipment. Lorry diesel engine noise is about $15 \mathrm{~dB}$ too high. Well-designed quiet electric traction systems are a quieter alternative.
4:00-4:20 Break

\section{$4: 20$}

4pNSc7. Urban noise survey for the city of Porto Alegre, Brazil. Miguel A. Sattler (Univ. Federal do Rio Grande do Sul, Dept. de Engenharia Civil, Av. Osvaldo Aranha, 99-3o andar, Porto Alegre, RS, Brazil, CEP 90035-190)

This paper presents the methods and results of a simplified traffic noise survey for the city of Porto Alegre, the southmost capital city in Brazil. Noise measurement data and hourly vehicle flow data were used. Both were obtained at 6 p.m. Values from 560 sites were considered, making possible the construction of a simplified traffic noise map, built upon Leq values. The work, which tries to identify, for the stated time, the impact of noise on the population, in terms of the magnitude of the population exposed to different noise levels, has shown that $90 \%$ of the population included in the sample is exposed to A-weighted noise levels exceeding $65 \mathrm{~dB}$. 
on the method used (e.g., prefabricated or cast concrete). The emission data have been obtained from measurements at several construction sites,

4pNSc8. Planning and designing of developments against noise impacts-Hong Kong experience. Chee Kwan Lee and Kwok Keung Lau (Environ. Protection Dept., The Government of the HKSAR of the PROC, 46/F Revenue Tower, Hong Kong, cklee@epd.gov.hk)

In the next 15 years, there will be a rapid population growth in Hong Kong anticipated to be about 1.5 million people. It is a challenge for the Government of Hong Kong Special Administrative Region to find adequate land and to provide sufficient infrastructures to accommodate the increasing population while at the same time to keep up the environmental qualities. Owning to high demand for residential development, infrastructures, and other necessary facilities, it is not always possible to rely solely on buffer distance provided between noise-sensitive developments and noisy highways to avoid noise problems even for a piece of virgin land. To tackle the problems, due consideration needs to be given to minimize the potential noise impacts at the earliest planning stage when studying the alignments of highways. This paper presents the application of the above approaches in the planning of a 500 ha site.

\section{5:00}

4pNSc9. Local ordinance targeted to low-frequency noise. Bennett Brooks (Brooks Acoust. Corp., 27 Hartford Turnpike, Vernon, CT 06066, bbrooks@brooks-acoustics.com)

The local authorities of a suburban town wanted to control the lowfrequency noise emissions due to the musical entertainment at night clubs and taverns. Complaints from residents about booming bass guitar and drum sounds had to be balanced with the need for tavern owners to conduct business reasonably. It was recognized that the existing town code, which placed limits on A-weighted noise levels at property boundaries, was not an effective means to control low-frequency noise emissions. A revised ordinance was developed to address this issue, which now places additional limits on the allowable noise levels in specific octave bands. In particular, this includes the low-frequency (bass) octave bands. The octave band limits were selected such that the summation of band levels equals the A-weighted overall noise limit.

\section{$5: 20$}

4pNSc10. Collection data of noise emissions from building construction sites. Stefan Becker and Edelbert Schaffert (BeSB GmbH Berlin, Undinestr. 43, 12203 Berlin, Germany)

Until now, construction noise has mainly been looked at in terms of machinery. Several publications deal with the noise emission of construction machinery such as excavators, saws, etc. Government noise limits are normally given as average levels for daytime or night time. Thus (especially during the construction of the walls) noise predictions cannot be made on the basis of information of single tools only. Therefore a new study was initiated by the German Umweltbundesamt to collect data describing the total noise emission during the construction of buildings. The integrated approach has to be used, especially during the construction of the walls, because of the variety of simultaneous working processes. The study will determine the average sound-power level, information on sound impulses, and time information during each construction phase depending observation of the construction works, and discussions with constructionsite managers.

\section{$5: 40$}

4pNSc11. Models for acoustic sources in urban areas. David Gaulin and Michel Bérengier (Laboratoire Central des Ponts et Chaussées, Ctr. de Nantes, Rte. de Bouaye, BP 19, 44340 Bouguenais, France, Michel.Berengier@1cpc.fr)

Due to the large increase of populations in urban areas and the impact of sound phenomena on their life, the control of noise in cities is now of great importance. To predict acoustic fields for various urban situations, several theoretical approaches are currently under development. One of these is based on the diffusion theory [J. Picaut et al., Acust. Acta Acust. 83(4), 614-621 (1997)]. Whatever the model used, good predictions will be obtained only if the acoustic sources are accurately modeled. Those sources, classified following a qualitative approach, can be issued from traffic (cars, trucks, buses, motorcycles, etc.) but can also have human components (children in a school-yard, people in a market place, on a pedestrian street, ... ). The aim of this research concerns the modeling of each kind of source. The main parameters retained are the height, the directivity pattern, the sound power frequency spectrum, and the time history. The modeling techniques are based on statistical analysis and inverse fitting procedures on experimental data carried out over a large sample inside each source category. Afterwards, source characteristics will be introduced in the diffusion model and results will be compared with experiments performed on scale models and real urban situations.

\section{6:00}

4pNSc12. Sound field modeling in streets by a diffusion equation. Judicaël Picaut (Lab. Central des Ponts et Chaussées, Ctr. de Nantes, BP 19, 44340 Bouguenais, France, Judicael.Picaut@lcpc.fr) and Laurent Simon (Inst. d'Acoust. et de Mécanique, 72017 Le Mans Cedex 9, France)

By considering that surface irregularities of building facades are sufficient to produce diffusion in streets, the diffuse sound field modeling by the mathematical theory of diffusion is used to predict sound propagation and reverberation in rectangular streets. This modeling was first developed to predict the sound field in rooms with diffusely reflecting boundaries [J. Picaut, J.-D. Polack, and L. Simon, Acust. Acta Acust. 83(4), 614-621 (1997)]. In this paper, the modeling is applied to urban acoustics without significant modification. A 3-D diffusion equation is derived for the sound energy density and solved for time-varying sources and in steady state. A diffusion coefficient is introduced to characterize the degree of diffusion of the street, and an exchange coefficient depending on the absorption coefficient is used to take the wall absorption into account. When the diffusion coefficient is chosen correctly, predicted reverberation times and sound attenuation are in accordance with experimental data obtained in a scale model of a street. With new developments, by introducing in the model, for example, intersections, moving sources, meteorological effects...., this original modeling should be in the future, a simple solution to predict the noise impact in large urban areas. 


\title{
Session 4pNSd
}

\author{
Noise: Noise Emission From Machinery II \\ Hans G. Jonasson, Cochair \\ SP Swedish National Testing and Research Institute, Box 857, SE-50115 Boräs, Sweden \\ Angelo Campanella, Cochair \\ 3201 Ridgewood Drive, Columbus, Ohio 43026, USA
}

Chair's Introduction-1:55

Invited Papers

2:00

4pNSd1. A new hybrid approach, for estimating in situ the acoustic power radiated from vibrating structures. J. Nicolas, O. Beslin, and O. Foin (Mech. Eng. Dept., G.A.U.S. (Groupe d'Acoustique et Vibrations de l'Univ. de Sherbrooke), Univ. de Sherbrooke, Sherbrooke, QC J1K 2R1, Canada)

Measuring in situ, the acoustic power radiated by a vibrating panel with classical intensity techniques, is not always possible because of the influence of other surrounding sources. This is a major difficulty during the characterization in vehicles' noise problems. In this paper, a new experimental-numerical hybrid approach is presented which permits the evaluation in situ, of minimum and maximum indicators for the acoustic power intrinsically radiated by a particular panel situated in a more complex system. This is a two-step method: (i) the in situ vibration field of the panel is measured using a laser scan or an accelerometers array. (ii) Two indicators of the acoustic power are calculated. A minimum indicator is obtained by calculating the acoustic power radiated by the panel vibration field as if it were in free space (unbaffled vibration field). A maximum indicator is calculated considering a baffled vibration field (half free space). Interestingly enough, the measured data are projected in a base of trigonometric functions which allow quick calculations for a large frequency span. Then, precomputed baffled and unbaffled radiation impedances are used to calculate the radiated power. Comparisons between exact and estimated radiated powers are presented and discussed.

\section{2:20}

4pNSd2. Localization of noise sources inside machines by using a scaled dummy head. Helene Illaire and Jens Blomqvist (Dept. of Appl. Acoust., Chalmers Univ. of Technol., SE-41296 Gothenburg, Sweden, jens @ ta.chalmers.se)

This study presents the use of the fine spatial resolution of the human auditory system to localize sound sources in small enclosures, by means of a 1:10 scale dummy-head and subsequent real-time frequency scaling. This method has been applied to the localization of rattle sources in the gearbox of a truck. After investigation of cues necessary for localization in the horizontal plane in a reverberant environment, a dummy-head of a scale of 1:10 has been designed. The dummy-head reproduces the general shape of the human head but the pinnae are not found necessary for inclusion for this investigation at low frequencies. To preserve the ability to localize, the frequency content of the recorded signal is scaled by the same factor as the dummy-head, i.e., it is divided by a factor of 10. Therefore, a frequency divider system has been developed to divide the frequency in real-time by 10 and uses a time-expansion method. The design of the system and the problems encountered are presented, together with the results obtained.

4pNSd3. Order tracking of diesel engines with a Kalman filter. Krystof Kryniski and Ulf Carlsson (MWL, KTH, Stockholm S 100 44, Sweden, kk@FKT.KTH.SE)

Order tracking analysis is one of the techniques used to monitor the performance of diesel engines. The objectives are to separate vibration into terms corresponding to the rotational speed and its multiples and then to monitor the changes over the revolution of a crankshaft. A Kalman filter provides the technique of isolating each of the components that are synchronous to the shaft rotation. By filtering the signal in the time domain it has also the capabilities to follow any variation of the rotational speed. Thus, the Kalman filter, if properly designed, can provide a tool for testing not only steady engine operation but can also be used to analyze both the engine acceleration and declarations under different loads. The paper discusses first, the process of monitor the frequency components locked up to the rotational speed with high resolution. Then, it shows how to reconstruct the synchronous components, in terms of amplitude and phase, to produce the vibration patter over the crankshaft revolution. 
4pNSd4. Characterization of I. C. engines as a linear acoustic source using internal measurements. Slaheddine Frikha, Samir Boukhari (Laboratoire de Mecanique Physique, UPMC, CNRS Upresa 7068, France, frikha@ccr.jussieu.fr), Xavier Mouton (Renault, Guyancourt, France), and Arnauld Vitel (PSA, La Garenne, France)

Nowadays, faced with international competition, the vehicle constructors are constrained to reduce their studies' periods and costs. The knowledge of engine acoustic properties as a source exciting its inlet and exhaust pipes is required to perform predictive computations. Present here is a new method allowing engine source characterization as an acoustic pressure source having finite impedance that consists of fitting the engine nonlinear behavior by an equivalent linear one. It is based on a multi-charges approach. Internal pressures at a few points of a set of silencers are used to identify the pressure and the velocity at the outlet of the catalytic converter. The main future of the method is its ability to characterize the engine at realistic functioning conditions. In particular, the inverse model does not include a tail pipe radiation model, so the back pressure may be imposed to a realistic one. That reduces the effects of nonlinearity of the engine and increases the accuracy of the identified source. The internal pressure data are obtained by real measurement since a prototype of the engine exists. Otherwise, they can be computed which allows beginning the design of the exhaust system at an early stage of engine designing.

4pNSd5. Experimental study of rasping noise in internal combustion engine exhaust systems. Mondher Ayadi, Slaheddine Frikha, Pierre Yves Hennion (Laboratoire de Mecanique Physique, UPMC, CNRS URESA, 7068 Paris, France, ayadi@ccr.jussieu.fr), and Jean Michel Coulon (Arvin Exhaust System, Preston, UK)

The abnormal noise, known as rasping noise by several exhaust muffler designers, is a metallic intermittent noise which irritates the ear and has an adverse effect on vehicle marketability. It occurs in small cars having no more than two boxes in their exhaust systems. This phenomenon is highly noticed during a rapid acceleration or deceleration in cold conditions. However, it is sharply attenuated when the exhaust system is warmed up. An experimental study has been undertaken. Several exhaust systems, ranging from a simple pipe to a complete exhaust line, are tested. Pressure measurements in the line are performed by piezoelectric transducers. The tail pipe noise measurements are performed by a microphone. It has been observed that, unlike the usual exhaust noise that contains essentially second and fourth orders, the perception of the so-called rasping noise is associated with high-frequency components in the signal and needs higher-order analysis to be fully characterized. The experiments also show the steepening of the waves when rasping noise is listened. The high axial temperature gradient causes an increasing of that effect and probably a generation of shock waves which excite the main muffler and cause the metallic noise.

4pNSd6. Power output of a machine operating near elastic structures. Tatiana M. Tomilina, Michael P. Korotkov, and Vladimir B. Yashkin (Lab. of Structural Acoust., Mech. Eng. Res. Inst., M. Kharitonievsky 4, Moscow 101830, Russian Federation, tatiana@cgrsmx.iki.rssi.ru)

For predicting the noise levels produced by machinery, techniques, like SEA, based on the energy balance equations in which the radiation power of each noise source (machine) is used as an input parameter, are often employed. Usually, the standard value of the power measured in the free space is taken and it is assumed independent of acoustic environment. In the present work, it is shown experimentally and in computer simulation examples that the power output of an extended noise source operating near an elastic structure can differ significantly (several orders of magnitude) from its standard value. Possible corrections for the energy techniques are discussed.

\section{4:00}

4pNSd7. Development of a high-frequency precision anechoic test facility. John Duda, Martin Hirschorn (Industrial Acoustics Co., 1160 Commerce Ave., Bronx, NY 10462), Mark Gilbert, and Guy Torio (Shur Brothers, Evanston, IL 60602)

Although anechoic wedges with absorption coefficients of 0.99 as tested in typical impedance tubes meet ISO inverse square-law standards, they may not be satisfactory for anechoic rooms used for measurements of microphone response characteristics. Due to size restrictions, impedance tube tests are limited to frequencies below about $250 \mathrm{~Hz}$ and there is no standard method to test wedges for absorption at higher frequencies. This paper describes how pulse signal and reverberation room tests were employed to rank the high-frequency performance of three types of anechoic wedges considered for a microphone test facility: Standard patented Metadyne TM (perforated metal) wedges - conventional fiberglass wedges and an improved high performance Metadyne wedge system. The results of laboratory tests and tests conducted in anechoic rooms are presented. In all cases the preferred system, high-performance Metadyne wedges, is shown to meet the stringent acoustic absorption requirements needed for this application. The paper indicates the need for new standards for laboratory testing of anechoic wedges at high frequencies and an alternate method to inverse square-law tests for evaluating anechoic room performance such as stepped frequency sweep tests. Such a new standard will be proposed in the presentation. 


\section{Contributed Posters}

These posters will be on display in the Poster Gallery from Thursday to Friday, 18-19 March. Authors will be at their posters from 4:40 p.m. to 6:20 p.m. on Thursday, 18 March.

4pNSd8. Characteristics of MRI acoustic noise in functional and high-resolution imaging of the auditory pathways. Uwe Baumann (Dept. of Audiol., Ludwig-Maximilians-Universität, Marchionini-Str. 15, 81377 München, Germany) and Lorenz Jäger (Ludwig-Maximilians-Universität, 81377 München, Germany)

The acoustic noise generated by magnetic resonance image (MRI) systems causes problems for the measurement of funtional responses to acoustic stimuli. The time structure and spectral composition of the MRI noise depends mainly on the sequence of gradient switching. As a rule of thumb, faster switching produces more intense noise than slower switching. Functional and high-resolution MRI of the auditory pathways require fast switching sequences to ensure high place and time resolution. To investigate the characteristics of the MRI noise, five different sequences (EPI-fMRI, 3D-MPRAGE, 3D-CISS, 2D-FLASH, EPI-DIFF) were recorded on a 1.5-T device (Siemens Vision) at the position of subject head and also $1.2 \mathrm{~m}$ outside the MRI opening to estimate artifacts caused by direct induction into the microphone (B \& K 4165). The measured peak SPL inside the MRI varied between 101 and $130 \mathrm{~dB}$ depending on the sequence, but a comparison with the results outside the MRI cabinet (87$100 \mathrm{~dB}$ SPL peak) revealed for the 3D-CISS and EPI-DIFF sequence an influence of direct induction with an amount up to $20 \mathrm{~dB}$. An ear-related spectral analysis and a virtual pitch calculation was performed to estimate the amount of tonality of each sequence.

4pNSd9. Adaptive decision method for acoustical turbomachinery diagnostics. Olena N. Bezvesilna (Dept. of Devices, Natl. Tech. Univ. of Ukraine, 37, Peremogy pr., Kiev, 252056, Ukraine) and Leonid M. Gelman (National Tech. Univ. of Ukraine, Kiev, 252056, Ukraine)

Acoustical turbomachinery diagnostics is advisable to do automatically and complexly using simultaneously different methods. The mentioned diagnostics is condition monitoring diagnostics. Such diagnostics is necessary to do at all operation regimes of turbomachinery and therefore with changes of interfering data of turbomachinery and of environment condition in which diagnostics is carried out. However, in the presence of variable interfering data, optimum properties of decision methods are not realized. In addition, features used for diagnostics are usually statistically dependent. To provide diagnostics in the mentioned conditions it is necessary to apply the adaptive approach. A new automatic adaptive sequential method for acoustical turbomachinery diagnostics is proposed and developed for: (a) presence of variable interfering data; (b) changes in the space of these data limits of erroneous test probabilities; (c) availability of statistical dependencies between features. A new procedure to account for the statistical dependencies between features is proposed and developed. Acoustical diagnostics of the gas-turbine engine based on noise emission is presented. [Work supported by the MacArthur Foundation.]

4pNSd10. Investigations of sound insulation of partitions used for integrated enclosures. Zbigniew Engel and Jan Sikora (Dept. of Mech. and Vibroacoustics, Univ. of Mining and Metallurgy, al. Mickiewicza 30, 30-059 Krakow, Poland, engel@uci.agh.edu.pl)

Integrated enclosures, rarely applied in practice, are useful in the reduction of the excessive noisiness of machines and equipment. They are especially important for the machinery requiring constant and direct operation and maintenance, for which the application of other enclosures (e.g., fully closed) is inadmissible. Results of the sound insulation investigations of new types of partitions designed for the construction of integrated enclosure elements have been presented in the paper. Several specimens of partitions, which, according to their acoustic properties belong either to single (homogeneous and heterogeneous) or to multiple partitions (consisting of single, mixed and layered ones), were prepared for experiments. Double partitions with air cavities either empty or filled with sound absorptive materials, rigid, elastic, or granular absorbers (damping the airborne and material sound levels), appeared to be the main solution. The best partitions were used in the prototype of the integrated enclosure constructed for the engine lathe testing. The mechanical noise level of the lathe was reduced by $15 \mathrm{~dB}$. The sound-insulating performance of the prototype has shown that the construction of integrated enclosures can be utilized in designing the new machines characterized by the lower acoustic activity - in comparison with the present standards. 


\title{
Session 4pPAa
}

\section{Physical Acoustics and Engineering Acoustics: Numerical Methods for Computing Sound Radiation and Scattering II}

\author{
Martin Ochmann, Cochair \\ Technische Fachhochschule Berlin, Fachbereich Mathematik und Physik/Fernstudien Institut, Luxemburgerstrasse 10, \\ D-13353 Berlin, Germany \\ Gary H. Koopmann, Cochair \\ Department of Mechanical Engineering, Pennsylvania State University, 157 Hammond Building, University Park, \\ Pennsylvania 16802, USA
}

Invited Papers

2:00

4pPAa1. Application of wavelet decomposition (space-wave number) to sound radiation 1D. M. Martinet, N. Hamzaoui, and J. L. Guyader (Laboratoire Vib. Acoustique de L'INSA de Lyon, 20 avenue A. Einstein, 69621 Villeurbanne Cedex, France)

The purpose of this presentation is to study the contribution of a multiresolution analysis based on a decomposition in wavelets, so as to predict the acoustic radiation of a vibrating structure. In order to analyze the sensitiveness of different parameters of this approach, it is suggested that it be applied to the radiation of a heterogeneous beam in flexion. After an analysis of the radiation associated with different forms of wavelets, the displacement field of the beam is decomposed on a wavelet basis, and its radiation will be then computed and faced with a result stemming from a classical technique. First, the interest, researched by this approach, is linked to the reduction of the number of points of mesh of the vibratory field of the beam, and second, to a physics analysis giving way to access to the spatial localization of the radiated parts. The formulation of the one-dimensional problem, the physics concepts of the multiresolution analysis, and a confrontation with a bibliographic reference, analyzing the advantages and the drawbacks of this approach, are thus presented here.

2:20

4pPAa2. BEM and the use of explicit frequency-dependent matrices-A study on computing wave scattering and radiation. Steffen Marburg (Institut für Festkörpermechanik, Technische Univ., D-01062 Dresden, Germany, marburg@mfm.mw.tu-dresden.de)

The use of explicit frequency-dependent matrices as in finite-element methods has not really been established in boundary element methods yet. The particular integral method (PIM) that may be applied to construct explicit frequency-dependent matrices has already been tested for the solution of interior problems in structural acoustics. However, a formulation for the exterior problem using PIM has not been published. In this paper, the author will discuss ways to apply the PIM to compute wave scattering and sound radiation.

2:40

4pPAa3. A surface element formulation of the equivalent source method. U. Peter Svensson (Dept. of Appl. Acoust., Chalmers Univ. of Tech., SE-41296 Gothenburg, Sweden), Mayumi Nakano, Kimihiro Sakagami, and Masayuki Morimoto (Kobe Univ., Kobe 657-8501, Japan)

A time-domain formulation of the equivalent source method which uses planar surface elements is presented. The boundary condition of a closed surface can be fulfilled using monopole elements for boundary conditions specifying the normal velocity, the sound pressure, or the local impedance. By interpreting the equivalent sources as double layers of free-field radiating monopoles with a uniform source signal over the element, the impulse response matrix is easy to find via the Rayleigh integral. The problem of determining the source signals of these equivalent sources involves the inversion of a single real-valued matrix as shown in Kropp and Svensson [Acustica 81, 528-543 (1995)], and the matrix is very sparse. The relation to the boundary element method is discussed, and as an example, the sound radiation from a kettledrum is presented. By using a Green's function technique, any vibration pattern can be synthesized using post-processing. Interpolation can be used to study the far-field radiation of vibration patterns that are more complicated than the element mesh can resolve.

3:00

4pPAa4. Two surface element formulations in the time domain for the calculation of sound fields in cavities with varying boundary conditions. Wolfgang Kropp and U. Peter Svensson (Dept. of Appl. Acoust., Chalmers Univ. of Technol., SE-41296 Gothenburg, Sweden)

Two different surface element formulations are presented which apply the equivalent source method in the time domain. The first method uses planar surface elements. The boundary condition of the cavity surface in the form of a prescribed impedance is fulfilled by using monopole elements (i.e., layers of free-field radiating monopoles with a uniform signal over the element). The second 
method is based on the Kirchhoff-Helmholtz integral. While the traditional boundary element technique uses the free-field Green's functions, here a set of specialized Green's functions is applied which is adapted to the cavity geometry. This set of functions fulfills the boundary conditions for the cavity with rigid walls. In this way the Kirchhoff-Helmholtz integral is simplified substantially and only areas of the surface with nonrigid boundary conditions have to be included in the calculations. Advantages and disadvantages of both formulations are highlighted, and results from both methods are compared for rooms of different complexity.

3:20

4pPAa5. Using the equivalent sources method (SUP) for multibody radiation problem. Tatiana M. Tomilina (Lab. of Structural Acoust., Mech. Eng. Res. Inst., M. Kharitonievsky 4, Moscow 101830, Russian Federation, tatiana@cgrsmx.iki.rssi.ru)

The radiation problem for a finite body vibrating in a fluid near rigid scatterers and for elastic structures is discussed. It is shown that the ESM is the most appropriate method for treating the problem. Formulation of the boundary conditions on the surfaces of elastic scatterers is the key point of the method here. New results of computer simulation and laboratory experiment concerning the energetics of sound radiation are presented. The comparison of two modifications of the ESM is considered in this paper: one modification is based on the Rayleigh multipole expansion and the other uses the equivalent sources of the monopole type.

3:40

4pPAa6. Efficient boundary element calculations using thin-plate acoustic elements. John B. Fahnline (Appl. Res. Lab., 16 Appl. Sci. Bldg., University Park, PA 16802, jbf@ wt.arl.psu.edu) and Gary H. Koopmann (Ctr. for Acoust. and Vib., University Park, PA 16802)

Thin-plate elements are commonly used to represent structural components whose thicknesses are much smaller than the structural wavelength. This simplifies the analysis, because otherwise several elements are required to represent correctly the strain distribution through the thickness, leading to much larger models. Thin-plate elements also have advantages when they are used in acoustic boundary element calculations. By using dipole sources at the midline of the elements to generate the basis functions for the acoustic analysis, the boundary condition can be enforced such that the opposite sides of the plate are forced to move together. The surface velocity boundary condition then only has to be enforced over one side of the elements (even though both sides are in contact with the acoustic medium). Mechanical radiation impedance calculations can also be simplified because the dipole source basis functions create the same pressure field on opposite sides of the plate elements and thus impart no force to the structure. The only exception is for "self-element" terms, where the dipole sources create equal, but opposite, surface pressures. Several example problems are used to validate the calculations, including a vibrating strip, a transversely oscillating disk, and a cantilever plate.

\section{Contributed Papers}

4:00

4pPAa7. Finite-element methods applied to the propagation of acoustic wave along immersed wedges. A. C. Hladky-Hennion, P. Langlet, R. Bossut (IEMN, département ISEN, 41 boulevard Vauban, 59046 Lille Cedex, France), and M. de Billy (Université Paris 7, 75251 Paris Cedex 05, France)

Immersed in water, wedges can generate either propagating or radiating acoustic guided waves according to the value of the apex angle. The propagation of such waves is analyzed with the help of two methods based on finite-element methods: a time analysis [A. C. Hladky-Hennion et al., J. Acoust. Soc. Am. 104, 64-71 (1998)] and a modal analysis for which an original numerical technique is suggested to describe the radiating (or supersonic) modes. The finite-element results obtained with the two methods for brass and duraluminum wedges are compared to the experimental measurements. A good agreement is observed for either subsonic or supersonic regions.

\section{4:20-4:40 Break}

\section{4:40}

4pPAa8. A combined finite-element, boundary integral, and spherical harmonic hybrid technique for computing multiple-body sound radiation. John B. BlottmanIII and Anthony J. Kalinowski (Naval Undersea Warfare Ctr. Div. Newport, 1176 Howell St., Newport, RI 02841-1708, blottman-john-b@ code21.npt.nuwc.navy.mil)

Transducer interactions within an array are known to affect the array performance and the life expectancy of the transducers. The ability to predict array performance is required for large close-packed arrays of acoustic transducers. In these cases, traditional full finite-element methods prove costly. A combined multiple acoustic scattering model that is based on spherical harmonic functions and a finite-element-based description of an elastic body of arbitrary geometry is formulated to predict the performance of an array of acoustic transducers. The finite-element model produces the dynamic system matrix. A boundary integral equation relates surface pressure and velocity. Boundary elements are partitioned for the case of the surface of an arbitrary body circumscribed by a spherical surface to provide an implicit transfer of surface pressures to spherical fluid pressures and particle velocities. These quantities are then transformed in terms of spherical harmonics. The resulting series is combined with the spherical harmonic representation of the fluid using the addition theorem for multiple scatters. The technique has been exercised to evaluate an array of piezoelastic shells, driven by an electrical potential. Results compare well to those of a coupled finite-element-boundary-element method. [Work sponsored by the Office of Naval Research.]

\section{5:00}

4pPAa9. A reflection free boundary condition for propagation in uniform flow using mapped infinite wave envelope elements. Walter Eversman (Mech. and Aerosp. Eng. and Eng. Mech., Univ. of MissouriRolla, Rolla, MO 65401, eversman@umr.edu)

A new reflection free boundary condition based on mapped infinite wave envelope elements is developed for finite-element modeling of acoustic radiation in a uniformly moving medium. These elements are useful for obtaining closure for computations on an infinite domain in which a radiating body is immersed in a moving medium which is essentially undisturbed outside of the near field. The application described is for harmonic excitation which leads to a model independent of time. An additional result of this study shows that the mapped wave envelope elements provide a boundary condition equivalent to stiffness, mass, and damping matrices appended to the inner mesh. By choosing the transition between the standard FEM mesh and the mapped WE as a surface of constant phase, the mass matrix is caused to vanish identically. This has implications for transient FEM modeling of acoustic radiation. A demonstration of the characteristics of the mapped WE elements is given in the 
context of acoustic radiation from a turbofan inlet for which benchmark results are known. It is found that for similar computational cost mapped infinite wave envelope elements are superior to conventional wave envelope elements for time-independent formulations.

\section{$5: 20$}

4pPAa10. How reliable is the method of comparative sources? KaiUlrich Machens $^{\text {a) }}$ (Inst. of Tech. Acoust., Tech. Univ. of Berlin, Berlin, Germany, kamachen@gfn.daimler-benz.com)

Radiation and scattering from vibrating bodies can be expressed by a source system that is placed inside the radiator and whose source strengths are determined appropriately. The method of comparative sources (MCS) provides an estimate of these source strengths from the near-field data. The MCS is reviewed by applying it to cylinders with elliptical cross section. This geometry is suitable to check the method for two reasons. First, it is general enough to demonstrate methodical difficulties which arise from its spatially varying field impedances as a result of different surface curvatures. On the other hand, it is not too complicated to allow analytical treatment by Mathieu functions. Therefore, it is possible to generate exact solutions of the sound field from different, more and less efficiently radiating velocity distributions. These solutions are compared to results obtained by the MCS, in which various source systems and different weighting functions are used. This way, it is demonstrated that the relative error in the surface velocity approximation generally is not proportional to the error in the radiated sound power. Moreover, it is shown that certain weighting functions may be advantageous and that it is more difficult to predict inefficient than efficient radiators by this method.

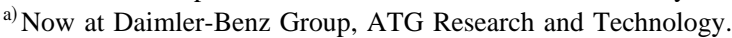

\section{$5: 40$}

4pPAa11. Designing electrodynamic loudspeakers by using a new computer modeling scheme. Martin Rausch, Reinhard Lerch, Manfred Kaltenbacher, Hermann Landes (Dept. of Elec. Measurement Technol., Univ. of Linz, Altenbergerstr. 69, A-4040 Linz, Austria, Martin.Rausch@jk.uni-linz.ac.at), Gerhard Krump, and Leonhard Kreitmeier (Harman Audio Electron. Systems GmbH, D-94315 Straubing, Germany)

A new technique for the computer modeling of fluid-loaded magnetomechanical transducers is presented. This numerical technique is based on a finite-element method (FEM) and allows the precise calculation of the magnetic, mechanical, and acoustic fields, including their couplings. Fur- ther on, nonlinear effects in the mechanical and magnetic behavior are considered. Therewith, the complex dynamic behavior of electrodynamic loudspeakers was studied and, furthermore, an appropriate computer-aided engineering (CAE) environment has been established. This CAE tool allows an optimization of critical parameters, for example: The validity of the computer simulations is verified with the help of appropriate measurements. The calculated and measured values for eigenfrequencies, axial pressure responses, and electrical input impedances are in excellent agreement. The applicability of the presented calculation scheme with respect to the computer-aided design of moving coil drivers is demonstrated by reporting several practical applications, which are of great interest for loudspeaker manufacturers. In the present paper, the elimination of response dips at intermediate frequencies, the calculation of polar responses, and the examination of the so-called jump-out excursion, are presented.

\section{6:00}

4pPAa12. Acoustic photo- and cinematography based on interference transformation. Gerd K. Heinz, Thanh T. Nguyen, and Dirk Duebler (Gesellschaft zur Foerderung angewandter Informatik e.V., D-12484 Berlin, Rudower Chaussee 5, Germany)

A time-domain technique is introduced to localize far and moving acoustic objects (compare URL http://www.gfai.de/www_open/perspg/ heinz.htm). The algorithm is called "Heinz Interference Transformation" (HIT) [Heinz et al.: "Time pattern, data addressing, coding and topographic maps between multiple connected neural fields-a physical approach to neural superimposition and interference," BioNet'96, Berlin, ISBN 3-00-001107-2]. HIT reproduces mirrored (optical) projections just as nonmirrored reconstructions of different sources depend on the direction of time axis. Based on time-domain calculations, the introduced method reproduces an approximation for different source points in a grid matrix, arranged in an virtual space or array or field in physical dimensions of the measured scene. It is possible to reconstruct acoustic singletime events to cinematographies (movies). Maximum image rate is the sample frequency of time functions. Independent of the integration inter$\mathrm{val}$, it is possible to reconstruct in cinematographies the wave field or movies. Properties of HIT, influence of time functions' properties and channel number, different algorithmical tasks, relations between time and space, aliasing and reconstruction parameters are discussed as examples. [Work supported by German BMWi, Marktvorbereitende Industrieforschung.]

\section{Invited Poster}

This poster will be on display in the Poster Gallery from Thursday to Friday, 18-19 March. Authors will be at their posters from 10:00 a.m. to 12:00 noon on Friday, 19 March.

4pPAa13. Some aspects of the scattering calculation using the source simulation technique. Paulo Henrique T. Zannin (Dept. Engenharia Mecanica, Univ. Fed. Parana, Centro Politecnico, Curitiba, PR 81531-990, Brazil)

In this work the source simulation technique was used to calculate the scattering of a plane wave by a cylinder with radial or elliptical transversal section. Numerical simulations have shown that: (1) the shape of the auxiliary surface, (2) the number of sources, and (3) the way the sources are distributed are the most relevant parameters to ensure an accurate solution for the problem. In the case of the single-layer method, sources should not be positioned close to the surface or to the center of the body, because the problem becomes ill-conditioned. The auxiliary surface and the scatterer should be as similar as possible in order to minimize the boundary error. With respect to the number of sources $(N)$, there are two opposite effects: (1) if $(N)$ is too small, the sound field is not reproduced accurately, (2) if $(N)$ is too large, computing time increases and solution accuracy decreases. The method breaks down when excitation frequency coincides with the eigenfrequencies - a narrow range of frequencies — of the space formed by the auxiliary surface. As the auxiliary surface is frequently represented by simple surfaces (cylinder, sphere), one can easily calculate the eigenfrequencies, and therefore avoid them. 


\title{
Contributed Poster
}

This posters will be on display in the Poster Gallery from Thursday to Friday, 18-19 March. Authors will be at their posters from 10:00 a.m. to 12:00 noon on Friday, 19 March.

4pPAa14. Numerical modeling of axisymmetric sources in infinite baffle. Bronislaw Zoltogorski, Andrzej Dobrucki, and Cecylia Szmal (Wroclaw Univ. of Technol., Inst. of Telecommunications and Acoust., Wybrzeze Wyspianskiego 27, 50-370 Wroclaw, Poland, brz@zakus.ita.pwr.wroc.pl)

According to standards, characteristics of loudspeakers should be measured in an anechoic room with the use of sufficiently great rigid baffle. To achieve accordance of numerical calculations to results of measurements, the numerical algorithms for a source in an infinite baffle are needed. In the paper three boundary integral methods - the Huygens-Rayleigh (HR) integral method, the Kirchhoff-Helmholtz $(\mathrm{KH})$ integral method, and the simple-source density (SSD) method-are explored for the sources of revolution with optional surface velocity distributions and shapes of cross sections (cone, flat piston, dome). It was shown that solution of the boundary-value problem for the KH and SSD methods exists and is unique for all frequencies. The HR method is numerically the simplest and is free of existence/uniqueness problems but is theoretically founded for a flat piston only, whereas for sources of other shapes it gives erroneous results. The problem of inclusion of an infinite baffle into the numerical model is easy to overcoming for the SSD method because an auxiliary sourcedensity function rapidly decreases out of source and only a small fragment of the baffle influences the final result. For the KH method that question is more difficult because pressure on the baffle surface does not decrease sufficiently fast. The improved KH method that overcomes this deficiency is described.

THURSDAY AFTERNOON, 18 MARCH 1999

ROOM MA041, 2:00 TO 6:20 P.M.

\section{Session 4pPAb}

\section{Physical Acoustics: Measurement and Evaluation of Ultrasound II}

\author{
Rainer Reibold, Cochair \\ Physikalish-Technische Bundesanstalt, Bundesalle 100, D-38116 Braunschweig, Germany \\ R. C. Chivers, Cochair \\ 6 Louis Fields, Guildford GU3 3JG, UK
}

Contributed Papers

\section{2:00}

4pPAb1. Calibration of contact transducers by means of thermoacoustic sensors. Michael Glinka (Physikalisch-Technische Bundesanstalt, Labor 1.43, Bundesallee 100, 38116, Braunschweig, Germany)

The utilization of the thermoacoustic effect for measuring the absolute value of acoustic power by means of thermoacoustic sensors is discussed. The sensor's response to the incident acoustic power is measured as the temperature increase $T$. This temperature increase is computed by the differential equation describing the sensor, which contains only the sensor's material constants: density $\rho$, specific heat capacity $c_{w}$, and conductivity $\Lambda_{w}$,

$$
\frac{\partial T}{\partial t}=\frac{\Lambda_{w}}{\rho c_{w}} \nabla^{2} T+\frac{1}{\rho c_{w}} \cdot W .
$$

It is shown how the distribution of heat sources $W$ (caused by the absorbed ultrasonic field) can be optimized for the realization of thermoacoustic sensors adapted to experimental situations. A discussion of different types of sensors will show that the complex transfer functions are restricted by the material constants. It is shown how the solutions of (1) presented can be used to improve the dynamic performance. Experimental results obtained by the use of different sensors and contact transducers (calibrated by the reciprocity method) are compared with respective solutions of the parabolic heat flow equation (1).

\section{2:20}

4pPAb2. Improvement of the lateral resolution of finite-size hydrophones. Tarek Boutkedjirt and Rainer Reibold (Physikalisch-Technische Bundesanstalt, D-38116 Braunschweig, Germany)

In recent years, there has been an increasing number of ultrasound applications which utilize frequencies well above $10 \mathrm{MHz}$. Piezoelectric hydrophones are commonly used for the characterization of the respective fields. With the exception of specially designed laboratory devices, limits are set to the hydrophones currently available due to their finite size. Instead of measuring the acoustic pressure at a point, these devices furnish a value integrated over the sensitive aperture. The adverse averaging effect is particularly prominent in the Fresnel zone of the sound field and at frequencies above $10 \mathrm{MHz}$, where the aperture dimensions are of the order of several acoustic wavelengths in water. This problem can be solved by numerically deconvolving the aperture function from the observed response of the finite-size receiver. The efficiency of the deconvolution process is shown for both numerical simulations and experimental investigations. Special regard is paid to the methods of noise reduction by means of averaging, smoothing, and filtering. 
input admittances of the transducers are theoretically regenerated by the

4pPAb3. The use of the finite-element method to analyze the influence of depolarization by structuring to the performance of $2 \mathrm{D}$ arrays. Daniel Schmitt, Peter K. Weber (Dept. of Ultrasound, FH-IBMT, Ensheimerstr. 48, D-66386 St. Ingbert, Germany, peterw@ibmt.fhg.de), Roger W. Whatmore, and Chris Goat (Cranfield Univ., Cranfield, UK)

3-D ultrasonic imaging can be achieved by steering conventional single-element scanners (pseudo 3-D imaging). The use of 2-D arrays offers a wide range of possibilities for electronic focusing and steering, which will lead beyond the limitations of pseudo 3-D imaging. Measurements of single-array elements have shown that the method of structuring, like dicing, and its process parameters, leads to a decreased coupling factor. This will result in a loss of efficiency and bandwidth. Because this effect can be reduced by repolarizing the array, one can assume a depolarization of a certain depth along the cutting edge of the material. It is very difficult to determine the degree of depolarization and the depth of this dead zone. Using simple geometric models with good aspect ratio and a completely depolarized death zone, the influence of its depth on the coupling factor can be determined. These presumptions are not valid for most 2-D arrays in ultrasonic imaging. Their complex geometry and parameter combinations can be analyzed by the finite-element method. Results obtained from finite-element simulations that are in good agreement with the measurements will be presented and compared with other methods.

\section{3:00}

4pPAb4. Investigation of effective geometrical parameters for a pulseecho linear array with cylindrically curved, focusing surface. Ping Wu and Tadeusz Stepinski (Uppsala Univ., Dept. of Mater. Sci. Signals and Systems, Box 528, S-751 20 Uppsala, Sweden)

Linear arrays with cylindrically curved, focusing surfaces working in pulse-echo mode are currently being used for applications in nondestructive testing. Such commercial arrays are usually specified with nominal geometrical parameters (GPs), such as width, length, and radius of curvature (focal length), which, when used in the conventional piston source model, may result in an inaccurate prediction of the actual radiated field. Like a spherical focused transducer which has been extensively investigated for determining its effective GPs, a linear array with cylindrically curved, focusing surface has its effective GPs which allow the piston source model to provide a more realistic prediction of the actual radiated field. The goal of this paper is to investigate effective GPs for such a linear array used in pulse-echo mode. The effective GPs are determined by comparing the fields predicted by the extended angular spectrum approach (ASA), which is applicable to curved transducers, with the measured fields. Pulse-echo fields from the array were measured in water using a pointlike scatterer, and calculated using both nominal and effective GPs in the extended ASA. The results show that effective GPs do permit the piston source model to yield a more precise prediction of the measured fields.

\section{3:20}

4pPAb5. Characterization of an ultrasound system. Steinar Vervik and Magne Vestrheim (Dept. of Phys., Univ. of Bergen, N-5007 Bergen, Norway)

Frequency responses of an ultrasound system consisting of sender electronics, sender transducer, air as coupling medium, receiver transducer, and receiver electronics are measured and theoretically regenerated in the range 170 to $270 \mathrm{kHz}$. Magnitude and phase of the sender and receiver sensitivities are estimated by utilizing the reciprocity principle in combination with models and measurements of the transducer-transducer coupling function and the frequency response and loading effects of the electronics. Voltage-to-particle velocity frequency responses and electrical use of experimental modal analysis methods. The sound speed and absorption coefficient are estimated with diffraction corrections through spectral analysis methods. Simulated and measured waveforms are compared. Using time-frequency analysis, mode effects are studied and the waveforms and delays are related to the different functional blocks of the system. Differences between the measured and simulated transit times are found to be of the order of $10 \mathrm{~ns}$ in the range considered. The results are of interest in the analyses of zero-flow performance of ultrasound flow meters, in, for example, the study of signal detection criteria and in analyses of delays caused by nonfluid parts of the measurement system.

\section{3:40}

4pPAb6. A unified linear system approach to ultrasonic radiation from a baffled transducer. Kathy J. Hays-Stang (Univ. of Texas at Arlington, Arlington, TX)

An acoustic disturbance can be viewed as a waveform in space and time, or by the temporal or spatial frequency content of the acoustic disturbance. For each waveform and frequency viewpoint, the individual Green's function for acoustic radiation from a baffled transducer is available in the literature. The unified approach allows the Green's function for any given viewpoint to be transformed appropriately to obtain the Green's function for a desired viewpoint (personal conversations with the late Bill D. Cook). Given a Green's function for the acoustic radiation in a linear acoustic system, and the impulse responses of the other system components, the output can be determined for a given input. Choice of the most advantageous radiation impulse response/transfer function can reduce calculation time or provide physical insight for a problem of interest.

\section{4:00}

4pPAb7. Application of a fiber-optical probe hydrophone for in vivo measurements of therapeutical ultrasound. Thomas Dreyer, Rainer E. Riedlinger (Inst. fuer Hoechstfrequenztechnik u. Elektronik/Akustik, Univ. Karlsruhe, D-76128 Karlsruhe, Germany, Thomas.Dreyer@etec.uni-karlsruhe.de), Johannes Zenk, and Heinrich Iro (Univ. des Saarlandes, D-66421 Homburg, Germany)

Knowledge of sound fields used in therapeutical applications with focused strong ultrasonic pulses in vivo is of great importance to the user and researcher. In this study the ultrasonic field of an experimental high energy pulsed ultrasound (HEPUS) device for tumor treatment is investigated. Using HEPUS, the mechanism of acute tissue destruction is based mainly on cavitation effects in the focal region. A self-focusing piezoelectric transducer generates the applied pressure signal which is a highamplitude sinusoidal burst with a duration of about $12 \mu \mathrm{s}$. These burst signals differ significantly from those in lithotripsy, but have similar amplitudes. A fiber-optical probe hydrophone provides the possibility of minimal invasive pressure measurements in vivo because of its small sensor diameter. During insonification in rabbits, the focal sound pressures and spatial pressure distributions were recorded in the liver and the back muscle in vivo. The fiber tip of the hydrophone was placed directly in the tissue by puncturing, using a cannula. The results obtained are compared with in vitro measurements. They are discussed regarding the influence of attenuation and nonlinear propagation in tissue. Additionally, an error estimation for the pressure measurement in vivo is presented. [Work supported by Wilhelm Sanders Stiftung Gz. 89.030.2.] 


\section{4:40}

4pPAb8. Fiber-optic dielectric multilayer hydrophone for ultrasound detection. Volker Wilkens and Christian Koch (Lab. 1.43, Physikalisch-Technische Bundesanstalt, PF 3345, D-38023 Braunschweig, Germany)

Fiber-tip sensors have proved to be useful for the investigation of ultrasonic signals since they, in principle, overcome some limitations of common piezoelectric hydrophones. A high damage threshold of the sensor is obtained, measurements are not disturbed by electromagnetic influences, and a high temporal and lateral resolution is possible. An optical hydrophone is presented that comprises a fiber tip coated with a dielectric multilayer system of the Fabry-Perot type. The acoustic pressure measurement using the sensor is based on the elastic deformation of the layer system by an incident ultrasonic wave and the detection of the induced change of the optical reflectance. Measurement results obtained by sensors with different layer numbers providing different sensitivities are shown, and the variation of the sensor characteristics depending on the size and shape of the fiber tip is investigated. The appearance of optical loss modulations caused by the stimulation of flexural waves in the fiber in certain measurement configurations is discussed. Several sensor designs are considered to improve the performance of the system in different fields of application, for instance the characterization of lithotripter shock waves,
5:00

4pPAb9. Ultrasound cleaning of microfilters. Jens Hald, Irina Bjørnd (UltraTech ApS, Målbv Byvej 229, DK-2760, Målbv, Denmark), and Leif Bjørnd (Tech. Univ. of Denmark, DK-2800 Lyngby, Denmark)

The aim of the present work is to develop, design, and manufacture a high-power ultrasound transducer module to be used for preventing the blocking of plastic-based microfilters by organic materials, and possibly to prolong the lifetime of the filters in industry using the cavitation on the surface of the filter. A numerical, FE- and BE-based model for calculation of the response of ultrasonic transducers of various geometries formed the basis for the design of such transducers. During laboratory experiments frequency and output power have been varied in order to find the optimal transducer design suitable for cleaning of microfilters without damaging the filter structure. The filter surface was studied using an optical microscope before and after the experiment. When high-power ultrasound (max. $75 \mathrm{~W} / \mathrm{cm}^{2}$ ) was applied to the surface of some microfilters, no visible damage was found, while others filters were damaged. The results of the laboratory experiments formed background for the final design of an ultrasound transducer module for use by foodstuff filtration plants. [This work was financed by the EU Project WAMBIO PL96-3257 (FAIR Programme).]

\section{Contributed Posters}

These posters will be on display in the Poster Gallery from Thursday to Friday, 18-19 March. Authors will be at their posters from 5:20 p.m. to 6:20 p.m. on Thursday, 18 March.

4pPAb10. Laser Doppler anemometry applied to airborne acoustics: Influence of the signal processing parameters. Sylvain Poggi, Bruno Gazengel, Jean-Christophe Valière, and Anne-Marie Bruneau (IAM, Laboratoire d'Acoustique de l'Université du Maine, UMR CNRS 6613, Av. Olivier Messiaen, F-72085 Le Mans Cedex 9, France)

Laser Doppler anemometry (LDA) is widely used for fluid mechanics but may also provide measurements of the acoustic particle velocity. The validation of its use in acoustics, which is being achieved, requires knowledge of the appropriate data processing parameters. In order to understand their effects, an experiment is conducted in which LDA measures structural velocities. In this experiment, the velocity of a shaker tip measured by LDA is compared with the velocity measured by a laser vibrometer. The LDA signal is a sinusoidal frequency modulation whose instantaneous frequency is proportional to the measured velocity. LDA-measured velocities are estimated using either spectrum analysis or time-frequency analysis. These comparisons point out three major results. Even if the velocity is small, which corresponds to a narrow bandwidth of the measured signal, oversampling should be preferred. Time windows should be chosen as short as possible despite the poor estimation of the instantaneous frequency. These first two results lead to a precise temporal description of the measured velocity but a rough one in the amplitude domain. However, the a priori knowledge of the excitation signal frequency enables the precise estimation of the velocity amplitude by means of a least-square method.

4pPAb11. Acoustoelectric effect in gas sensor of the SAW type. Marian Urbanczyk and Aleksander Opilski (Inst. of Phys., Tech. Univ. of Silesia, ul. B. Krzywoustego 2, 44-100 Gliwice, Poland)

The initial value of the electric conductivity of the sensorial layer determines the working point of the sensor and is of essential importance with respect to the sensitivity of the sensor, because it determines the value of the acoustoelectric effect in that system. Basing on measurements it has been found that the electric conductivity of a lead phthalocyanine layer placed in an $\mathrm{NO}_{2}$ atmosphere increases. This effect becomes higher as the temperature rises. In a SAW sensor the effect of the electric potential of a surface wave with a sensorial layer occurs in a high-frequency field. It has been found that the conductivity of lead phthalocyanine layers depends on the frequency to an extremely high degree. If compared with direct-current measurements, the alternating-current conductivity is greater by about two orders; its frequency being equal to the frequency of the propagated surface wave applied $(43 \mathrm{MHz})$. Practically, the value of the conductivity of semiconducting layers at higher frequencies makes possible the occurrence of an acoustoelectric effect in the sensor.

4pPAb12. SAW sensor for low nitrogen dioxide concentration. Aleksander Opilski, Marian Urbanczyk, and Wiesaw Jakubik (Inst. of Phys., Tech. Univ. of Silesia, ul. B. Krzywoustego 2, 44-100 Gliwice, Poland)

The devolopment of a technique concerning macromolecular compounds has made it possible to get thin layers that change their physicochemical properties as a result of their interaction with the ambient atmosphere. Changes of the physical properties of specially prepared chemically sensitive layers influence the conditions of the propagation of an acoustic surface wave arranged in the layered system piezoelectric waveguide layer of the compound. Particularly, if the mass and electric conductivity of the chemically sensitive layer changed, the velocity of propagation of the acoustic wave changes. The presented paper includes a short review of the SAW gas sensor field and some experimental results for the detection of a low concentration of nitrogen dioxide in the air. 


\title{
Session 4pPAc
}

\section{Physical Acoustics: Elastic Properties of Materials II: Special Materials}

\author{
Jan D. Achenbach, Cochair \\ Center for Quality Engineering and Failure Prevention, Northwestern University, 2137 North Sheridan Road, Evanston, \\ Illinois 60208-3020, USA \\ Heinz-Jürgen Fröhlich, Cochair \\ Paul-Drude Institut für Festkörper-Elektronik, Hausvogteiplatz 5-7, D-10117 Berlin, Germany
}

Invited Papers

2:00

4pPAc1. Effects of grain size and shape on ultrasonic attenuation and backscattering. R. B. Thompson (Ctr. for Nondestruct. Eval., Iowa State Univ., Appl. Sci. Complex II, 1915 Scholl Rd., Ames, IA 50011)

The influence of scattering from gain boundaries on the attenuation and backscattering of ultrasonic waves in polycrystals is a classical problem which has received considerable attention. However, the great majority of the effort has considered equiaxed grains. Extensions to polycrystals with elongated grains and multiple dimension scales will be presented. Experimental results will be reported for aluminum and titanium polycrystals, including a number of cases in which the directions of high attenuation exhibit low backscattering and vice versa. Although these observations were initially found to be surprising, significant progress has been made towards an understanding. The status of diagnostic experiments and theoretical efforts to explain these observations will be summarized. Implications of the results in the design of improved nondestructive techniques to detect flaws and characterize microstructure, including progress towards in situ techniques to monitor recrystallization will be discussed. [Portions of this work supported by the United States DOE, NSF, and the FAA.]

\section{2:20}

4pPAc2. Ultrasonic study of multi-ply composites. S. I. Rokhlin (Ohio State Univ., Nondestruct. Eval. Prog., Edison Joining Technol. Ctr., 1248 Arthur E. Adams Dr., Columbus, OH 43221)

Multi-ply composites are studied ultrasonically using transmission and reflection spectroscopy and time-resolved line focus acoustic microscopy. For quasi-isotropic multi-ply composites, the ultrasonic response does not exhibit isotropic behavior as, for example, is observed in mechanical tests. The amplitudes of the ultrasonic obliquely transmitted/reflected signals depend on the sample orientation relative to the incident plane as well as top ply orientation and ply sequence. In addition to the plane-wave study, the applicability of the time-resolved line focus acoustic microscopy for determination of elastic constants of composites is discussed and the results are compared to data obtained by the self-reference double-through-transmission ultrasonic bulk wave method. A theoretical model to predict ultrasonic wave propagation in the multi-ply anisotropic structure is described. The efficiency and performance of the transfer and global matrix methods are compared. The theoretical results are compared with experiment and used to interpret the complicated wave behavior in this material.

\section{2:40}

4pPAc3. Integration of modeling and acoustic microscopy measurements for multiple layered coatings. J. D. Achenbach and Z. Guo (Northwestern Univ., Ctr. for Quality Eng. and Failure Prevention, 2137 Sheridan Rd., Evanston, IL 60208, achenbach@nwu.edu)

The determination of material constants of multiple-layered coatings by line-focus acoustic microscopy requires a combination of the $V(z)$ measurement technique with a well-founded and accurate measurement model. This paper is concerned with the determination of the material constants of a two-layered $\mathrm{ZrC} / \mathrm{ZrN}$ coating on a stainless steel substrate, as well as with an evaluation of the bond quality of the interfaces in-between the layers. A $V(z)$ measurement model, which accounts for bond-quality variations, is developed by combining a derived spectrum of reflection coefficients with acoustic microscopy considerations. Material constants are determined by systematically minimizing the differences between measured results, obtained by line-focus acoustic microscopy, and results simulated with the measurement model. Sensitivity of the technique and accuracy of the results are briefly discussed. 


\section{Contributed Papers}

\section{3:00}

4pPAc4. Review of the method of reduced variables for the dynamic mechanical properties of viscoelastic materials. Bruce Hartmann and Gilbert F. Lee (Carderock Div. Naval Surface Warfare Ctr., West Bethesda, MD 20817-5700, hartmann@dt.navy.mil)

Under certain conditions, a change in temperature is equivalent to a change in frequency for dynamic mechanical measurements in viscoelastic materials. This property allows one to extend the effective frequency range of the measurements by shifting isotherms along the log frequency axis using the method of reduced variables, also known as timetemperature superposition. The most commonly used analytical equations describing the required shift are the WLF equation and the Arrhenius equation, but shifting can be implemented without the necessity of an analytical representation. This review will consider the physical basis for this property, known as thermorheological simplicity, the limitations of its applicability, and the advantages and disadvantages of various shifting procedures. Techniques that have been used include shifting the real part of the modulus, the imaginary part of the modulus, the loss factor, and the use of complex compliance rather than complex modulus. Other important issues are the choice of reference temperature and the use of a vertical shift in addition to a horizontal shift. [Work supported by the Carderock Division of the Naval Surface Warface Center's In-house Laboratory Independent Research Program sponsored by the Office of Naval Research.]

\section{3:20}

4pPAc5. Ultrasonic anomalies in betaine phosphate/betaine phosphite mixed crystals. Juras Banys, Vytautas Samulionis (Faculty of Phys., Vilnius Univ., Sauletekio 9, Vilnius 2040, Lithuania, juras.banys@ff.vu.lt), Georg Voelkel (Univ. Leipzig, Leipzig D-04103, Germany), and A. Kloepperpieper (Univ. des Saarlandes, D-66123, Sarrbruecken, Germany)

Measurements of the longitudinal ultrasonic velocity and the attenuation along the $x, y$, and $z$ axes have been carried out in deuterated betaine phosphite (DBPI) single crystals in the vicinity of the ferroelectric and antiferrodistortive phase transitions. For ultrasonic modes propagating along the $x$ and $z$ axes the critical slowing down has been observed, but along the $y$ axis critical slowing down was absent for both phase transitions. Regardless of the weakness of elastic anomalies at ferroelectric phase transition the ultrasonic behavior in DBPI can be explained by the Landau-Khalatnikov theory for uniaxial ferroelectrics. The ultrasonic behavior at the antiferrodistortive phase transition indicates that this phase transition is very close to the tricritical one. The relaxation time of the order parameter in the vicinity of the transition to the antiferodistortive phase for DBPI crystal was estimated. Similar results have been obtained for the mixed crystals betaine phosphate/betaine phosphite in the region of the antiferrodistortive phase transition. The temperature behavior of the ultrasound velocity and attenuation indicates the existence of the ionic conductivity in these cystals. It should be noted that conductivity phenomena is highly anisotropic in these crystals.

\section{3:40-4:00 Break}

\section{4:00}

4pPAc6. Ultrasonic absorption in concrete. Joseph A. Turner (Dept. of Eng. Mech., W317.4 Nebraska Hall, Univ. of Nebraska-Lincoln, Lincoln, NE 68588-0526, jat@unlinfo.unl.edu)

The propagation of ultrasound in concrete is greatly affected by the heterogeneous composition. An ultrasonic plane wave will attenuate as it propagates. Part of this attenuation is due to scattering effects which result from the aggregate. Another component of the attenuation is due to true dissipation from viscoelastic effects. Total attenuation has been previously proposed as a materials characterization parameter, although the distinction between scattering attenuation and dissipation is often not made. Experimental results are presented which should help separate these effects.
Absorption measurements were made on a number of specimens with differing aggregate composition and water-cement ratio. The absorption is shown to be independent of the nature of the aggregate, as expected. In addition, the absorption is shown to be dependent upon the water-cement ratio and thus is related to the strength. Frequency dependence of absorption is also discussed. It is anticipated that ultrasonic absorption may be a useful parameter for characterizing concrete material properties. [Work supported by the Nebraska Research Initiative and Layman Fund.]

\section{$4: 20$}

4pPAc7. Elastic wave propagation and scattering in heterogeneous, anisotropic media: Textured polycrystalline materials. Joseph A. Turner (Dept. of Eng. Mech., W317.4 Nebraska Hall, Univ. of NebraskaLincoln, Lincoln, NE 68588-0526, jat@unlinfo.unl.edu)

The propagation of elastic waves through heterogeneous anisotropic media is considered. Appropriate ensemble averaging of the elastic wave equation leads to the Dyson equation which governs the mean response of the field. The Dyson equation is given here in terms of anisotropic elastic Green's dyads for the medium with and without heterogeneities. The solution of the Dyson equation for the mean response is given for heterogeneities that are weak. The formalism is further specified for the case of equiaxed cubic polycrystalline metals with a single aligned axis. The Green's dyads in this case are those for a transversely isotropic medium. Simple expressions for the attenuations of the shear horizontal, quasicompressional, and quasishear waves are given in terms of integrations on the unit circle. The derived expressions are limited to frequencies below the geometric optics limit, but give the attenuations in a direct manner. Comparisons with previous results are also discussed. It is anticipated that a similar approach is necessary for the study of wave propagation in complex anisotropic materials such as fiber-reinforced composites. In addition, the results are applicable to diffuse ultrasonic inspection of textured polycrystalline media.

\section{$4: 40$}

4pPAc8. Elastic modules modification and phonon focusing in zinc oxide. Uladzimir V. Zubrytski (Inst. of Phys., Natl. Belarus Acad. of Sci., 68 Scaryna Ave., Minsk 220072, Belarus)

Despite the direct observations of the acoustic waves focusing in different solids [J. P. Wolfe, Phys. Today 48, 34-40 (1995)], the properties of the phonons' propagation and possibility of these control have not been studied in hexagonal media. To check this statement [U. V. Zubrytski, Z. Tekhn. Fiz. 67, 59-64 (1997)] and obtain striking demonstrations of drastic changes in the phonon focusing patterns at elastic properties, modification of such solids' phonon focusing is simulated in zinc oxide grown by the hydrothermal method, in the vapor state at and without doping with lithium, and magnetron sputtering, in this paper. Simulation shows that elastic modules modification within the accuracy of available experimental data causes both sharp increasing of the intensities and defocusing of the individual phonon modes. Quantitative dependences of the effect have been established for bulk acoustic waves in the samples investigated. The findings allow one to elaborate not only the equilibrium but the dynamic method to control selectively both intensities and angular distributions of the acoustic energy fluxes in the crystals and thin layers as well. Besides, the elastic constants recovering from the measuring of the focusing factor can be realized. [Work partially supported by the Belarusian Republican Foundation for Fundamental Research.]

\section{5:00}

4pPAc9. Picosecond ultrasonics in gold nanoparticles. Arnaud Devos (IEMN, Dept. ISEN, 41 Bd. Vauban, 59046 Lille, France), Bernard Perrin, and Jean Claude Jeannet (Univ. Pierre et Marie Curie, CNRS, 75252 Paris Cedex 05, France)

Pump-probe optical techniques with femtosecond laser sources can be used to provide information about the acoustical properties of very thin films. With this so-called "picosecond ultrasonics technique," echoes shorter than 10 ps can be time-resolved in films whose thicknesses are 
larger than a few tens of $\mathrm{nm}$; for smaller thicknesses, the acoustic excitation induces very high frequency acoustic resonances ranging from 0.1 up to $2.5 \mathrm{THz}$. In this work, the results of a picosecond ultrasonics experiment performed on gold colloids which represent an example of nanostructures is presented. The samples studied were of three types: solutions (gold particles dispersed in water), gels (gold particles embedded in a polymer matrix), and a film of particles attached on a polymer monolayer. The particle size was between 5 and 20 nanometers. In all the samples, oscillations in the transmitted probe beam which are ascribed to the vibrational mode of the sphere were observed. A second oscillation with a much longer period is also reported in the solution and gels cases. [Work supported by CNRS.]

\section{$5: 20$}

4pPAc10. Ultrasonic waves birefringence in wood-based composite panels. Voichita Bucur (Université "Henri Poincaré'” Nancy I, BP 239, F-54506 Vandoeuvre, France, bucur@lermab.u-nancy.fr) and Simone Garros (Ctr. de Recherches Forestières Nancy, F-54280 Champenoux, France)

The aim of this research is to study the ultrasonic waves birefringence in three wood-based composite panels, chipboards, oriented strandboards, and medium-density fiberboards, for the characterization of their global elastic and structural properties. Shear waves of $1 \mathrm{MHz}$ were used to study the ultrasonic birefringence and to determine the shear terms of the stiffness matrix. As a complement for anisotropic behavior panel study, longitudinal waves of the same frequency were used to determine the corresponding terms of the stiffness matrix. Shear wave birefringence is the highest in the transversal anisotropic plane of the panels. Textural anisotropy was observed at the scale of the skin and core. Data related to the local characterization of the skin and core of the panels by $\mathrm{x}$-ray mi- crodensitometric technique revealed that, generally, the profile of the panel is symmetric and the skin density is higher than the core density. The nondestructive estimation of the birefringence of ultrasonic waves is a useful tool for further evaluation of panel performance related to their structural integrity and bond performance.

\section{5:40}

4pPAc11. Investigation of dissipative effects in a poroelastic layer coated plate. Stephane Rigobert, Franck Sgard (Lab. des sciences de 1'habitat, DGCB, URA CNRS 1652, Ecole Nationale des Travaux publics de l'Etat, 2 rue Maurice Audin, 69518 Vaulx-en-Velin, France), and Noureddine Atalla (Univ. de Sherbrooke, QC J1K 2R1, Canada)

Nowadays, porous media are widely used in multilayered structures for noise control applications in such fields as aeronautics, automobiles, building acoustics, and environmental acoustics. Recently, a finite-element model based on pressure-deplacement formulation of Biot's poroelasticity theory has been extensively used to predict the acoustical and structural reponse of a multilayer structure. For practical applications, such models may lead to large frequency-dependent matrices and thus require huge computational ressources. The goal of this work is to solve the problem in the particular case of a poroelastic layer attached to an elastic plate. The idea is to develop a plate finite element which acounts for the behavior of the elastic plate and the porous solid phase. The fluid phase of the porous medium will be taken into account using equivalent fluid finite elements. In this paper, the assumptions and numerical results justifying the proposed simplifications will be discussed. In particular, the relative importance of the different dissipation mechanisms in the plate-porous system will be numerically assessed. These results constitute guidelines for considering the relevant dissipation phenomena in the simplified finiteelement model of the system.

THURSDAY AFTERNOON, 18 MARCH 1999

ROOM H107, 2:00 TO 4:00 P.M.

\title{
Session 4pPPa
}

\section{Psychological and Physiological Acoustics: Hearing Impairment, Hearing Instruments and Cochlear Implants}

\author{
Astrid van Wieringen, Chair \\ Laboratory Experimentele ORL, KU Leuven, Kapucijnenvoer 33, 3000 Leuven, Belgium
}

Contributed Papers

\section{2:00}

4pPPa1. Contribution of reduced audibility to subnormal localization performance in hearing-impaired listeners. S. Theo Goverts, Tammo Houtgast, and Sophia E. Kramer (Dept. of Otorhinolaryngology/Audiol. Hospital, Vrije Universiteit, P.O. Box 7057, 1007 MB Amsterdam, The Netherlands, st.goverts@azvu.nl)

After speech reception, sound localization seems to be the secondmost important hearing problem associated with auditory impairment. Recently a laboratory test on localization was developed which provides good resemblance to localization tasks in daily life. Subjects are presented with everyday sounds (about $1.5 \mathrm{~s}$ each) from eight evenly distributed directions. Only on the target sound (a telephone), presented randomly in time, were listeners required to indicate the azimuth of presentation. Data from 40 hearing-impaired listeners show a large variability in performance ranging from near normal to very poor. Aspects of hearing that may contribute to a subnormal performance are reduced audibility, incomplete or noisy spectro-temporal coding in the auditory periphery, or inadequate binaural processing. As a first step towards understanding the reduced performance for hearing-impaired listeners, results will be discussed on a localization test for normal-hearing listeners with noise-masked audiograms that match the audiograms for categories of hearing-impaired listeners. Results will be compared to previous findings on the role of audibility and spectro-temporal coding in the precedence effect.

\section{2:20}

4pPPa2. Method of measuring and preventing unstable feedback in hearing aids. Shawn X. Gao and Sigfrid D. Soli (House Ear Inst., Los Angeles, CA 90057)

Acoustic feedback is a practical problem that can limit the performance and benefit of any hearing aid. In the past, there have been no clinical available methods for accurately predicting the frequencies and gains that will cause unstable feedback for a particular hearing aid in an 
individual's ear. The proposed method uses a typical clinical real-ear probe microphone system to acquire the real-ear aided responses by placing the hearing aid in the ear canal and setting the hearing aid gain to two different known settings. The Open Loop Transfer Function (OLTF) of the hearing aid can be derived based on the acquired aided signals. Once the OLTF of the hearing aid is known, potentially unstable frequencies are identified and maximum hearing aid gain and maximum hearing aid insertion gain are determined. The measurement allows the dispenser to modify the desired hearing aid response at only the frequencies that will cause unstable feedback. It consequently eliminates the trial and error method and prevents over-adjustment of the hearing aid. A description of theory and measurement procedure will be provided, as well as examples of its use on KEMAR and hearing-impaired individuals.

\section{2:40}

4pPPa3. Artificial noise signals with speechlike spectral and temporal properties for hearing instrument assessment. Wouter A. Dreschler (Dept. of Exp. and Clinical Audiol., Academic Medical Ctr., P.O. Box 22660, NL-1100 DD, Amsterdam, The Netherlands, w.a.dreschler@amc.uva.nl), Hans Verschuure (Erasmus Univ., Rotterdam, The Netherlands), Carl Ludvigson, and Soren Westermann (Topholm \& Westermann, DK-3500 Vaerlose, Denmark)

The introduction of different forms of nonlinear amplification and noise-reduction algorithms calls for more intelligent ways to specify the properties of hearing instruments. Traditional specifications no longer reflect important characteristics of hearing instruments. Also, for the psychophysical evaluation in a laboratory setting, well-defined noises are necessary; aided speech perception will be largely determined by the properties of the speech signal and the concurrent background noise. The field of hearing instrument specification and evaluation has become a complex area in which traditional tools have lost their relevance. There is a strong need for a new approach based on new test signals that are broadband and dynamic. Standardized broadband noises are required for comparative measurements in different hearing instruments. Based on these notions a working group of the International Collegium of Rehabilitative Audiology (ICRA) started a consensus process to design well-defined artificial noise signals that can become a standard for hearing instrument assessment, both for technical measurements and for applications in the clinical and psychophysical evaluation. This paper reports about the design criteria, the actual realization, and some examples of applications in the measurement of digital signal processing hearing instruments.

\section{3:00}

4pPPa4. Gap detection thresholds of Laura cochlear implant users for complex patterns of stimulation. Astrid van Wieringen and Jan Wouters (Lab. Experimentele ORL, KULeuven, Kapucijnenvoer 33, 3000 Leuven, Belgium)

The ability to detect silent intervals on single and multiple electrode pairs was examined for different types of speechlike stimulus configurations in four postlingually deafened Laura cochlear implantees. Gap detection thresholds were determined in function of stimulus complexity, spectral disparity within and across the markers bounding the gap, stimulus asymmetry (cf. consonant-vowel versus vowel-consonant patterns), and pulse rate. All stimuli were roving in duration from 200 to $600 \mathrm{~ms}$ to ensure that subjects could not use overall duration as a cue. In addition, all the experimental conditions were balanced in loudness. Preliminary results have shown that stimulation of single electrode pairs yields smaller gap detection thresholds than stimulation of multiple electrode pairs, and that gap detection thresholds on multiple electrode pairs increase as the distance between the electrodes increases. Subsequent measurements illustrate that gap detection thresholds across multiple electrode pairs can decrease substantially after sufficient training, and that the spectral composition of the stimulus only affects gap detection when the spectral disparity occurs across, not within, markers. These findings, together with others concerning stimulus asymmetry and pulse rate, are of fundamental importance to understand the biophysical limitations of electrical stimulation related to the coding of sound by the auditory system.

\section{3:20}

4pPPa5. Two-microphone noise reduction for the LAURA cochlear implant. Jeff Vanden Berghe and Jan Wouters (Lab. Experimentele ORL, KULeuven, Kapucijnenvoer 33, 3000 Leuven, Belgium)

An adaptive noise cancellation scheme based on two-stage adaptive filtering has been developed for a two-microphone configuration in one hearing aid. This strategy has been tested with hearing-impaired listeners and yields a significant improvement of the speech reception threshold in background noise, as measured with sentences and two-syllable words [J. Vanden Berghe and J. Wouters, J. Acoust. Soc. Am. 103, 3621-3626 (1998)]. As the effect of background sounds is even more severe for cochlear implant (CI) users than for the hearing impaired in general, this strategy was tested with four LAURA CI users. The system was implemented in real time on Audallion, a portable DSP-platform for hearing aids (Audiologic-Resound-Danavox Digital Alliance), and used as a preprocessor for the input to the CI speech processor. Speech intelligibility data in noise were obtained with and without this two-microphone noise reduction algorithm. The data indicate a similar effect for CIs as for hearing-impaired and normal-hearing people. For all subjects, measurement of the SRT for the two-stage adaptive filtering strategy shows an improvement of more than $10 \mathrm{~dB}$ in SNR when compared to the unprocessed condition. An analysis of variance indicates this improvement to be highly significant.

\section{$3: 40$}

4pPPa6. Efficiency of a medical treatment of the acoustic trauma following impulse noise exposure in the guinea pig. Pascal Magnan, Christine d'Aldin, and Armand L. Dancer (French-German Res. Inst. of Saint-Louis, 5 rue du Général Cassagnou, BP 34, F-68301 Saint-Louis Cedex, France, dancer@newel.net)

Most of the patients suffering from acute acoustic trauma have been exposed to impulse noises. Temporary or permanent threshold shift (TTS or PTS) can be observed after such an exposure. They are correlated to structural and functional modifications of the peripheral auditory system and particularly of the sensory hair cells. Studies performed in guinea pig exposed to continuous noise have shown a positive effect of corticotherapy and oxygenotherapy (the treatments are given immediately after the end of the exposure). The aim of this paper is to assess the efficiency of those treatments in the guinea pig exposed to impulse noises; i.e., 20 pistol rounds of 157-dB peak pressure (at a 5-s interval). Threshold shifts (electrocochleography) and distortion products are measured for 15 days and are compared with histological observations (scanning electron microscopy). The results allow one to understand better the cochlear mechanisms of damages and to assess the efficiency of the classical treatments of the acoustic trauma following impulse noise exposures. 


\title{
Session $4 p P P b$
}

\section{Psychological and Physiological Acoustics: Human Factors (Poster Session)}

\author{
Volker Hohmann, Chair \\ AG Medizinsche Physik, Universität Oldenburg, D-26111 Oldenburg, Germany
}

\section{Contributed Papers}

\begin{abstract}
All posters will be on display from Thursday to Friday, 18-19 March. Contributors will be at their posters from 4:00 p.m. to 6:00 p.m. on Thursday, 18 March.
\end{abstract}

\begin{abstract}
4pPPb1. The detection of complex submarine signals: A study of the linear frequency modulation criterium. François Santon and Agnes Tozza (LMA-CNRS, $31 \mathrm{Ch}$ J. Aiguier, 13402 Marseille Cedex 20, France, santon@1ma cnrs-mrs.fr)

The objective of this work is to know, in the context of submarine detection, what is the effect of a linear frequency modulation on the detection of glissando's signals. Indeed, this frequency modulation is often present in submarine signals, whether of biological or mechanical origin. Different types of glissandos are considered, increasing, increasingdecreasing, increasing with plateau, and decreasing. Differences have not been noted between the detection thresholds for the first three types of signals. In general, the detection threshold is very close to the maximum frequency of the signals threshold. In the case of a decreasing glissando, it can be noticed that the detection threshold is lower by a few $\mathrm{dB}$ than the threshold of the signal's highest frequency.
\end{abstract}

4pPPb2. Construction of a minimal set of sound parameters for multidimensional tests. Etienne Parizet (Renault, 67 rue des bons raisins, 92508 Rueil-Malmaison Cedex 9, France) and Valery Nosulenko (Anvie, Paris, France)

In a multidimensional test, the selection of sound parameters may be difficult, because these parameters must not be numerous while describing the whole perception and have to be understood unambiguously by naive listeners. The method of free verbalizations [E. Samoylenko, S. McAdams, and V. Nosulenko, Int. J. Psych. 31(6), 255-278 (1996)] allows the construction of a minimal set of parameters. A minimal set of seven parameters was built for diesel engine idle noise. These parameters were then used for a semantic differential test, with other listeners and no sound training session. It was checked that the characterizations of sounds thus obtained for each parameter were very close to those given by the first test using free verbalizations. That means that the parameters were well understood by listeners and that all semantic ambiguities were avoided. Such a parameter set can be used for routine tests in the same sound context.

4pPPb3. Sound and vibration at low frequencies. Michael A. Bellmann, Carsten Reckhardt, Volker Mellert (Physik Akustik, Carl v. Ossietzky Univ. Oldenburg, D-26111, Germany), and Hermann Remmers (Inst. für Technische und Angewandte Physik GmbH, Oldenburg D-26111, Germany)

Equal-loudness-level contours are assumed to reflect the frequency sensitivity of the auditory system. Studies on equal-loudness-level contours show considerable differences even when the same measurement method is used. Recent studies [Gabriel et al., DAGA 96; Reckhardt et al., DAGA 97] report on the influence of measurement method, thresh- old, and choice of test tone level. In this study, an adaptive 2-AFC method [Reckhardt et al., DAGA 97; Verhey et al., DAGA 97] is used for measuring equal-loudness level contours in the frequency range of 16 to 160 $\mathrm{Hz}$ at 60 phon. The data are measured in a pressure chamber for 12 normal-hearing subjects. Results are higher than the standard curve specified in ISO 226. In a study of Reckhardt et al. it is shown that the interindividual variance is decreased by taking the individual threshold of hearing into account. The results from low-frequency measurements support this finding. In addition, this study investigates the influence of vibration on loudness perception. Equal-loudness-level contours are measured in the presence of distinct vibration levels above vibration threshold in a frequency range of 10 to $80 \mathrm{~Hz}$.

4pPPb4. Development and evaluation of an adaptive loudness scaling procedure. Thomas Brand and Volker Hohmann (AG Medizinische Physik, Universität Oldenburg, D-26111 Oldenburg, Germany, tom@medi.physik.uni-oldenburg.de)

Loudness scaling procedures are used in the diagnostics of hearing impairment to assess the recruitment phenomenon, i.e., the pathological growth of loudness. In these procedures, acoustical stimuli at different levels are presented to the subject and the task is to rate the perceived loudness on a category scale. The aim of this study is the development and evaluation of an adaptive loudness scaling procedure in which the level of the next stimulus is calculated on the basis of the previous trials. Based on empirical results from normal-hearing and hearing-impaired listeners, the procedure was optimized in order to yield minimal test-retest deviations and minimal biases due to range and context effects at minimum measuring time. To achieve these aims, different settings of the following parameters were tested: Number of response alternatives, track length, initial level, adaptive procedure, and parametrization of the loudness function. The best setting was shown to yield an equal distribution of stimulus levels in the full dynamic range of the individual subject with a minimum number of "inaudible", and "too loud" presentations. For each categorial loudness the corresponding stimulus level is estimated with a standard deviation of about $5 \mathrm{~dB}$.

4pPPb5. Factors influencing the subjective rating of tonal components in noise. Matthias Vormann (Carl von Ossietzky Universität Oldenburg, Fachbereich Physik Arbeitsgruppe Akustik, Carl von Ossietzky Strasse 9-11, D-26111 Oldenburg, Germany, matthias@aku.physik.uni-oldenburg.de) and Jesko L. Verhey (Carl von Ossietzky Universität Oldenburg, D-26111 Oldenburg, Germany)

If environmental or artificial noises contain tonal components, they are more annoying than noises without tonal components. Therefore the German norm, DIN 45681 (draft): "Detection of tonal components of noise and determination of a tone adjustment for the assessment of noise immi- 
sions" was proposed to quantify this increased annoyance. Within this definition the effects of the tonal components are reduced to the increasing annoyance of the signals. In the literature no satisfying data exists about the subjective ratings of tonal components in noises (in German called "'Tonhaltigkeit"). For this reason the present study investigates the influence of selected physical parameters on subjective ratings of the "Tonhaltigkeit." An adaptive procedure is used to adjust the hearing impression "Tonhaltigkeit" of different noises to a reference signal consisting of a single sinusoidal.

4pPPb6. Effects of a neoprene wetsuit hood on low-frequency underwater hearing thresholds. John R. SimsII, David M. Fothergill, and Michael D. Curley (Naval Submarine Medical Res. Lab., Box 900, Groton, CT 06349-5900)

In an effort to create guidelines for exposure to low-frequency underwater sound, it is necessary to obtain population data on the minimal detectable sound-pressure level at these frequencies. Underwater hearing thresholds were obtained from 15 male and 5 female recreational SCUBA divers with and without a $3-\mathrm{mm}$ wetsuit hood. Dives were conducted at a $1-\mathrm{m}$ depth in a large quiet anechoic pool. A J13 transducer was located 4 $\mathrm{m}$ directly below the subject. Thresholds were determined using twointerval forced-choice with a 0.71 probability of positive response at convergence. A 1 -s pure tone was presented with a 20 -ms rise and fall at 100 , $200,250,300,400$, and $500 \mathrm{~Hz}$. Results indicate there was no difference in thresholds between male and female divers. Without a wetsuit hood, mean thresholds decreased from $99 \mathrm{~dB}$ (s.d. $6 \mathrm{~dB}$ ) re: $0.000001 \mathrm{~Pa}$ at 100 $\mathrm{Hz}$ to $85 \mathrm{~dB}$ (s.d. $10 \mathrm{~dB}$ ) at $500 \mathrm{~Hz}$. Thresholds were statistically similar at 100 to $300 \mathrm{~Hz}$ with and without the wetsuit hood, but were significantly increased at 400 and $500 \mathrm{~Hz}$ with the hood $(p<0.001)$. In conclusion, a wetsuit hood attenuates frequencies at $400 \mathrm{~Hz}$ and above by approximately $10 \mathrm{~dB}$. [Work supported by CNO, N-87.]

4pPPb7. Estimates of the instantaneous versus overall loudness of noise emissions. Thomas Filippou and Hugo Fastl (Inst. of Man-Machine-Commun., Tech. Univ. Muenchen, Arcisstr. 21, D-80333 Muenchen, Germany)

Usually when comparing the relations between instantaneous and overall loudness judgments of noise emmissions, the overall loudness is larger than the average of the instantaneous loudness judgments. Obviously, the prominent parts of a noise emmission play a crucial part in overall loudness judgment. Two models were proposed to describe the overall loudness on the basis of the instantaneous loudness judgments [Fastl, DAGA 97]. The merits of the different models in predicting the overall loudness of noise emissions with a different number of events were studied. For emissions with 2 up to 11 events within $15 \mathrm{~min}$, a simple percentile of the instantaneous loudness judgments cannot predict overall loudness. However, when the events are prolonged by an exponential decay with 30-s time constant, the judgment of overall loudness can be predicted with an average accuracy of $6 \%$.

4pPPb8. On the use of auditory information in human movement control. Andreas Hellmann and Rainer Guski (Univ. Bochum, Dept. of Psych., D-44780 Bochum, Germany, hellmann@psychologie.uni-oldenburg.de)

A human acting and moving in the environment often produces sounds, which are related to his or her body movements. Movement control requires the continuous use of perceptual information about the ongoing movement. It was investigated whether humans can and do use sound produced by their movements as auditory information for the control of their movements. In the experiments the movements or tasks required from the subjects were varied, as well as the available auditory and visual information. The success in the movement tasks was measured. The results obtained so far show that auditory information is used for movement control by the subjects, especially with complicated movements.

4pPPb9. Short and medium motor responses to auditory pitch shift: Latency measurements of the professional musician's audio-motor loop for intonation. Dietrich Parlitz and Marc Bangert (Inst. for Musicphysiol. and Performing Arts Medicine, Univ. for Music and Theatre, D-30175 Hannover, Germany, parlitz@hmt-hannover.de)

For the singer's voice, as well as for many musical instruments, like strings or brass, a precise intonation relies on a quick and adaptive finetuning mechanism, i.e., a motor response correcting for deviations of the produced tone due to mechanical properties of the body and instrument as well as due to environmental parameters. The aim of the procedure is either to be in tune with an internal representation of the correct pitch or to be in harmony with accompanying instruments or singers. In an experimental setup with a real-time audio pitch shifter and an infrared-based motion analysis system, this unique ability of musicians can be studied systematically. In a group of (a) expert viola players, (b) expert trombone players, and (c) professional singers, the distribution of pitch-shiftreaction-times (PSRTs) is measured (1) in a solo-player/singer situation and (2) in an ensemble/choir situation. Results are compared to recent studies with nonmusicians [T. A. Burnett et al., J. Acoust. Soc. Am. 103, 3153-3161 (1998)]. Similarities to other orientation reactions like saccadic eye-movements and to the rapid pitch-correcting mechanisms in the echo-locating system of the bat are discussed. 


\title{
Session 4pSAa
}

\section{Structural Acoustics and Vibration: Smart Materials II}

\author{
Sabih I. Hayek, Cochair \\ Department of Engineering Science and Mechanics, Pennsylvania State University, 227 Hammond Building, University Park, \\ Pennsylvania 16802, USA \\ Hans Irschik, Cochair \\ Division of Technical Mechanics, Johannes Kepler University, Altenbergerstrasse 69, A-4040 Linz, Austria
}

Chair's Introduction-1:55

Invited Papers

2:00

4pSAa1. Zonal and global control of structural intensity in an infinite elastic plate. Sabih I. Hayek, Jungyun Won (Active Vib. Control Lab., Dept. of Eng. Sci. and Mech., Penn State Univ., University Park, PA 16802, hesm@engr.psu.edu), and Giman Kimª) (Kumoh Natl. Univ. of Technol., Kumi, Kyungbuk, 703-701, Korea)

In a previous paper, the active control of active structural intensity (SI) in an infinite elastic plate was discussed. The plate is excited by mechanical noise sources, which generate a vector active SI field in the plate. A colocated point force and a point moment actuator at an arbitrary location on the plate were used. An algorithm was developed to minimize the active SI at a reference point on the plate. The control strategy was aimed at minimization of only the SI at a reference point. In this paper, the active control of SI in a desired zone or a large global area of the plate is explored through the use of multiple controllers spread over the plate. Furthermore, the cost function to be minimized also includes the required power injected by the controllers. Thus the minimization algorithm minimizes both the total SI in a zone as well as the total injected power by the controllers. The influence of the number and type of controllers, and the location of these controllers relative to the source region for the minimization of both the total SI in a region and the input mechanical power of these controllers, is explored. ${ }^{\text {a) }}$ Currently Visiting Scholar at Penn State Univ.

\section{2:20}

4pSAa2. A comparison between active and semi-active global vibration control of structures. Michael J. Brennan and Jedol Dayou (Inst. of Sound and Vib. Res., Univ. of Southampton, Southampton, Hampshire SO17 1BJ, UK, mjb@isvr.soton.ac.uk)

Semi-active devices, such as tunable vibration neutralizers, have been used for a number of years to control structural vibration at the point where the device is attached to the structure. More recently there has been some work on the use of these devices to control the global vibration of a structure and hence the radiated sound. In parallel with this, research has been conducted on the active control of sound radiation from structures. Because there are a number of advantages in using semi-active rather than fully active control in terms of cost and complexity, it is important to be able to quantify the benefits of each strategy on the global control of vibration. The aim of this paper, therefore, is to compare the effectiveness of the two control strategies by focusing on a simple structure such as a beam. Simulations will be presented to show that, provided the dynamic behavior of the structure and the semi-active device are known, then a semi-active device can be appropriately positioned so that it will perform almost as well as the fully active system. Moreover, it will be shown that a simple local control strategy can be employed provided that the device is positioned correctly.

\section{2:40}

4pSAa3. Compact, inexpensive, analog robust controller modules for cabin noise control. Vasundara V. Varadan, Ramanathan Sreenivasan, Woosuk Chang, and Vijay K. Varadan (Penn State Univ., 149 Hammond Bldg., University Park, PA 16802, vvvesm@engr.psu.edu)

Control of noise in an enclosure when the noise source is exterior to the enclosure has applications to rooms, automobiles, helicopters, airplanes, and other vehicles. Reducing the interior noise to acceptable levels is necessary for safety and comfort. One approach to control of interior noise is through vibration suppression of the trim panels that often line the interior of such enclosures. It is ultimately these trim panels that reradiate the noise to the interior. In order to actively control the vibrations of the trim panels, using discrete piezoelectric sensors and actuators, control electronics are needed. Adaptive digital control, while being efficient and powerful, also requires the presence of a computer and DSP boards. There may also be frequency limitations posed by the speed of available DSP boards. An alternative approach is to design compact and inexpensive analog controller units, which also offers the possibility of having such controllers designed as ASIC chips. To demonstrate the efficacy of this approach, results will be presented for the control of noise in a cabin with multiple trim panels when excited by broadband noise from the exterior using MEMS vibration sensors and PZT actuators. 
4pSAa4. Active control of acoustic and structural intensities of a submerged elastic plate. Matthias Fischer and Sabih I. Hayek (Active Vib. Control Lab., Dept. of Eng. Sci. and Mech., 227 Hammond Bldg., Penn State Univ., University Park, PA 16802)

In this paper, the active control of active structural intensity (SI) and the resultant radiated active acoustic intensity (AI) in a coupled structurefluid system is explored. The system consists of an infinite elastic plate submerged in an infinite heavy acoustic medium. The plate is excited to vibration by mechanical noise sources, which generate a vector-active SI field in the plate and a vector AI field in the coupled acoustic medium. Due to heavy fluid loading, the flexural waves in the plate are strongly coupled to the acoustic waves. A line-force and line-moment actuators, which are located at arbitrary locations on the plate, are used for control. The mechanical noise source and control actuators interact not only by their mutual flexural impedance through the elastic plate, they also interact through an acoustic path as well. The control algorithm is aimed at minimizing both SI and AI. The cost function to be minimized consists of a weighted sum of the SI and AI in the far field of the mechanical source region. The influence of the location of these controllers relative to the source region and the relative weighting given to SI versus $\mathrm{AI}$ in the cost function is explored.
4pSAa5. Direct adaptive predictive control using gradient descent. Kenneth Eure, Jer-Nan Juang, and Richard Silcox (NASA Langley Res. Ctr., Hampton, VA 23681, j.juang@LaRC.NASA.Gov)

Predictive control has been successfully used for the regulation of plate vibrations. Past implementations of predictive control have included both feedforward and feedback algorithms. These algorithms may be used as a nonadaptive or an adaptive control method as long as conditional updating is employed or a sufficient level of dither is added to the control signals in order to maintain input and output data coherence for system identification. For large-order systems with many input and outputs, the adaptation rate is severely limited due to the computational burden. The direct adaptive controller presented in this paper uses a gradient descent method to directly determine the predictive control parameters for the feedback regulator. The key features of this control scheme include the use of leakage to prevent drifting of the identified parameters in the presence of closed loop data. The initial information needed by the control scheme is an estimate of the plant order and an estimate of the plant pulse response from input/output data. Because this scheme is adaptive, the controller poses robustness against errors in the estimate of the plant's pulse response which allows the time-varying characteristics of the plant to be regulated.

\title{
Session 4pSAb International Workshop on Active Noise and Vibration Control
}

\section{Structural Acoustics and Vibration: Other Applications of Active Control}

\author{
Nick Doelman, Cochair \\ TNO, Stieltjesweg 1, P.O. Box 155, 2600 AD Delft, The Netherlands \\ Kam Ng, Cochair \\ Office of Naval Research, 800 North Quincy Street, Arlington, Virginia 22217-5000, USA
}

Invited Paper

\begin{abstract}
2:00
4pSAb1. Unsteady dynamics and control of marine thrusters. Ralf Bachmayer and Louis Whitcomb (Dept. of Mech. Eng., Johns Hopkins Univ., 123 Latrobe Hall, 3400 N. Charles St., Baltimore, MD 21218)

This paper reports advances in the unsteady dynamical modeling and control of marine thrusters. First, recent advances in finite-dimensional nonlinear dynamical modeling of marine thrusters are reviewed [Healey et al., IEEE J. Oceanic Eng. 20(4), 354-361 (1995); Bacmayer et al., IEEE/MTS Oceans'98]. Second, a novel nonlinear model-based control algorithm is presented. Third, the performance of the nonlinear model-based control algorithm is evaluated in comparison with conventional and previously reported thrust control algorithms [Whitcomb and Yoerger, IEEE/MTS Oceans'95 2, 1019-1028 (1995)]. [Work supported by NSF and ONR.]
\end{abstract}

\section{Contributed Papers}

\section{2:20}

4pSAb2. Active noise reduction in an ear terminal. Georg E. Ottesen (SINTEF Telecom and Informatics, N-7034 Trondheim, Norway)

An active ear terminal is being designed to obtain both hearing protection and radio communication in adverse environments. Passive damping is sufficient for high frequencies, but low frequencies must be damped by active methods. Even when low-frequency noise is not harmful to the hearing, it heavily increases the effort of communication. Active noise reduction may use the signal from an outer microphone, or from an inner microphone. Active noise reduction obtained by these two microphone positions are simulated and discussed. Analog and digital filters are considered. The time delay budget of the active system is drawn up. Time delays should be minimized to obtain the best performance of the active noise reduction system. The physical system of transducers with their acoustic surroundings contains time delays which cannot be compensated 
by filtering. These time delays can be determined from the transfer functions of the system. The physical design of the ear terminal is performed with a view to reduce these delays. The signal prosessing delay adds to the delay of the physical system. The total delay determines the upper frequency limit of the active noise reduction system.

\section{2:40}

4pSAb3. The Plymouth Prowler, active noise control exhaust system. Part I: Noise reduction. J. Clay Shipps (Tenneco Automotive, Inc., 1025 W. Nursery Rd., Ste. 102, Linthicum, MD 21090)

An active noise control exhaust system or electronic exhaust system was developed for the Plymouth Prowler to demonstrate the ability to reduce backpressure greatly while also significantly reducing the exhaust noise. An electronic exhaust system is typically composed of passive and active elements. One of the major advantages of electronic exhaust systems is that most of the flow restrictions in the passive elements can be removed; this is reponsible for the backpressure reduction. Reduced backpressure typically results in a $3 \%$ to $6 \%$ horsepower gain at the rated engine speed of the vehicle and increases torque over most of the engine speed range. The electronic exhaust system also has the capability of greatly reducing the exhaust noise. The active elements can produce antinoise at very high levels such that, when combined with the unwanted exhaust noise, an overall decrease in the radiated tailpipe noise occurs. This allows the vehicle to meet the legal passby requirements with significant margin while maintaining appropraite sound quality characteristics. This paper will describe the system developed for the Prowler and the resulting performance gains.

3:00

4pSAb4. The Plymouth Prowler, active noise control exhaust system. Part II: Noise shaping. Timothy E. Meeks and J. Clay Shipps (Tenneco Automotive, Inc., 1025 W. Nursery Rd., Ste. 102, Linthicum, MD 21090)

An active noise control exhaust system or electronic exhaust system was developed for the Plymouth Prowler to demonstrate the ability to reduce exhaust backpressure greatly while creating a legal sports car sound for the vehicle. One of the major advantages of electronic exhaust systems is that flow restrictions in the passive elements can be removed, consequently decreasing the total backpressure. This backpressure reduction typically results in a $3 \%$ to $6 \%$ horsepower gain at the rated engine speed of the vehicle and also increases torque at most engine speeds. The electronic exhaust system also has the capability of shaping the exhaust noise. The active elements can produce antinoise at very high levels such that, when combined with the unwanted exhaust noise, there is a significant reduction of selected harmonics. The software which processes the antinoise can be programmed to use engine speed information to determine which harmonics will comprise the resulting waveform. This control varies the vehicle's sound quality characteristics while meeting passby noise requirements. This paper will describe the system developed for the Prowler and the resulting performance gains and sound quality enhancements.

\section{3:20}

4pSAb5. Design considerations for a low-impedance loudspeaker in active noise control exhaust systems. Steven P. Kahn (Tenneco Automotive, Inc., 1025 W. Nursery Rd., Ste. 102, Linthicum, MD 21090)

To meet the needs of automotive original equipment customers, a lowimpedance loudspeaker has been designed for active noise control exhaust systems that reduces system cost, decreases overall weight, eliminates complexity, and improves reliability. The current design practice is to convert the $12-\mathrm{V}$ dc supply of the car battery to $36 \mathrm{~V}$ dc. This supply voltage is used in conjunction with a switching amplifier to drive the noise canceling loudspeaker. By utilizing a low-impedance loudspeaker, the $12-\mathrm{V}$ dc supply from the battery can be fed directly to the amplifier, thereby eliminating the $\mathrm{dc}-\mathrm{dc}$ converter completely. This approach also simplifies the cable harness and reduces the number of connectors. To develop a low-impedance loudspeaker that allows the realization of these advantages, one must consider the following: output level, electrical damping, frequency bandwidth, power handling, and manufacturability. The interaction and trade-offs between these issues must be understood and optimized. Test results between the current configuration and the new approach will also be presented.

\section{3:40-4:00 Break}

4:00

4pSAb6. Contemplations about the numeric transposition of the generalized Kirchhoff integral with the intention of active noise reduction. Mattias Trimpop (Institut fuer Laermschutz, Arnheimer Str. 107, D-40489 Duesseldorf, Germany) and Detlef Krahe (Bergische Universitaet Wuppertal, D-42097 Wuppertal, Germany)

The method of feedback is often used for reduction of noise in defined areas because feedback is able to compensate chances of the transfer function. However, the feedback system cannot work in some special cases, e.g., in single events or impulse noise no preceded signals parts are existing and usable for prediction. Therefore, the feedforward system has to be used in these cases and the prediction is realized by acquisition of the sound wave before it reaches the interesting point of immission. After the presentation of the one-dimensional feedforward system presented in the last year, now the two- and three-dimensional cases and their mathematical treatments will be discussed. For that purpose acoustic wave fields will be analyzed by the generalized Kirchhoff integral and minimized using sound sources in a defined wave field area. Further, the expected problems in the practical application of the numerical solution will be discussed.

\section{4:20}

4pSAb7. Self-adaptive absorber of underwater sound with electrorheological fluid. Dieter Guicking, Patrick Michaelis, and Helge Neumann (Drittes Physikalisches Institut, Universitaet Goettingen, Buergerstr. 42-44, 37073 Goettingen, Germany, guicking@physik3.gwdg.de)

Laboratory experiments have been made on an adaptive-passive absorber of underwater sound with viscous flow in ERF (electrorheological fluid) as absorption mechanism. The absorber model is of the velocity transformation type where a thin fluid layer is confined between an inertial back mass and a piston. Axial vibration of the piston by the incident sound wave causes a strong radial fluid oscillation and thereby enhances the sound absorption. With ERF as fluid, the reflection coefficient depends on the gap width and the field strength. In the present setup the gap width can be varied by a stepper motor. A two-parameter feedback control strategy employing a genetic algorithm has been developed to adjust the gap width and the field strength to minimize the reflectivity as measured by the two-microphone method in an impedance tube. The optimum of $10 \%$ to $20 \%$ reflection is reached within a few generations of adaptation.

\section{4:40}

4pSAb8. Active noise within the generating/pumping groups of a large hydroelectric plant. Leonardo Lecce, Massimo Viscardi, Bruno Maja, Vincenzo Limone, Mario D'Ischia (Dipartimento Progettazione Aeronautica, Università di Napoli "Federico II," Via Claudio, 21, 80125 Napoli, Italy), and Francesco Di Maso (ENEL (National Agency for Elec. Energy), Naples, Italy)

This work is related to a feasibility study for the implementation of an active noise control system to reduce the hydraulic turbomachine's noise in the Presenzano power plant. Previous studies showed that interested turbines are low frequencies noise sources, generating pure tones at 150 and $200 \mathrm{~Hz}$ (third and fourth harmonics of the turbine's BPF). At these frequencies passive noise control systems are ineffective in front of relevant costs and an active approach was so decided to be tested. In accordance with a classical architecture, the preliminary system was composed of four error microphones, four secondary noise sources, and a digital controller implementing an adaptive digital filter. Main performed activities could be identified through four successive steps: (i) analysis of the 
turbomachine's generated primary field levels and space distribution, (ii) secondary sources generated noise field measurement, ten different loudspeakers locations were investigated at this stage; (iii) sensor and actuator locations' optimization by the use of a genetic algorithm's procedure, and (iv) active noise control tests. A mean reduction of $15 \mathrm{~dB}$ at $150 \mathrm{~Hz}$ and $7.5 \mathrm{~dB}$ at $200 \mathrm{~Hz}$ was measured at the error sensors during these tests, revealing the good opportunities of such an approach but also the opportune improvement to pass at a practical implementation.

\section{5:00}

4pSAb9. Sound reflex compensation in active noise control. Zbigniew Ogonowski (Inst. of Automatic Control, Silesian Tech. Univ., ul. Akademicka 16, PL-44-101 Gliwice, Poland, zogonowski@ia.polsl.gliwice.pl)

Creation of a zone of quiet in an enclosure by using active noise control (ANC) methods causes problems with additional noise generated by a reflection of secondary sound. The paper presents a new method of the reflected sound compensation. The idea is to augment the main ANC controller with an auto-feedback controller (AFC). No additional measurement is needed because AFC is fed back with the main controller output. Increase of delay time of the successive reflection assures nonanticipation of the compensation. AFC corrects an activation of the secondary source of sound to cancel the reflected secondary sound generated by the main controller. AFC synthesis uses a mathematical model of the reflection. A specially designed method of the model identification using multisinusoidal excitation is described in the paper. Usually, geometry of the enclosure is constant, thus stationarity of the model is assured. The choice of the model structure implying stability issues is discussed in the paper. Stability of the AFC loop depends also on the reflection delay time. A special stabilizing design method is presented in the paper. An illustrative example of the method application is described.

\section{$5: 20$}

4pSAb10. Active volume sound absorber. Alexander A. Mazanikov (Dept. of Appl. Mathematics, Murmansk State Tech. Univ., MGTU, Sportivnaia St., 13, Murmansk, 183010, Russia, Alexander.Mazanikov@mstu.edu.ru)

The absorbtion cross section (ACS) of a passive volume sound absorber asymptotically equals its geometric cross section. On the other hand, the ACS of an active absorber principally could be arbitrarily large. However, the increase of the ACS results in a rapid growth of the near acoustic fields. This puts restrictions on the practically achievable values of the ACS. The talk presents the results of a simulation of sound field levels generated by active absorbers of spherical shape and different wave sizes in response to incident plane waves. It is shown that near the absorber the level of the sound field increases as compared to the passive absorber of the same size. In the far zone the field of the active absorber corresponds to the field of the passive absorber with larger geometric scale. It is demonstrated that sound energy is absorbed rather on the back side of the absorber then on its front face (with respect to the direction of incident wave).

\title{
Session 4pSCa
}

\section{Speech Communication: Parameters of F0}

\author{
Bernd Möbius, Cochair \\ Lucent Technologies, 600 Mountain Avenue, Murray Hill, New Jersey 07974-0636, USA \\ Thomas Portele, Cochair \\ IKP, University of Bonn, Bonn, Germany
}

Contributed Papers

\begin{abstract}
2:00
4pSCa1. Comparing the naturalness of several approaches for generating $\boldsymbol{F O}$ contours in German text-to-speech systems. Hansjrg Mixdorff and Dieter Mehnert (TU Dresden, Mommsenstr. 13, 01062 Dresden, Germany, mixdorff@teles.de)
\end{abstract}

Generating near-to-natural $F 0$ contours is an important issue in textto-speech synthesis and contributes vastly to the quality of synthetic speech. In earlier studies by the authors, a model of German intonation was developed that is based on the quantitative Fujisaki model. A typical $F 0$ contour is described as a sequence of major rises and falls, which are modeled by onsets and offsets of accent commands connected to accented syllables. The current paper addresses perception experiments comparing the intonational naturalness of a Fujisaki-model-based TTS and four other German TTS systems with comparably high segmental quality. Natural speech samples were used as a reference. Three of the TTS systems had PSOLA, and one LPC segmentals. Two types of experiments were conducted with 20 subjects: (1) a pair comparison of 15 isolated sentences, (2) a ranking test based on a news passage of about $15 \mathrm{sec}$ produced with each of the systems. Preliminary results from experiment (1) show, that on a naturalness scale from 0 to 5 , the natural speech samples reach a maximum score of 4.5, with values of 2.8 for the best synthesis, the LPC-based one. The system with Fujisaki-model-based intonation leads the group of PSOLA systems, which is closely clustered at a mean of 2.4.

\section{2:20}

4pSCa2. Quantifying correlations in pitch- and amplitude contours. Hans-Peter Herzel (Inst. Theor. Biol., Humboldt Univ., Invalidenstr. 43, D-10115 Berlin, Germany, h.herzel@biologie.hu-berlin.de) and Robert Reuter (Inst. Electron. Techn. Univ., Berlin, Germany)

Nonlinear phenomena such as subharmonics, biphonation, or deterministic chaos induce characteristic patterns in pitch and amplitude contours. A novel measure of correlations in contours termed MAC (mean average correlation) is introduced. For uncorrelated and weakly correlated noise, the expectation values and standard deviations of MAC are calculated. It is shown that trend elimination induces spurious correlations. Moreover, simulations of the circle map are analyzed and high values of MAC for subharmonic and chaotic data are found. Examples are presented of severely rough voices and excised larynx data and correlations are 
quantified in the corresponding contours by MAC. Finally, the connection between perceived voice quality and MAC is studied with the aid of 120 perceptually evaluated voices. [Work supported by the Deutsche Forschungsgemeinschaft.]

\section{2:40}

4pSCa3. Influences of various factors upon parameters of the command-response model for fundamental frequency contour generation. Sumio Ohno, Yoshikazu Hara, and Hiroya Fujisaki (Dept. of Appl. Electron., Sci. Univ. of Tokyo, 2641 Yamazaki, Noda, 278-8510 Japan, ohno@te.noda.sut.ac.jp)

The command-response model by Fujisaki and his co-workers formulates the generation process of the fundamental frequency contour (henceforth $F 0$ contour) in terms of a set of input commands carrying linguistic and paralinguistic information and the mechanisms that respond to these commands. The parameters of the mechanisms are time constant of the phrase control mechanism $(\alpha)$, that of the accent control mechanism $(\beta)$, and the baseline frequency $(F b)$. The present study investigates the influences of speech rate, speaking style, and individual difference on these parameters as well as their variability. The speech material consisted of recordings of a short story by six native speakers of Japanese at three speech rates (slow, normal, and fast) and in two speaking styles (reading and conversational). The parameters were extracted by the method of analysis-by-synthesis, and the results were analyzed statistically. The analysis indicated that utterance-to-utterance variations are quite small in all three parameters for a given rate, style, and speaker. Among the three parameters, only $\alpha$ showed a small but systematic tendency to increase with the speech rate, while differences in speaking style did not affect these parameters. Individual differences were quite small in $\alpha$ and $\beta$, while $F b$ varied from speaker to speaker.

\section{3:00}

4pSCa4. Application of the command-response model to the analysis, interpretation, and synthesis of fundamental frequency contours of speech of various languages. Hiroya Fujisaki and Sumio Ohno (Dept. of Appl. Electron., Sci. Univ. of Tokyo, 2641 Yamazaki, Noda, 278-8510 Japan, fujisaki@te.noda.sut.ac.jp)

A command-response model has been presented by Fujisaki and his co-workers initially for the process of generation of the fundamental frequency contour (henceforth $F 0$ contour) of the common Japanese. It consists of a set of input commands carrying linguistic and paralinguistic information, and the mechanisms that respond to these commands to generate both phrase and accent components, which, together with a baseline value, constitute the actual $F 0$ contour. Subsequent works have shown that the model is applicable also to $F 0$ contours of some other dialects of Japanese as well as of other languages including Chinese, English, German, Greek, Spanish, and Swedish. It has been argued, however, that the model may not be able to generate certain contour types that are not found in Japanese but are commonly used in other languages [e.g., P. Tayler, Speech Commun. 15, 183]. This paper shows how these contour types can actually be generated by the same mechanisms, with certain languagespecific timing, polarity and magnitude of the input commands, all within the framework of syntactic and prosodic constraints of each language. The results will be demonstrated by resynthesized speech, and testify the validity and usefulness of the model for multilingual text-to-speech synthesis.

\section{3:20}

4pSCa5. Iconic prosody and information in spontaneous oral narrative. Guillermo Andrés Toledo (CIRAL, Fac. Lettres, Univ. Laval, Québec, QC G1K 7P4, Canada and LIS-CONICET, Argentina)

The information interchange on cognitive categories of the mental representation of referents (concepts) interrelated with prosodic marking (levels of phonological prominence) have been studied. Speakers actualize a limited amount of information inactivating backwards. These cognitive processes appear in three states of the referents: activation, semi- activation, and inactivation. In activation, a concept would be focalized in actual stream of consciousness. In semi-activation, the information is situated in a peripheral zone of consciousness: the concept was already activated. In inactivation, the concept would be activated in the hearer's conscience for the first time. These states were analyzed through data extracted from spontaneous narrative discourses obtained from an Argentinean Spanish speaker. The iconicity of prosodic prominences, a correlation between the phonetic encoding and the mental status of information weight, was explored acoustically. Measurements were made through $F 0$ data normalized by a logarithmic $z$-score transformation calculated in the highest value of an item classified among one of the three states. Findings were similar to previous research on semi-spontaneous materials [G. Toledo, 16th International Congress on Acoustics, 4aSC1]: lower prominences were the marking of activation and semi-activation, and higher prominences were the feature of inactivation, i.e., a degree of prosodic iconicity.

3:40-4:00 Break

4:00

4pSCa6. A phonological interpretation of the "Gussenhoven-Rietveld Effect." Stefanie Jannedy and Mary E. Beckman (Dept. of Linguist., Ohio State Univ., Columbus, OH 43210)

Dutch listeners rate the perceived prominence of the second fundamental frequency $(F 0)$ peak $(\mathrm{P} 2)$ to increase as the first peak $(\mathrm{P} 1)$ increases and decreases in $F 0$ while $\mathrm{P} 2$ is held constant. This GussenhovenRietveld effect was replicated with English listeners [Ladd et al. (1994)]. They interpret the positive correlation between the frequency of P1 and the perceived prominence of P2 as a function of the pitch range. Gussenhoven and Rietveld (1997) attribute this effect to parsing P1 and P2 as occurring in a single phrase, signaled by the intonation contour between the peaks. It is proposed that listeners parse the intonation phrase internal $F 0$ peaks as relational to each other and as good or bad instances of phonological accent categories: An $F 0$ contour with a lower P1 and a higher $\mathrm{P} 2$ can be interpreted as an $H^{*} L+H^{*}$ sequence, with the realization of the $L+H^{*}$ as not prominent for this category. A flat-shaped $F 0$ contour with $\mathrm{P} 1$ and $\mathrm{P} 2$ roughly equal can be interpreted as an $H^{*}$ accent on both peaks. A higher P1 interpolating onto a lower P2 can be interpreted as a rather prominent instance of a downstepped $H^{*}$ accent on P2. This explanation assumes a phonological interpretation of intonation contours.

\section{4:20}

4pSCa7. Changes of voice parameters and melody patterns during the first year of life in human twins. Kathleen Wermke (Inst. of Anthropology, Humboldt Univ. Berlin, Medical Faculty Charite, Tucholskystr. 2, 10117 Berlin, Germany, kathleen.wermke@charite.de), Werner Mende (Berlin-Brandenburg Acad. of Sci., Berlin, Germany), Hanno Borschberg, and Roland Ruppert (Maternity Hospital, Chemnitz, Germany)

The study analyzed changes of cry characteristics of twins using spectrograms, fundamental frequency contours (melody), and several indicators of microvariability of the fundamental frequency $(F 0)$ calculated with the aid of a KAY Elemetrics CSL Model 4305. Subjects were 15 identical and 15 fraternal healthy twin pairs, whose spontaneous utterances were recorded every 6 weeks over the first year of life. Developmental changes of $F 0$, jitter, perturbation quotient, degree of voiceless, degree of subharmonic components as well as the evolution of complex melody patterns were analyzed. Correlations of the voice data with anthropometric data (weight, length, head circumference) and obstetrical factors (time of gestation, presentation, birth modus) were calculated to consider influences by growth discordance or different birth histories. As identical twins have a much greater congenital synchronization of development, universals of prespeech evolution are more convincingly expressed in these pairs (lower intrapair differences). A statistically significant higher intrapair concordance in identical twins was found for the mean $F 0$ within the first week 
of life, for the mean $F 0$ and jitter in the period within 5-8 months of life, and for certain melody patterns. For other parameters, significant intrapair differences between identical and fraternal twins were not found.

\section{4:40}

4pSCa8. On the waveforms and spectra of glottal flow models. Boris Doval and Christophe R. d'Alessandro (LIMSI-CNRS, BP133, F91403 Orsay, France, doval@limsi.fr)

Several glottal flow models have been proposed for speech analysis and synthesis (e.g., LF, Rosenberg, $\mathrm{R}++$, and Klatt). All these models do not use the same number of parameters, or the same name for similar parameters, and it appears difficult to compare their merits. Then, a unified framework for studying the time and frequency domain properties of glottal flow models is proposed. It is shown that all the models can be represented by a common set of five time-domain parameters: three scale parameters ( $T 0$, peak amplitude, open quotient), a shape parameter (asymmetry quotient), and a closure continuity parameter. A generating function is computed for each model by normalization of the model with respect to scale parameters and closure continuity parameter. The specific features of each model are represented in its generating function. The spectrum of generating functions is low pass, and its derivative can be characterized by a spectral maximum, coined "glottal formant." The closure continuity parameter corresponds to a spectral tilt component. The scale parameters are interpreted using scaling properties of the Fourier transform. Then, the glottal flow spectra can be characterized by two breakpoints. Frequencies of these breakpoints can be computed analytically for each model parameter setting.

\section{5:00}

4pSCa9. Analysis of pitch accent categories in Welsh. Nicholas J. Kibre (Univ. of California, Santa Barbara, CA)

This paper describes the results of a study of pitch accent categories in Welsh, and their distribution in natural speech. In addition to these descriptive findings, it describes the development of a data-driven approach to intonation. To be useful for the analysis of natural speech, an intonational model must provide means for determining an unambiguous phonological representation for any observed speech token. One approach which will be addressed is the Tilt Model [K. Dusterhoff and A. Black, ESCA Workshop on Intonation (1997); P. Taylor, ICSLP (1998)], amended as necessary to account for the accent patterns of Welsh. This will be compared with an alternative of using statistical methods, particularly factor analysis, to determine the underlying descriptive dimensions of pitch accents in the language. The resulting model will be applied to a corpus of spoken Welsh utterances, and initial findings on the distribution of accent types will be reported.
4pSCa10. Empirical eigenfunctions obtained from high-speed imaging of the vocal folds. David A. Berry, Ingo R. Titze (Dept. of Speech Pathol. and Audiol., Univ. of Iowa, Iowa City, IA 52242), and Hanspeter Herzel (Humbold Univ., Berlin, Germany)

Previously, the method of empirical eigenfunctions has been used to interpret both periodic and aperiodic oscillations from a finite-element model of vocal fold vibration. For a typical case of periodic vibrations from the model, it was shown that two eigenfunctions explained $98 \%$ of the variance of the nodal trajectories. Furthermore, the extracted eigenfunctions were qualitatively similar to analytic normal modes computed from a linear continuum model. Even for complex aperiodic oscillations, $85 \%$ of the variance could be explained by just three eigenfunctions. The method of empirical eigenfunctions is now applied to quantitative observations of vocal fold movement obtained from excised canine larynx experiments. With the aid of a high-speed imaging system (the Kodak EktaPro 4540), both periodic and aperiodic oscillations were captured. The newly calculated eigenfunctions will be presented, and contrasted with those obtained previously from finite element simulations. Hints of low dimensionality and mechanisms of irregular oscillation will be discussed. [Work supported by NIH, Grant No. R29 DC03072.]

\section{$5: 40$}

4pSCa11. A design of laryngeal structures for a physiological articulatory model. Chao-Min Wu (ATR Human Information Processing Res. Labs., 2-2 Hikaridai, Seika-cho, Soraku-gun, Kyoto, 619-02 Japan), Jianwu Dang (ATR Human Information Processing Res. Labs., Kyoto, 619-02 Japan and Univ. of Waterloo, Waterloo, ON N2L 3G1, Canada), and Kiyoshi Honda (ATR Human Information Processing Res. Labs., Kyoto, 619-02 Japan)

A model of the hyoid-larynx complex is designed and incorporated into a physiological articulatory model. The previously reported articulatory model [Dang and Honda, Proc. ICSLP98] consists of the tongue, mandible, hyoid bone, and vocal tract wall. The aim of this work is to include the laryngeal structures to simulate the tongue-larynx interaction which is observed in natural speech. The framework of laryngeal structures is composed of the thyroid, cricoid, and arytenoid cartilages, which are connected by muscles, ligaments, and joints. The form and geometry of the cartilages are extracted from volumetric MR images of a male speaker and represented in a quasi-3D shape. They are modeled to yield joint rotation and translation as well as vertical movements. The ligaments and muscles are represented by mass points with viscoelastic springs, and the cartilages are composed by springs with an extremely large stiffness. Laryngeal factors of F0 control such as cricothyroid rotation and laryngeal vertical movement are demonstrated in the simulation, and plausible dynamic behaviors of the tongue-larynx interaction are observed. The performance of the model is tested by comparison of acoustic output from the model with recorded speech. 


\title{
Session $4 p S C b$
}

\section{Speech Communication and Psychological and Physiological Acoustics: Models of Speech Processing in the Auditory Pathway}

\author{
Steven Greenberg, Cochair \\ International Computer Science Institute, 1947 Center Street, Berkeley, California 94704, USA \\ Roy D. Patterson, Cochair \\ Centre for the Neural Basis of Hearing, Physiology Department, Cambridge University, Cambridge CB2 3EG, UK \\ Chair's Introduction-1:55 \\ Invited Papers
}

2:00

4pSCb1. Time-interval information in the auditory representation of speech sounds. Roy D. Patterson (Ctr. for the Neural Basis of Hearing, Physiol. Dept., Cambridge Univ., Cambridge CB2 3EG, UK)

In the auditory system, the primary fibers that encode mechanical motion of the basilar partition are phase-locked to that motion, and this information is preserved, to varying degrees, up to the inferior colliculus. It is known that this timing-interval information is used in localization, and it is probably also used to separate sources from diffuse background noise. The time intervals are on the order of milliseconds, and so traditional speech preprocessors (like MFCC systems) with frames on the order of $15 \mathrm{~ms}$, remove the time-interval information from the representation. The performance of these systems deteriorates badly when the speaker is in a noisy environment. This suggests that time-interval processing will eventually need to be integrated into speech recognition systems if they are to achieve the kind of noise resistance characteristic of human speech recognition. An auditory image model (AIM) will be presented that is designed to stabilize repeating time-interval patterns like those produced by voiced speech, and results from experiments where AIM has been used as a preprocessor for automatic speech recognition. [Work supported by UK MRC (G9703469).]

\section{2:20}

4pSCb2. On temporal smoothing and speech perception. Oded Ghitza (Bell Labs., Lucent Technologies, 700 Mountain Ave., Murray Hill, NJ 07974)

Neurophysiological and psychophysical studies provide evidence for the existence of temporal integration mechanisms in the auditory system. These may be viewed as low-pass filters, parametrized by their cutoff frequencies. It is of interest to specify these cutoffs, particularly for tasks germane to the effect of temporal smoothing on speech quality, within the context of speech-coding strategies (e.g., what is the smallest number of bits with which to represent temporally smoothed speech that is perceptually indistinguishable from the original?). In order to answer such questions, speech-processing rules have been derived so as to create synthetic speech capable of generating model auditory-nerve (AN) firing patterns temporally smoothed in a manner commensurate with that observed in neurophysiological studies of the AN (e.g., at low characteristic frequencies, neural discharges of AN fibers are phase locked to the underlying driving critical-band signal, i.e., synchrony is maintained; at high CFs, temporal information is preserved by the instantaneous average rate of the neural firing pattern, which is related to the temporal envelope of the underlying driving signal). This presentation describes the processing rules and the results of psychophysical experiments performed to measure the cutoff frequencies of these filters within the context of speech quality.

4pSCb3. Modeling speech intelligibility and quality on the basis of the "effective" signal processing in the auditory system. Birger Kollmeier, Matthias Wesselkamp, ${ }^{\text {a) }}$ Martin Hansen, ${ }^{\text {b) }}$ and Torsten Dau (AG Medizinische Physik, Universität Oldenburg, Germany)

A model of the "effective" signal processing performed by the auditory system is applied to the quantitative assessment of speech intelligibility (cf., Kollmeier et al., Proc. ICA 95, III, 81-84). It consists of a gamma one filterbank followed by envelope extraction, nonlinear adaptation circuits, and subsequent modulation low-pass filtering in each filter channel before being fed into a crosscorrelation-type detector or pattern recognizer. The model is capable of predicting the speech intelligibility of both normal and hearing-impaired listeners under a wide range of signal-to-noise ratios, as well as the perceived quality of speech signals whose spectro-temporal properties are systematically degraded. The degradations are generated with a modulated noise reference unit either in a band-specific, time-independent way or in a broadband, temporally varying way. These intelligibility and quality assessment data can be used to deduce the components of the model most germane to the processing of speech, as well as provide information about how to enhance and extend the model for better predictive performance. ${ }^{a)}$ Now at Siemens Audiologische Technik, Erlangen, Germany. b)Now at Widex Hearing Aids, Vaerloese, Denmark. 
4pSCb4. Periodotopic organization in the ICC of gerbils: A 2-deoxyglucose study. Gerald Langner and S. Braun (Neuroacoust., Zoological Inst., Tech. Univ. of Darmstadt, Schnittspahnstr. 3, D-64287, Darmstadt, Germany)

Periodicity is an important property of many acoustic signals. In the central inferior colliculus (ICC) of the auditory brainstem pathway, frequency and periodicity information converge on a tonotopically organized network. Electrophysiological investigations demonstrate a marked degradation of temporal information in the ICC pertaining to the envelope of spectrally complex signals relative to that observed at the level of the auditory periphery. The temporal information appears to be transformed into a rate-place code and is represented topographically in an orientation orthogonal to the spectral frequency (tonotopic) axis. Additional evidence for orthogonal orientation of frequency and periodicity is derived from a magnetoencephalographic (MEG) study of human auditory cortex and from optical recordings in cat auditory cortex. 2-deoxyglucose labeling was used to investigate the topographic organization of frequency and periodicity information in the ICC of the Mongolian gerbil. The results of this study provide additional evidence for such topographic orthogonality, as well as for convergent information flow, suggesting that neurons in the ICC project to a specific subpopulation of cells in the low-frequency area of the auditory cortex that integrate information across separate periodotopically organized frequency bands.

\section{3:20-3:40 Discussion}

\section{3:40-4:00 Break}

4:00

4pSCb5. The representation of spectrally dynamic signals in primary auditory cortex. Shihab Shamma (Dept. of Elec. Eng., Univ. of Maryland, College Park, MD 20742)

Natural sounds, including speech, are traditionally characterized by their patterns of pitch, timbre, loudness, and modulation, all of which are closely related to the instantaneous spectrum of these signals. The central auditory system has developed elegant mechanisms to encode this spectro-temporal information. Thus, at the level of the primary auditory cortex (AI), the dynamic spectrum is repeatedly represented over a wide range of spectral and temporal resolutions. This multi-scale representation is derived from response patterns of cells selective to such parameters as local bandwidth, asymmetry of spectral peaks, and onset/offset transition rates. Physiological experiments measured the spectral and dynamic properties of neurons in AI using linear system analysis. One set of experiments used broadband signals with sinusoidally modulated spectral envelopes ("ripples"). Varying the density (or frequency), amplitude, phase, and velocity of the ripple pattern in a systematic fashion was used to derive a ripple transfer function. From this function it is possible to derive a spectro-temporal (impulse) response function via an inverse Fourier transform. A separate technique computed the neuronal impulse response (via reverse correlation) using signals with random and dynamic spectral characteristics. This presentation summarizes the application of these two analytical techniques to characterizing AI neuronal responses and discusses the scientific and technical implications of these multi-scale, cortical representations.

\section{4:20}

4pSCb6. Representation of consonant-vowel syllables in mammalian auditory cortex: Effects of background noise and speaking rate. Christoph E. Schreiner, Sarah Wong, Ben Bonham, Steve Cheung, and Purvis Bedenbaugh (Coleman Lab., W. M. Keck Ctr. for Integrative Neurosci. and Sloan Ctr. for Theoretical Neurobiology Univ. of California, San Francisco, CA 94143-0732)

The representation of synthetic and naturally spoken consonant-vowel (CV) syllables was investigated in the auditory cortex of the cat and the squirrel monkey. Spatially discrete and temporally synchronized patterns of neuronal activation are systematically distributed across the tonotopic axis. This activation pattern is largely determined by the dynamics of the signal's spectro-temporal envelope (such as the energy modulation associated with plosive consonants and the onset of voicing). The spatio-temporal activity is strongly influenced by sound pressure level, resulting in significant changes in neuronal response strength and distribution (analogous to those associated with sinusoidal signals). In contrast, background noise has a much smaller impact on the excitation pattern, except at levels sufficiently high as to cause extensive suppression of overall activity. Varying the speaking rate (i.e., shortening vocalic durations and the interval separating CV syllables) was systematically investigated. Forward masking and adaptation affect the magnitude, latency, and spatial distribution of neuronal activity for both slow and rapid speaking rates. These effects are differentially distributed across cortical fields. [Work supported by grants from NINDS and NSF.]

\section{4:40}

4pSCb7. Magnetoencephalographic evidence for temporal representations in human auditory cortex. David E. Poeppel (Dept. of Linguist., Univ. of Maryland, 1401 Marie Mount Hall, College Park, MD 20742), Timmothy P. L. Roberts (Univ. of California, San Francisco, CA 94143), and Steven Greenberg (Intl. Computer Sci. Inst., Berkeley, CA 94704)

It is generally assumed that the auditory representation of speech is derived primarily from the spatial distribution of activity across the tonotopically organized neuronal axis. Such temporal parameters as spike synchronization, interspike interval, and neuronal oscillations are also regarded as potential complements to the classical tonotopic representation of the speech spectrum. The present study used magnetoencephalography (MEG) to investigate the temporal behavior of large neuronal ensembles in human auditory cortex. Experimental stimuli were sinusoidal and square-wave signals, sinusoidally amplitude-modulated tones, and synthetic vowels. The results indicate the presence of a systematic representation of the signal spectrum and pitch derived from the latency pattern of the major auditory-evoked neuromagnetic field. The magnitude of latency differential to signals of variable frequency and pitch can be as large as $25 \mathrm{~ms}$. This "tono-chronic" analysis may offer an alternative encoding mechanism for pitch and spectral information that plays an important role in the perception of complex signals such as speech. [Research supported by the James S. McDonnell Foundation and the National Science Foundation.] 
4pSCb8. An oscillatory correlation framework for the separation of speech from interfering sounds. Guy J. Brown (Dept. of Comput. Sci., Univ. of Sheffield, Regent Court, 211 Portobello St., Sheffield S1 4DP, UK, g.brown@dcs.shef.ac.uk) and DeLiang L. Wang (The Ohio State Univ., Columbus, OH 43210-1277)

Speech is seldom heard in isolation-usually, a mixture of sounds reaches our ears. Hence, the auditory system is faced with a scene analysis problem-it must group together spectral components that are likely to have arisen from the same environmental source. At the neurobiological level the auditory system also faces a binding problem-it must construct meaningful wholes from features that are represented in separate neural structures. Models of auditory function based on the theory of oscillatory correlation offer a potential solution to the scene analysis and binding problems. According to this theory, perceptual streams are represented by populations of neural oscillators, such that oscillators belonging to the same stream are synchronized (phase locked with zero phase lag), and are desynchronized from oscillators belonging to different streams. A neural oscillator model of auditory function has been developed, capable of segregating speech from interfering sound sources. The core of the model is a two-layer neural oscillator network, in which lateral connections among oscillators encode harmonicity as well as proximity in time and frequency. A variant of this model is able to account for the sharing of acoustic evidence among streams, as would occur in "duplex" perception of speech.

\title{
$5: 20$
}

4pSCb9. Implications of a sensory-motor theory for the representation and segregation of speech. Neil Todd (Dept. of Psychol., Oxford Rd., Manchester Univ., Manchester M13 9PL, UK)

Discussed is a computational model which attempts to simulate the principal brain structures involved in sensory-motor sequencing, including auditory cortex, posterior parietal cortex, cerebellum, motor cortex, and the motor output system. A particular focus in this presentation is on the auditory cortical component responsible for computing a 3-D spatio-temporal power spectrum of the auditory image flow [N. Todd, Proc. 16th ICA (1998)], which captures information topographically on three levels, roughly corresponding to timbre, pitch, and rhythm, and reflects the temporal resolution of the cochlea, inferior colliculus, and auditory cortex, respectively. By considering the spatio-temporal orientations of cortical receptive fields (RFs), it is possible to delineate a set of stationary and moving primitive features upon which the higher-order constructs of pitch and timbre are built. A separate model population of secondary auditory cortical neurons, which receive inputs from the population of primary RFs, provides a potential means of accounting for a number of important phonological and melodic processes. Implications of this model are discussed for the development of new algorithms for the representation and segregation of speech via self-organization of primary and secondary RFs by means of the principle of information maximization.

5:40-6:00 Discussion

Session 4pSP

\section{Signal Processing in Acoustics: Acoustic Signal Processing for Systems with 2D/3D Sensor Arrays II}

\author{
Stergios Stergiopoulos, Cochair \\ Defence and Civil Institute of Environmental Medicine, P.O. Box 2000, North York, Ontario M3M 3B9, Canada \\ Dieter Kraus, Cochair \\ STN ATLAS Elecktronik GmbH, Naval Division, System Analysis and Signal Processing Group, MME6, \\ Sebaldsbrücker Heestrasse 235, 28305 Bremen, Germany
}

Chair's Introduction-1:55

Invited Papers

2:00

4pSP1. Robust matched-field processing in underwater acoustics. Newell O. Booth (Space and Naval Warfare Systems Ctr., Code D881, San Diego, CA 92152-5000)

During the last decade, underwater acoustic matched-field processing (MFP) has been shown to be a robust and powerful means of extracting information related to the propagation of sound and the location of acoustic sources in the ocean. Using large arrays of acoustic receivers, broadband sources of acoustic energy, and physically accurate propagation models, MFP techniques have been used to detect, localize, and track acoustic sources. It is also possible to estimate the array shape as well as oceanographic parameters which affect acoustic propagation. Using a broad definition of matched-field processing, this paper reviews its application to underwater acoustics and acoustical oceanography. Physical phenomena which contribute to the successful application of MFP are discussed, along with other phenomena which have contributed great simplifications. 
4pSP2. Phase conjugation and matched-field processing. W. A. Kuperman, W. S. Hodgkiss, H. C. Song, Aaron Thode (Marine Physical Lab., Scripps Inst. of Oceanogr., La Jolla, CA 92093-0701), T. Akal, and C. Ferla (SACLANT Undersea Res. Ctr., La Spezia, Italy)

Matched-field processing (MFP) can be implemented with a backpropagation algorithm. The method requires the same knowledge of the environment as the conventional MFP replica search approach. Rather than use numerical backpropagation, the phase conjugation (PC) approach (also referred to as a time-reversal mirror) uses physical sources at each receiver position to backpropagate the phase-conjugated data on the receive array. The acoustic field then focuses at the position of the original radiating source that is the same position of the main lobe of the analogous MFP ambiguity surface. No a priori environmental information is required. Two experiments implementing PC in the ocean have been successfully conducted in 1996 and 1997. These experiments and the physics of PC provide new insight into MFP.

4pSP3. Optimized image processing for multi-beam/multi-aspect sidescan sonar application. Ursula Hoelscher-Hoebing and Dieter Kraus (STN ATLAS Elektronik GmbH, Sebaldsbruecker Heerstrasse 235, D-28305 Bremen, Germany, hoelscher.u@stn-atlas.de)

In the development of future sonor systems, computer-aided classification (CAC) becomes increasingly important. Adequate processing of the multidimensional image data is one important component in a multi-beam/multi-aspect sidescan sonar CAC system. Because the target strength of an object varies with aspect angle, the sonar echo and its representation in a sidescan image is rather random. To make sure that a maximum echo strength is gained, in multi-beam/multi-aspect sidescan sonars overlapping bottom areas are insonified by temporal successive pings, giving the echoes of the target under different aspect angles. Taking advantage of the multi-aspect echo structure in the images of successive pings, they are fused to one sidescan image. Existing image fusion algorithms now require an operator to set threshold values distinguishing target and shadow zones from bottom reverberation zones. An unsupervised method is proposed here for segmentation and optimal fusion of multidimensional images. Its basic steps are image segmentation by means of the EM algorithm; clustering of image segments applying the Markov random field approach; and fusion of successive pings images following a minimum/mean/maximum strategy according to the results of image segmentation and clustering.

3:00

4pSP4. Signal processing algorithm for sound source localization with discretionarily distributed multiple receiving sensors. Nai-chyuan Yen (CLY Assoc., 2109 Rampart Dr., Alexandria, VA 22309-1537, CLY-yen@juno.com)

The deployment of strategically placed multiple receiving sensors has many practical ways to detect, localize, and classify a sound source in situations such as monitoring an isolated street disturbance in an urban environment with posted microphones or identifying a submerged object in the ocean with air-dropped sonobuoys. A simple processsing algorithm based on signals received from multiple sensors is derived from the information contained in the cross-spectrum matrix formed from the receiving sensors. Using the magnitude distribution of eigenvalues across the frequency bandwidth as the indication for the strength of signal excess in the frequency domain, their corresponding eigenvectors provide the required complex number weightings to select the related coherence components of the signal. By examining the phase angle data from each sensor's weighting factor, a set of simultaneous algebra equations can be formulated to estimate the location of the sound source. The computation procedure of this approach is uncomplicated in comparison with the traditional triangulation method. Furthermore, the additional accompanied computed eigenvalues can be utilized for the characterization of the detected sound source, thereby establishing its identification reference. Simulated examples are used to test out the effectiveness of this multiple sensor sound source evaluation methodology at various operational scenarios.

4pSP5. Optimal amplitude shading for arrays of irregularly spaced or non-coplanar elements. Timothy C. Gallaudet and Christian P. de Moustier (Marine Physical Lab., Scripps Inst. of Oceanogr., 9500 Gilman Dr., La Jolla, CA 92093-0205)

The majority of optimal amplitude shading methods for arrays of irregularly spaced or non-coplanar elements rely on numerical optimizations and iterative techniques to compute the desired weighting function because analytic solutions generally do not exist. Optimality is meant here in the Dolph-Chebyshev sense to provide the narrowest mainlobe width for a given sidelobe level. A simpler and more efficient technique to compute the shading weights for arbitrary line array shapes or element spacings is presented and it is shown that it is sufficient to sample the optimal Dolph-Chebyshev window, computed for a uniform line array of equivalent aperture length, at the element position of the nonuniform array. Examples are given for narrow-band plane-wave beamforming with curved arrays in which phase compensation is achieved by projecting the elements on a line tangent to the array. For the same mainlobe width, the resulting peak sidelobe levels are within $3 \mathrm{~dB}$ of a 30-dB Dolph-Chebyshev weighted uniform line array of equal aperture length and number of elements. Results are presented for computer simulations and for data collected at sea with the Toroidal Volume Search Sonar by the Coastal Systems Station, Panama City, Florida. [Work sponsored by ONR-NRL (Contract No. N00014-96-1G913).] 
3:40

4pSP6. Localization of the sources using blind identification of signal subspace. Bendjama Ammar and Bourennane Salah (S. D. E. M., URA, CNRS 2053, B. P. 52, Quartier Grossetti, 20250 Corte, France)

To find the direction of arrival (DOA) of $N$ sources impinging on an array of $M$ sensors, actual fourth-order direction-finding methods try to solve an $\mathrm{N}$-dimensional problem from the statistics of the data. Current narrow-band array processing techniques are based on the second-order statistics of the received signals. In many situations, the received signals are non-Gaussian, so that they contain valuable statistical information in their moments of order greater than two. In these circumstances, it makes sense to develop array processing techniques which are designed to use their higher-order information. Of particular interest are the algorithms based on higher-order cumulants, which are asymptotically insensitive to Gaussian noise: it is thus neither necessary to know, to model, or to estimate the noise covariance, which is a reasonable assumption in many practical situations. In this paper, an approach of direction finding is proposed, based on the first step of blind identification of source steering. As a second step, the direction-finding concept is now possible due to the development, these last years, of several blind source separation methods which allow the blind identification of source-steering vectors.

\section{4:00-4:20 Break}

\section{4:20}

4pSP7. Blind source separation by convex optimization to resolution enhancement. Kenbu Teramoto and Noriko Mori (Dept. of Information Sci., Saga Univ., Saga, 8408502 Japan)

In the current blind source separation (BSS) one tries to separate statistically independent unknown source signals from their linear mixtures without explicit knowledge of the mixing coefficients. However, in the case that the sources radiate statistically dependent signals, difficulties exist in separating sources. In such cases, it is important to utilize the nonlinear convex constraints. In this paper, a convex optimization method for BSS and parameter estimation of unknown components in the signal transfer function is proposed. This technique relies upon seeking the unique KKT (Karush-Kuhn-Tucker) point of the augmented Lagrange function which is defined over the direct product of source object space and observed signal space. Utilizing prior knowledge about spatial characteristics of the point spread function and short-time statistical characteristics of noise concurrently, the proposed algorithm can converge to the reliable solution on the contrary to the independent component analysis (ICA) exhibiting oscillations. Applying the proposed method to a 3-D sparse aperture holographic sonar which has a limited number of transducers distributed sparsely, the novel method yields features that reduce the artifacts caused by under-sampled data and achieve higher resolution with higher convergence rate to the optimal solution than that of ICA.

\section{4:40}

4pSP8. Forward/backward first-order statistics algorithm for the estimation of DOA in a multipath environment. Joon-il Song, Han-Su Kim, Koeng-Mo Sung (Appl. Acoust. Lab, Inst. of New Media and Commun., Seoul Natl. Univ., Kwanak-Ku, Seoul 151-742, Korea), and Jun-Seok Lim (Sejong Univ.)

Many kinds of direction of arrival (DOA) estimation methods in the coherent environments have been studied for many decades. Most of them use spatial smoothing to tackle the coherent environments. An alternative method was proposed by Pillai which used first-order statistics on each sensor datum [S. U. Pillai, Array Signal Processing (Springer-Verlag, New York, 1989)]. The method handled the complex numbered input data with additional symmetric array so that it needed two times as many sensors as it needed in the case of the real numbered input data. In this paper a DOA estimation method using forward/backward first-order statistics is proposed which can handle the complex numbered input data without additional sensors. [Work supported by UARC.]

\section{5:00}

4pSP9. Robust Kalman-based DOA estimator with variable fading memory. Han-Su Kim, Koeng-Mo Sung (School of Elec. Eng., Seoul Natl. Univ., Seoul, Korea), and Jun-Seok Lim (Sejong Univ.)

In this paper, VFM (variable fading memory) is introduced to the robust Kalman-based DOA (direction of arrival) estimator [Han-Su Kim and Koeng-Mo Sung, Proc. 16th ICA/135th ASA 1, 1327-1328 (1998)] for DOA estimation of uncorrelated narrow-band plane waves under the impulsive noise environment. To introduce VFM properly into the Kalman filter, the proposed algorithm is derived from a modified least-squares criterion with VFM for DOA estimation. Since the derived VFM can be adaptively calculated with respect to the estimation error, the proposed algorithm has the effectiveness to the impulsive noise in stationary or nonstationary situations. The introduction of VFM enables the timevarying DOA to be estimated properly and prevents the estimated error caused by the impulsive outlier from propagating into future time instants, adaptively decreasing the importance of past observations. The proposed method can make the small perturbation to the parameters using the Huber function, named $M$-estimation, and adaptively reduce the estimation error propagation caused by past observations of the impulsive noise using VFF. Compared to the conventional Kalman algorithms, simulations show that the proposed algorithm is robuster than any other conventional Kalman algorithms to the impulsive noise.

\section{$5: 20$}

4pSP10. Frequency-angle selectivity under conditions of 3-D antenna motion. Igor I. Gorban and Sergey P. Volkovetsky (Inst. of Math. Machines and Systems, 42 Ave. Acad. Glushkov, Kiev, Ukraine, 252187, gorban@immsp.kiev.ua)

Common estimation of frequency-angle signal parameters under conditions of complicated 3-D antenna motions with angle rotations around the space axes is researched. A program package for modeling has been developed. The package calculates Fisher informative matrix and CramerRao estimates of frequency-angle signal parameters. Research showed that (1) frequency-angle selectivity essentially depends on parameters of motion. Common estimates may be essentially better for systems with complicatedly moving antennas than for systems with static antennas or antennas moving with constant velocity; (2) a constant component of velocity makes the common estimates of frequency-angle signal parameters worse and variable components make them better; (3) angle selectivity is mainly determined not by size of array, as for static antenna, but by sizes of area where there is antenna motion (especially periodical motion); (4) in conditions of complicated antenna motion, increasing of angle selectivity takes place even if frequencies of the signal or Doppler's shift are unknown; (5) by correctly taking into consideration complicated antenna motion, there is often a real possibility to increase common frequency-angle selectivity of the systems.

\section{$5: 40$}

4pSP11. Estimation of time-varying signal parameters via VFM Kalman filtering. Sang-Wook Lee (School of Elec. Eng., Seoul Natl. Univ., Seoul 151-742, Korea, 1sw@acoustics.snu.ac.kr), Jun-Seok Lim (Sejong Univ.), Byung-Doo Jun, and Koeng-Mo Sung (Seoul Natl. Univ., Seoul 151-742, Korea)

This paper proposes a VFM (variable fading memory) Kalman filtering and applies it to the parameter estimation for time-varying signal. By adaptively calculating fading memory, the proposed algorithm does not require any fixed predetermined fading memory when estimating a time- 
varying signal. The time-varying signal parameter estimation performance is evaluated by computer simulations for two cases, one of which is the chirp signal whose frequency varies linearly with time and the other is the chirp signal with an impulsive outlier.

\section{6:00}

4pSP12. Coupling criteria for arrays in a channel. Charles F. Gaumond (Acoust. Div., Naval Res. Lab., 4555 Overlook Ave. SW, Washington, DC 20375)

In this paper, an analogy is made and developed between a linear array of receivers with far-field sources in an unbounded, homogeneous medium and an arbitrary array of receivers with arbitrarily placed sources in a channel. The transfer function of a signal transmitted through a channel is the well-studied transmission loss (TL). The singular value decomposition (SVD) is performed on the transfer function between sources and receivers. The three components of the SVD are related to various factors in the case of the linear array in an unbounded, homogeneous medium. The case of a translationally invariant channel described using modes is considered. This modal description is also related to the three components of the SVD. The sum of the eigenvalues of the SVD is proposed as a single number which quantitatively describes the amount of coupling between an arbitrary array and the sound channel, assuming that the distribution of sources spans the propagating modes. This single number can then be used to assess the ability of various array configurations to sample the sound channel. [Work supported by ONR.]

THURSDAY AFTERNOON, 18 MARCH 1999

ROOM H2053, 2:00 TO 6:20 P.M.

\title{
Session 4pUW
}

\section{Underwater Acoustics: Shallow Water}

\author{
Alexandra Tolstoy, Cochair \\ Integrated Performance Decisions, Inc., 8610 Battailles Court, Annandale, Virginia 22003, USA \\ Finn B. Jensen, Cochair \\ SACLANT Undersea Research Centre, Viale San Bartolomeo 400, 19138 La Spezia, Italy
}

\section{Contributed Papers}

\section{2:00}

4pUW1. High-resolution modal mapping in a complex shallow-water environment. Kyle M. Becker (MIT/WHOI Joint Prog. in Oceanogr. and Oceanogr. Eng., Woods Hole Oceanogr. Inst., Woods Hole, MA 02543) and George V. Frisk (Woods Hole Oceanogr. Inst., Woods Hole, MA 02543)

During March 1997, a modal mapping experiment (MOMAX) was conducted to measure the spatial variability of low-frequency sound propagation in shallow water [G. V. Frisk et al., J. Acoust. Soc. Am. 103, 3028(A) (1998)]. The fields created by cw sources in the frequency range $50-300 \mathrm{~Hz}$ were measured using an array of freely drifting hydrophone buoys equipped with GPS navigation. The resulting data consist of complex pressure mapped onto a high-resolution geospatial grid. Based on the tenets of normal mode theory, the wave number content of the field was extracted using a high-resolution implementation of the Hankel tranform technique [K. M. Becker et al., J. Acoust. Soc. Am. 103, 3029(A) (1998)]. Neglecting horizontal refraction, the resulting spectra can be mapped onto a corresponding spatial grid. Several modal maps are presented for various source/receiver configurations and frequencies with emphasis placed on the variablility of the wave numbers in space. These modal maps can then be used as the basis for inferring the local geoacoustic properties of spatially varying shallow-water waveguides. [Work supported by ONR.]

\section{2:20}

4pUW2. Broadband inversion of shallow-water range-dependent acoustic data using a genetic algorithm. Dick G. Simons and Mirjam Snellen (TNO Phys. and Electron Lab., Underwater Acoust. Group, Oude Waalsdorperweg 63, 2509 JG The Hague, The Netherlands)

In October 1997, shallow-water experiments were carried out on the Bank of Adventure southwest of Sicily. The purpose of this trial was to obtain acoustic data in range-dependent environments for testing and further developing matched-field inversion methods to determine geoacoustic properties of the sea floor. On one track of the experiments, an acoustic source was towed over a sloping bottom with a water depth varying from
$100 \mathrm{~m}$ at the vertical receiving array to $75 \mathrm{~m}$ at the end of the $7-\mathrm{km}$ track. The array comprises 64 hydrophones and covered the central $62 \mathrm{~m}$ of the water column. At several source-receiver ranges, the source (towed below the thermocline) transmitted a multitone signal in the band $50-300 \mathrm{~Hz}$. Detailed sound-speed profiling of the water column and seismic profiling of the bottom were performed along the track. Broadband inversion of the acoustic data was done using a genetic algorithm as the global search method. The forward model uses standard adiabatic mode theory. Inversion results are shown for different geoacoustic models with different complexity concerning sediment layering. The bottom parameters obtained agree well with the results of the seismic analysis and its geological interpretation.

\section{2:40}

4pUW3. Analysis of modal dispersion in the Middle Atlantic Bight. John E. Laliberte, Gopu R. Potty, and James H. Miller (Dept. of Ocean Eng., Univ. of Rhode Island, Narragansett, RI 02882)

Ocean environmental acoustic experiments were conducted on the Middle Atlantic Bight during the August 1996 Shelf Break Primer Experiment. Broadband acoustic sources, SUS charges, were dropped across and along the shelf in depths ranging from 85 to $350 \mathrm{~m}$. The experimental data were collected at two veritical hydrophone arrays situated at the top of the shelf. The acoustic signal received was analyzed using a Morlet wavelet. This wavelet analysis enabled time and frequency characteristics to be extracted. Scalograms, representations of the energy distribution in the time-frequency plane, were used to assess the modal dispersion. The group speeds for modes $1-4$ at frequencies $15-240 \mathrm{~Hz}$ were estimated using the wavelet scalogram. The mode dispersion was dependent upon the bathymetry, depth, and range features of the shelf. A normal mode code was used to compare the observed dispersions with theory. In addition, the influence of the bathymetry, depth, and range features were analyzed for their impact on the modal dispersion. [Work supported by ONR.] 
include time-evolving modal power and scintillation indices, and can be interpreted in terms of mode coupling. The ability to locate a source using

4pUW4. Effects of the time-varying ocean on broadband propagation in shallow water. Peter L. Nielsen, Francesco Bini-Verona, and Finn B. Jensen (SACLANTCEN, Viale S. Bartolomeo 400, 19138 La Spezia, Italy, nielsen@saclantc.nato.int)

Prediction of underwater sound propagation is generally performed by assuming a time-invariant ocean. In May 1997 SACLANTCEN conducted an experiment south of Elba in the Mediterranean Sea with the aim of investigating the time-dependent effects of the ocean on sound propagation in shallow water. Broadband LFM signals $(300 \mathrm{~Hz}$ to $7.5 \mathrm{kHz}$ ) were transmitted every $1 \mathrm{~min}$ for $36 \mathrm{~h}$ over a fixed $15-\mathrm{km}$ propagation path. Extensive measurements of the environmental parameters were done during the transmissions in order to perform a meaningful correlation between the time-varying environmental and acoustic data. Previous analysis of the low-frequency part of the acoustic data [Jensen et al., J. Acoust. Soc. Am. 103, 2856(A) (1998)] show high stability in the first $6 \mathrm{~h}$ of propagation, but at longer transmitting time and higher frequencies a strong variability in the received acoustic signals is seen. The main features in the timevarying environmental parameters causing fluctuations in the acoustic signals are identified. These features are then used in a numerical propagation model in order to assess to what degree it is possible to predict the time dependence of the received acoustic data in this particular shallow-water area. [This work was supported by the EC under the MAST-III project PROSIM, Contract No. MAS3-CT95-0008.]

\section{3:20}

4pUW5. The influence of internal waves on signal fluctuation in the Yellow Sea. Zhenge Sun, Renhe Zhang, and Jin Yan (Natl. Lab. of Acoust., Chinese Acad. of Sci., P.O. Box 2712, Beijing, 100080, PROC, szg@farad.ioa.ac.cn)

This paper studies the signal fluctuation due to the tide and internal waves in the Yellow Sea. The spread of internal waves appears to be the fluctuation of the thermocline in summer time. As a result, the characteristics of normal modes including their wave numbers and group velocities vary with this fluctuation. This leads to the signal fluctuations since the receiving signals are actually the interference results of arriving modes. The influence of internal waves on normal modes and signal fluctuation was examined in an experiment in the Yellow Sea in 1998. In the experiment, an ADCP and thermistor chain were used to observe the internal waves, while a vertical array with 32 hydrophones was used to receive $\mathrm{CW}$ and FM acoustic signals. The $\mathrm{CW}$ signals provided the measurement of amplitude fluctuation at several frequencies from 450 to $650 \mathrm{~Hz}$. The FM signals were used to get the mode arriving structure by means of pulse compression and mode filtering technique. The variation of mode group delay between the $3800-\mathrm{Hz}$ signal and the $450-650 \mathrm{~Hz}$ signal was also measured.

\section{3:40}

4pUW6. Acoustic intensity and phase variability caused by timeevolving internal wave fields. Steven Finette, Marshall Orr, Altan Turgut, Stephen Wolf, Bruce Pasewark (Acoust. Div., Naval Res. Lab., Washington, DC 20375, finette@wave.nrl.navy.mil), and John Apel (Global Ocean Assoc., Silver Spring, MD 20908)

Relationships between time-evolving internal wave fields in a shallowwater environment and both acoustic intensity and phase variability are explored through a computer simulation model developed from experimental data acquired on the continental shelf off the New Jersey coast [Apel et al., IEEE J. Ocean. Eng. 22, 465-500 (1997)]. From an analysis of SWARM (Shallow Water Acoustics in a Random Media) data, single realizations of the acoustic field are computed at an array located approximately $42 \mathrm{~km}$ from sources emitting at 224 and $400 \mathrm{~Hz}$. Using a wideangle parabolic equation, the acoustic field is computed in 1-min intervals over a 12-h time window during which both spatially diffuse and spatially localized internal wave fields propagate through the water column. Results phase-coherent techniques such as matched field processing will also be addressed. [Work supported by ONR.]

$$
\text { 4:00-4:20 Break }
$$

$$
4: 20
$$

4pUW7. Characterizations of the internal waves and their effects on sound propagation in the central area of the Yellow Sea. Jin Yan, Renhe Zhang, and Zhenge Sun (Natl. Lab. of Acoust., Chinese Acad. of Sci., Beijing 100080, PROC, yanjin68@hotmail.com)

In the late summer of 1996, a joint China-USA experiment was performed in the central area of the Yellow Sea. The analysis of the data from thermistor string and CTD reveals that (1) there was intense internal wave activity in the experimental area, and the isotherm displacement induced by internal waves could be as large as $8 \mathrm{~m}$; (2) the power spectrum of the vertical displacement had a significant peak near the M2 tide frequency, and was approximately proportional to $\omega^{-3 / 2}$; and (3) the internal wave field was dominated by first-order modes. Based on the observed properties of the internal waves, a model of the induced sound-speed distribution is developed, and computer simulations are conducted for $\mathrm{cw}$ and wideband signal propagation by using the PE method. Numerical results are presented for comparison with the experimental data. [Work supported by NNSF of China and ONR of USA.]

\section{4:40}

4pUW8. Temporal coherence of broadband acoustic signals in the presence of internal waves in shallow water. T. C. Yang and $\mathrm{K}$. Yoo (Naval Res. Lab., Washington, DC 20375)

Ocean medium fluctuations reduce the temporal coherence of acoustic signals. At low (e.g., $500 \mathrm{~Hz}$ ) frequencies, temporal coherence limits the time duration of a replica field used for matched-field source localization in a random ocean. At mid (e.g., $10 \mathrm{kHz}$ ) frequencies, temporal coherence of the acoustic signal dictates the update rate for the channel equalizer for a phase coherent communication scheme. In this paper, the temporal coherence of broadband acoustic signals is studied at low- and midfrequencies in a shallow-water environment which includes both the linear and nonlinear (solitary) internal waves. The internal wave model is constructed based on the measured (average) sound-speed profile and soundspeed variance collected during the SWARM-95 experiment. A broadband pseudorandom (PRN) signal is used at low- and midfrequencies. The signals are propagated using a PE model through the internal wave perturbed sound-speed profiles which evolve in time according to the internal wave equation of motion. Temporal coherence at $400 \mathrm{~Hz}$ is shown to agree with experimental observations based on the SWARM-95 measurements. Implications for midfrequency communications will be discussed. [This work is supported by the Office of Naval Research.]

\section{5:00}

4pUW9. A look at the modal phase structure function in shallow water. Brian J. Sperry (MIT/WHOI Joint Prog. in Oceanogr. and Ocean Eng., Woods Hole Oceanogr. Inst., Woods Hole, MA 02543) and Jim Lynch (Woods Hole Oceanogr. Inst., Woods Hole, MA 02543)

Experimental and theoretical work in shallow-water acoustic propagation has invariably encountered the complexities of the shallow-water environment. A useful measure of the propagation complexity for array processing is the "modal phase structure function." The modal phase structure function makes it possible to study how environmental fluctuations affect point-to-point phase differences, quantities which are directly related to modal coherences. A method has been developed, using perturbation theory, that directly relates the modal phase structure function to the environmental sound-speed correlation function. Despite its relative 
simplicity, this method can address fully coupled mode range dependence, as well as anisotropy in the environmental correlation function. The phase structure function may be applied to time and frequency coherence problems as well. Another strength of the technique is that contributions from various processes, such as mesoscale and finescale variability, interface roughness and geoacoustic variability, may be separated out under reasonable assumptions. Early calculations show good agreement with experimental results. [Work supported by ONR.]

\section{$5: 20$}

4pUW10. Sound vertical coherence in shallow water with a thermocline. Renhe Zhang and Fenghua Li (Natl. Lab. of Acoust., Inst. of Acoust., Chinese Acad. of Sci., Beijing 100 080, PROC zrh@canna.ioa.ac.cn)

A China-U.S. cooperative experiment was conducted in the Far Yellow Sea with a thermocline in August 1996. The explosive sources were discharged either above the thermocline or below the thermocline. The pulsed signals were received by a vertical array spanning the whole water column, and the shortest separation between two hydrophones is 2 meters. The vertical correlation of the received data is analyzed in this paper. Experimental data show that vertical coherence has different depth structure for different source, frequency, and range, and it is also shown that the relationships between the coherence and the frequency and between the coherence and the range are different for different source and receiver. It is found in the experimental data that the vertical coherence is not reciprocal. Numerical results and theoretical explanations of these relationships are also given in this paper. [Work supported by NSF of China and ONR of the U.S.]

\section{$5: 40$}

4pUW11. Mode diffusion and pulse broadening in shallow-water acoustic propagation. Robert I. Odom (Appl. Phys. Lab., Univ. of Washington, 1013 NE 40th St., Seattle, WA 98105)

An acoustic signal propagating in a range-dependent shallow-water waveguide is scattered and attenuated as a result of interaction with medium heterogeneities. An initial signal pulse consisting of a few modes will spread in time linearly with propagation distance as energy is redis- tributed among available modes due to mode coupling. Over longer propagation distances in a random waveguide, modal energy reaches equilibrium, and the average modal energy becomes constant. (The modes are, of course, subject to geometric spreading, intrinsic attenuation, and radiation losses, but it is assumed that all modes are equally affected so that, locally, the energy per mode is a constant.) Energy propagates at a velocity that is an average of the group velocities for individual modes. The pulse width grows mores slowly now as the square root of the propagation distance, rather than linearly. This transition to square-root broadening in randomly perturbed multimode fiber optic waveguides is well known and observed. Employing a diffusion equation for the modal acoustic energy in a random shallow-water waveguide with an elastic bottom, heterogeneity-induced pulse broadening is investigated for realistic environmental properties. [Work supported by ONR.]

\section{6:00}

4pUW12. Effects of internal waves and bores on acoustic transmissions in the Strait of Gibraltar. Christopher O. Tiemann, Peter F. Worcester, Bruce D. Cornuelle (Scripps Inst. of Oceanogr., Univ. of California at San Diego, La Jolla, CA 92093, ctiemann@ucsd.edu), and Uwe Send (Univ. of Kiel, 24105 Kiel, Germany)

The Strait of Gibraltar Acoustic Monitoring Experiment was conducted in April 1996 to determine the feasibility of using acoustic methods to make routine, rapidly repeated transport measurements in the Strait of Gibraltar, as well as to explore the acoustic scattering caused by the unique internal wave field in the Strait. The acoustic data from highfrequency $(2 \mathrm{kHz})$ reciprocal transmissions across the Strait are unique in that they clearly isolate the acoustic effects of passing internal bores without the added complexity of surface and bottom interactions. Although the acoustic scattering caused by each passing internal bore is different, some common characteristics can be identified. The challenge is to explain these acoustic observations in terms of the physical processes occurring. Acoustic propagation models through range- and time-dependent sound-speed fields representing the Strait and perturbed by internal bore models are being used to understand these observations and to determine to what aspects of the bore the acoustics are most sensitive.

\section{Contributed Posters}

These posters will be on display in the Poster Gallery from Wednesday to Friday, 17-19 March. Authors will be at their posters from 10:00 a.m. to 12:00 noon on Friday, 19 March.

4pUW13. High-frequency acoustic propagation measurements in coastal areas. Joseph L. Lopes, Iris C. Paustian, Kerry W. Commander, and John S. Stroud (Coastal Systems Station, Naval Surface Warfare Ctr., Code R21, 6703 West Hwy. 98, Panama City, FL 32407-7001)

A measurement was conducted in a very shallow-water (VSW)/surf zone (SZ) region to investigate acoustic propagation and coherence in coastal areas. The measurement utilized several acoustic sources and hydrophones. The sources were mounted onto a stationary sonar deployed in the VSW. The sonar tower was equipped with a horizontal pan and vertical tilt motors. The hydrophones were attached to steel pipes that were water-jetted into the sediment such that the hydrophones were located in the SZ near the midpoint of the water column. The acoustic frequencies varied from approximately 30 to $800 \mathrm{kHz}$. Data were acquired by directing the acoustic sources (located in the VSW) toward the free-field hydrophones. The data were analyzed using coherence techniques. Results of this acoustic measurement will be reported.
4pUW14. Normal mode propagating through rapid range-dependent shallow water. Fenghua $\mathrm{Li}$ and Renhe Zhang (Natl. Lab. of Acoust., Inst. of Acoust., Chinese Acad. of Sci., Beijing 100 080, PROC, zrh@canna.ioa.ac.cn)

When the normal modes propagate through the range-independent shallow water, the different normal modes are separated into different wave packets in the time domain because of the different group velocities. If the normal modes propagate through some kinds of shallow water which are rapidly range-dependent due to the bottom or the fluctuation in the water column, etc., coupling between different-order modes will happen. And even the same normal mode at the receiver will be divided into two or more wave packets in the time domain, each packet representing the energy coupled from the different normal mode at the source to that mode at the receiver. Those divisions provide a lot of useful information about the rapid range-dependent environment. The divisions of one normal mode are calculated by a coupled mode code which is developed based on BDRM theory, and are also used to estimate the simulated rapid rangedependent environment in this paper. [Work supported by NSF of China.] 
4pUW15. Analysis of active hydroacoustic system's parameters versus conditions of sound propagation in inland water. Jacek Gawrysiak (Tech. Univ. of Gdansk, Narutowicza str 11/12, 80-952 Gdansk, Poland)

The paper contains the numerical calculations of a dependence between the shape of the sound velocity profiles in inland water and sonar parameters such as: directional pattern, range, signal-to-noise ratio, and so on.

4pUW16. On the horizontal structure of a field in separate modes radiated by a parametric antenna in axially nonuniform acoustic waveguides. Sergei Gurbatov, Sergei Egorychev, Vasily Kurin, Nikolai Pronchatov-Rubtsov, and Leonid Kustov (Dept. of Radiophys., Nizhny Novgorod Univ., 23 Gagarin Ave., Nizhny Novgorod, 603600, Russia, gurb@rf.unn.runnet.ru)

The results of laboratory experiments on propagation of low-frequency acoustic waves produced by a parametric antenna in a few-mode waveguide with a linear depth variation along propagation direction are described. Mode structure of the radiated field and the characteristics of eigenwaves forming an acoustic field in waveguides of different types, which model shelf areas of the world oceans, are obtained. The study of the horizontal structure of separate normal waves was carried out by allocation of mode pulses due to the intermode dispersion. Also, the revealing of mode contents of radiated field was carried out by Fourier analysis of the signal received by evenly moving along the propagation route acoustic probe. The different Doppler frequency shifts of eigenmodes allows the resolution of each normal mode. It is shown experimentally that the azimuth structure of separate modes is transformed in the process of sound propagation to an area of smaller waveguide depths. For a particular mode, the direction at which mode radiation reaches a maximum value changes due to decreases in a waveguide depth when the number of normal waves evidently decreases. Experimental data obtained are confirmed by the results of numerical simulations. [Work supported by RFBR Grant No. 98-02-16402 and INTAS-RFBR Grant No. 95-IN-RU-0723.]

4pUW17. Effect of seismic waves on the acoustic field interference structure in the shallow sea. Elena Borodina (Inst. of Appl. Phys., 46 Ulyanov Str., Nizhny Novgorod, 603600, Russia), Sergei Egorychev, Vasily Kurin, Nikolai Pronchatov-Rubtsov (Nizhny Novgorod Univ., Nizhny Novgorod, 603600, Russia, nikvas@ rf.unn.runnet.ru), and Yury Petukhov (Radiophysical Res. Inst., Nizhny Novgorod, 603600, Russia)

The influence of lateral waves on forming of the acoustic field spacefrequency interference structure in a shallow sea was investigated theoretically and experimentally. Within the scope of the simple model of shallow sea as a fluid layer of thickness $H$ on an elastic half-space, the analytical frequency and horizontal range dependencies of amplitudes of compressional and shear lateral waves were derived. Numerical simulation was carried out for natural and laboratory experiment conditions. The numerical results showed the resonant character of frequency dependencies of compressional and shear wave amplitudes, with abrupt narrow maximums at critical frequencies of leak and propagating modes, respectively. It was shown also, that below the critical frequency of the first mode the interference structure is formed mainly by the fundamental mode and the shear wave, which substantially exceeds the compressional one; besides, the shear wave amplitude is almost two times less than the zero mode at distances of about $10 \mathrm{H}$. Thus it was proved that at ranges up to $200 \mathrm{H}$ realized at both natural and laboratory experiment conditions, only the shear wave significantly influences forming of the space-frequency interference field structure in a shallow sea. [Work supported by RFBR Grant No. 98-02-16402, CCPE Grant No. 95-0-8.1-34.] 